\title{
Anatomy of heavy gauge bosons in a left-right supersymmetric model
}

\author{
Biplob Bhattacherjee, ${ }^{1, *}$ Najimuddin Khan, ${ }^{1, \dagger}$ and Ayon Patra ${ }^{2, *}$ \\ ${ }^{1}$ Centre for High Energy Physics, Indian Institute of Science, Bengaluru 560012, India \\ ${ }^{2}$ Physical Research Laboratory, Navrangpura, Ahmedabad 380009, India
}

(Received 8 April 2019; published 10 October 2019)

\begin{abstract}
We perform a detailed study of the various decay channels of the heavy charged and neutral gauge bosons ( $W_{R}$ and $Z_{R}$, respectively) in a left-right supersymmetric framework. The decay branching ratios of the $W_{R}$ and $Z_{R}$ bosons depend significantly on the particle spectrum and composition of the supersymmetric (SUSY) states. We consider several combinations of mass spectrum for the SUSY particles to facilitate the decay of theses heavy gauge bosons into various combinations of final states. Finally, we choose two benchmark points and perform detailed collider simulations for these heavy gauge bosons in the context of the high energy and high luminosity run of the Large Hadron Collider. We analyze two SUSY cascade decay channels, mono- $W+\mathbb{E}_{T}$ and mono- $Z+\mathbb{E}_{T}$, along with the standard dilepton and dijet final states. Our results show that the existence of these heavy gauge bosons can be ascertained in the direct decay channels of dilepton and dijet, whereas the other two channels are required to establish the supersymmetric nature of this model.
\end{abstract}

DOI: $10.1103 /$ PhysRevD.100.075010

\section{INTRODUCTION}

The recent discovery of the Higgs-like scalar boson at the Large Hadron Collider (LHC) [1] has essentially completed the particle spectrum of the standard model (SM). The measured properties of this Higgs-like scalar are consistent with the minimal choice of the scalar sector, as in the SM, with small uncertainties while they still comfortably allow for an extended scalar sector. The Higgs boson, through the spontaneous symmetry breaking mechanism, is responsible for generating masses for the charged fermions, the $W$ and $Z$ bosons, and the Higgs boson itself. ${ }^{1}$ The Higgs boson mass, though, is a very finely tuned parameter in the SM framework and one needs to further extend the SM with new particles or additional symmetries in order to understand the large cancellations required for the observed Higgs boson mass. There are also a number of other experimental observations that lead us to believe that the SM is only an effective low energy theory with new physics coming in at higher energies. Numerous new physics

\footnotetext{
*biplob@iisc.ac.in

†najimuddink@iisc.ac.in

\#ayon@okstate.edu

${ }^{1}$ Neutrinos are massless in the SM framework. The generation of neutrino masses requires an extended framework beyond the SM (BSM) and is an important motivation for BSM scenarios.

Published by the American Physical Society under the terms of the Creative Commons Attribution 4.0 International license. Further distribution of this work must maintain attribution to the author(s) and the published article's title, journal citation, and DOI. Funded by SCOAP ${ }^{3}$.
}

models have thus been suggested to address the shortcomings of the SM.

Left-right supersymmetric (LRSUSY) models [2] are a class of well-motivated extensions of the SM as they can provide answers to a number of unresolved issues in the SM. These are actually the supersymmetric (SUSY) versions of the left-right symmetric (LRS) models [3], where the SM gauge group is extended to $G_{3221}=S U(3)_{c} \times$ $S U(2)_{L} \times S U(2)_{R} \times U(1)_{B-L}$. This extended gauge symmetry facilitates the preservation of parity symmetry at high scales. The observed parity asymmetry in the SM is generated as the LR symmetry is spontaneously broken at some scale $v_{R}$ much above the electroweak (EW) symmetry breaking scale. Parity being a good symmetry in these models can potentially solve the strong $C P$ problem [4] without introducing an additional PecceiQuinn symmetry [5]. The gauge structure in LR models also naturally requires the presence of right-handed neutrinos that can help generate light neutrino masses through the seesaw mechanism [6]. SUSY models, on the other hand, provide an elegant solution to the hierarchy problem. On top of that, if R parity is conserved, as is the case in this paper, the lightest SUSY particle (LSP) becomes stable and can be a good dark matter (DM) candidate. Combining the merits of both LRS and SUSY models, one gets a very attractive LRSUSY framework that warrants a careful examination as is discussed in detail in this paper.

A variety of LRSUSY models have been discussed in literature with different scalar sectors for the symmetry breaking mechanism $[7,8]$. The one we consider here is the minimal LRSUSY model with automatic R-parity 
conservation [9] where the right-handed symmetry is broken by scalar triplet fields as they acquire nonzero vacuum expectation values (VEVs). This VEV is also responsible for generating the Majorana masses for the right-handed neutrinos that eventually help generate light neutrino masses. The spontaneous breaking of the righthanded symmetry gives rise to an additional charged gauge boson $W_{R}$ and a neutral gauge boson $Z_{R}$ whose masses are also at the same scale. The discovery of these gauge bosons could be one of the strongest indication towards the existence of left-right symmetry. Experimental searches for these heavy gauge bosons have been performed by the ATLAS and CMS collaborations in various final states with leptons, jets, and/or missing transverse energy $\left(\mathbb{E}_{T}\right)$, which have helped put bounds on their masses. The CMS search using $36 \mathrm{fb}^{-1}$ of proton-proton collision data collected at a center-of-mass (c.m.) energy of $13 \mathrm{TeV}$ sets the exclusion limit for a heavy neutral gauge boson mass $M_{Z^{\prime}}>4.5 \mathrm{TeV}$ [10] in the dilepton channel for the sequential standard model (SSM). The ATLAS search puts stronger exclusion limit $M_{Z^{\prime}}>5.1 \mathrm{TeV}$ for $139 \mathrm{fb}^{-1}$ in the same dilepton channel [11,12]. Searches at the ATLAS and CMS for heavy charged gauge boson $W^{\prime}$, decaying to $\tau \nu$ (with hadronic decay of $\tau$ ), set lower bound as $M_{W^{\prime}} \gtrsim 4 \mathrm{TeV}$ $[13,14]$. The most stringent mass limit on a heavy charged gauge boson $\left(W^{\prime}\right)$, on the other hand, comes from the CMS collaboration for an integrated luminosity of $35.9 \mathrm{fb}^{-1}$ at $\sqrt{s}=13 \mathrm{TeV}$ energy and is given as $M_{W^{\prime}}>5.2 \mathrm{TeV}$ $[15,16]$ in the lepton $+\mathscr{E}_{T}$ final state for the SSM. This limit, however, does not hold for our analysis as we have chosen the masses of the right-handed neutrinos to be heavier than the $W_{R}$ boson. Thus the lepton $+\mathbb{E}_{T}$ cross section in the final state for $W_{R}$ decay is extremely small in our case, resulting in no significant bound from this channel. Another recent analysis from the ATLAS collaboration [17] gives a mass bound of $M_{W^{\prime}}>3.6 \mathrm{TeV}$ using $37 \mathrm{fb}^{-1}$ integrated luminosity at $13 \mathrm{TeV}$ CoM energy in the dijet $\left(q q^{\prime}\right)$ final state. The mass limit of $3.6 \mathrm{TeV}$ was obtained by assuming a $W^{\prime} \rightarrow q q^{\prime}$ branching ratio (BR) of $75 \%$. For our analyses, on the other hand, this BR could vary from around $50 \%-90 \%$ for different benchmark points (BPs), which significantly affects the results. The heavy right-handed $W_{R}$ bosons decaying into top and a bottom quark searches at $\sqrt{s}=13 \mathrm{TeV}$ by the CMS collaboration also provide a mass limit of $M_{W_{R}}>2.6 \mathrm{TeV}$ [18]. This limit however is much weaker compared to the dijet decay channel. On top of this, all these experimental searches have been performed for the charged and neutral heavy gauge bosons decaying directly into SM particles. For LRSUSY models this is not true at all, since these particles can also decay into SUSY particles, which eventually decay into SM particles and the LSP giving rise to completely new signals.

Phenomenological studies of heavy gauge bosons and their possible discovery channels have also been discussed in several papers. Analysis of the SUSY decays of a heavy $Z^{\prime}$ boson was discussed in Ref. [19], wherein the partial decay widths into all SM and minimal supersymmetric standard model (MSSM) channels were computed and studied in the context of CDF and D0 experiments for a few grand unified theory (GUT)-inspired $U(1)^{\prime}$ scenarios. There have been studies on how the presence of exotic particles and their superpartners could affect the mass limit for the neutral gauge boson in generic $E_{6}$ models [20]. The discovery and measurement of the properties of sleptons, binos, and winos through the production and decay of heavy $Z^{\prime}$ boson into these particles in the extended MSSM with an additional $U(1)$ gauge symmetry has been studied in Ref. [21]. Multilepton $(n=2,4)$ final state searches through $p p \rightarrow Z^{\prime} \rightarrow x x$, where $x=\tilde{l}, \tilde{\chi}_{2}^{ \pm}, \tilde{\nu}$ etc., in the context of LHC were carried out for the GUT-inspired models in [22-25]. The stability analysis of the electroweak potential and corresponding bounds in LRSUSY were studied in Ref. [26]. The CMS eejj excess was explained in the LRSUSY framework $[27,28]$ via the decay of a heavy charged gauge boson.

In this paper, we perform a detailed study of the heavy charged and neutral gauge bosons in a LRSUSY framework with several decay channels in the context of present and future collider experiments. The choice of the right-handed neutrino masses being larger than the heavy gauge bosons significantly changes the allowed decay channels for these particles. We observe that the decay $\mathrm{BR}$ of the $W_{R}$ and $Z_{R}$ bosons depends significantly on the particle spectrum and composition of the SUSY particles (mainly charginos and neutralinos). We thus choose our BPs to encompass all possible compositions for charginos and neutralinos with and without mixing among the fields in the gauge basis. Several combinations of mass spectrum for the SUSY particles are chosen to facilitate the decay of the heavy gauge bosons into various combinations of final states for a more comprehensive study of their properties. This gives us a good understanding of each interaction and how it affects the final decay BR of the heavy gauge bosons. First we consider an almost pure one component LSP, keeping all the squarks and sleptons heavier than both the $W_{R}$ and $Z_{R}$ bosons, such that the heavy gauge bosons cannot decay into final state squarks and sleptons. This allows us to explore their SUSY decays with final states consisting of only charginos and neutralinos. Next we consider the case where the LSP is composed of a significantly mixed state of gauginos and Higgsinos. Finally we allow the squarks and sleptons to be light as well, maximizing the SUSY decay BR for the heavy gauge bosons. Several new decay channels are opened up in some of these cases, which provide completely new collider prospects to search these heavy gauge bosons. These new decay channels can also alter the heavy gauge boson mass limits and allow for new possibilities to discover SUSY particles at the LHC. They have not been discussed previously in the literature, which motivates us to analyze 
this model in the context of high luminosity and high energy LHC experiments.

The discovery prospect of SUSY particles at the LHC is severely constrained by the direct production cross section of these particles. The production cross section falls rapidly as the mass of the particles increases, and hence this translates into upper limits on the masses of SUSY particles that may be discovered at the LHC. The presence of heavy gauge bosons can help mitigate this problem as they can decay into final states with SUSY particles that would otherwise evade detection at the LHC. We analyze four different final states in the context of high luminosity LHC (HL-LHC) and high energy LHC (HE-LHC) experiments. Two of these channels are the standard search channels used for heavy resonance searches-dilepton $+\mathbb{E}_{T}$ final state and dijet $+\mathscr{E}_{T}$ final state. These channels can have large significances in specific benchmark regions in the context of the LHC at 14 and/or $27 \mathrm{TeV}$ energies with integrated luminosity of $3000 \mathrm{fb}^{-1}$. The other two final states arise from the cascade SUSY decays of the heavy gauge bosons resulting in final states with mono $W+\mathbb{E}_{T}$ and mono $Z+\mathbb{E}_{T}$. These signals have already been studied at the LHC for DM searches to constrain them but they have so far not been considered as a search channel for heavy gauge bosons. Our analysis of these final states though gives promising results as a discovery channel for a $W_{R}$ or $Z_{R}$ boson in the LRSUSY framework. We find a large number of events with significance greater than $5 \sigma$ in these new mono- $X(X=W, Z)$ plus $\mathscr{E}_{T}$ channels through one-step cascade decays, with $X$ decaying into leptonic final states only.

The rest of the paper is organized as follows. In Sec. II we present the details of the model and derive expressions for the masses and relevant interactions for all the particles. Section III gives all the different cases that are important for the heavy gauge bosons decays. Here we consider various possible mixings in the neutralino and chargino sectors that affect the $W_{R}$ and $Z_{R}$ BR. We also change the masses of the squarks and sleptons to be heavier or lighter than the gauge bosons so as to study the variation of their decay BRs in each case. The possible collider signals arising from the SUSY decays of the heavy gauge bosons are analyzed in Sec. IV. Here we first study the familiar dilepton and dijet final states for the heavy gauge boson decays. We then analyze a mono $W+\mathbb{E}_{T}$ and a mono $Z+\mathscr{E}_{T}$ final state and how they can be used to search for these heavy particles. Finally we conclude in Sec. V with a discussion of our results. All the relevant interactions of the $W_{R}$ and $Z_{R}$ bosons leading to their decays are provided in the appendixes.

\section{MINIMAL LRSUSY MODEL WITH AUTOMATIC R PARITY}

Left-right symmetric models have an extended gauge symmetry that is $S U(3)_{C} \times S U(2)_{L} \times S U(2)_{R} \times U(1)_{B-L}$.
The chiral fermion sector consists of three families of quark and lepton superfields given as

$$
\begin{aligned}
Q & =\left(\begin{array}{c}
u \\
d
\end{array}\right) \sim\left(3,2,1, \frac{1}{3}\right), \\
Q^{c} & =\left(\begin{array}{c}
d^{c} \\
-u^{c}
\end{array}\right) \sim\left(3^{*}, 1,2,-\frac{1}{3}\right), \\
L & =\left(\begin{array}{c}
\nu \\
e
\end{array}\right) \sim(1,2,1,-1), \\
L^{c} & =\left(\begin{array}{c}
e^{c} \\
-\nu^{c}
\end{array}\right) \sim(1,1,2,1),
\end{aligned}
$$

where $c$ stands for the charge conjugation and the numbers in brackets are their $S U(3)_{C}, S U(2)_{L}, S U(2)_{R}, U(1)_{B-L}$ gauge quantum numbers, respectively.

The minimal Higgs sector required for a consistent symmetry breaking mechanism, generation of quark and lepton masses, and mixings and preservation of an unbroken $R$-parity symmetry is given as

$$
\begin{aligned}
\Delta(1,3,1,2) & =\left(\begin{array}{cc}
\frac{\delta^{+}}{\sqrt{2}} & \delta^{++} \\
\delta^{0} & -\frac{\delta^{+}}{\sqrt{2}}
\end{array}\right), \\
\bar{\Delta}(1,3,1,-2) & =\left(\begin{array}{cc}
\frac{\bar{\delta}^{-}}{\sqrt{2}} & \bar{\delta}^{0} \\
\bar{\delta}^{--} & -\frac{\bar{\delta}^{-}}{\sqrt{2}}
\end{array}\right), \\
\Delta^{c}(1,1,3,-2) & =\left(\begin{array}{cc}
\frac{\delta^{c^{-}}}{\sqrt{2}} & \delta^{c^{0}} \\
\delta^{c^{--}} & -\frac{\delta^{c^{-}}}{\sqrt{2}}
\end{array}\right), \\
\bar{\Delta}^{c}(1,1,3,2) & =\left(\begin{array}{cc}
\frac{\bar{\delta}^{c^{+}}}{\sqrt{2}} & \bar{\delta}^{c^{++}} \\
\bar{\delta}^{c^{0}} & -\frac{\bar{\delta}^{c^{+}}}{\sqrt{2}}
\end{array}\right), \\
\Phi_{i}(1,2,2,0) & =\left(\begin{array}{ll}
\phi_{1}^{+} & \phi_{2}^{0} \\
\phi_{1}^{0} & \phi_{2}^{-}
\end{array}\right)(i=1,2), \quad S(1,1,1,0) .
\end{aligned}
$$

The $S U(2)_{R}$ triplet Higgs field $\Delta^{c}(1,1,3,-2)$ is responsible for breaking the $S U(2)_{R} \times U(1)_{B-L}$ symmetry into $U(1)_{Y}$ as its neutral component acquires a nonzero VEV. The coupling of this triplet field with the right-handed neutrinos generates their Majorana masses as well. For a SUSY model, an extra $S U(2)_{R}$ triplet field $\bar{\Delta}^{c}(1,1,3,+2)$ is also required for anomaly cancellation, and two $S U(2)_{L}$ triplet fields $\Delta(1,3,1,2)$ and $\bar{\Delta}(1,3,1,-2)$ are needed for parity conservation. Two bidoublet fields $\Phi_{a}(1,2,2,0)$ are required to generate the quark and lepton masses and mixings through Yukawa interactions. The simpler case of one bidoublet field, as is considered in our analysis, cannot 
produce the Cabibbo-Kobayashi-Maskawa (CKM) mixing angles. In such a scenario, the CKM mixing angles could arise from soft SUSY breaking terms as discussed in Ref. [29]. The authors in Ref. [29] have chosen their parameters with squark masses of $700 \mathrm{GeV}$ and significantly lighter gluinos, but this choice is ruled out from current experimental bounds [30,31]. It can however be seen from their analysis that we can still obtain the required CKM mixing angles with much heavier squarks and gluino masses by choosing appropriate values for the soft SUSY breaking parameters, the $\mu$ parameter and $\tan \beta$.

The singlet field $S(1,1,1,0)$ is required to decouple the SUSY breaking scale from the right-handed symmetry breaking scale. In the absence of the singlet, the SUSY breaking scale and the right-handed symmetry breaking scale become equal to each other; hence the singlet $S$ is needed in order to decouple the two scales allowing the right-handed symmetry breaking scale to be higher than the SUSY breaking scale.

The nonzero VEVs of various fields are denoted as

$$
\begin{array}{ll}
\left\langle\delta^{c^{0}}\right\rangle=v_{R}, & \left\langle\bar{\delta}^{c^{0}}\right\rangle=\bar{v}_{R}, \quad\left\langle\phi_{1_{i}}^{0}\right\rangle=v_{u_{i}}, \\
\left\langle\phi_{2_{i}}^{0}\right\rangle=v_{d_{i}}, & \langle S\rangle=v_{s},
\end{array}
$$

with the hierarchy among them chosen as $v_{R}, \bar{v}_{R}>v_{s} \gg$ $v_{u}, v_{d}$. The right-handed symmetry breaking scale is chosen larger than the SUSY breaking scale as we want the superpartner masses to be lighter than the heavy $W_{R}$ and $Z_{R}$ gauge bosons. For simplicity, the left-handed $\delta^{0}$ and $\bar{\delta}^{0}$ fields do not get any VEVs and hence the neutrino masses do not get any contribution from type-II seesaw. This choice is a consistent one as it can also be shown that the left-handed triplet fields do not get any induced VEV in this model.

The Yukawa couplings in the model are given by the superpotential

$$
\begin{aligned}
W_{Y}= & \sum_{j=1}^{2}\left(Y_{q}^{(j)} Q^{T} \tau_{2} \Phi_{j} \tau_{2} Q^{c}+Y_{l}^{(j)} L^{T} \tau_{2} \Phi_{j} \tau_{2} L^{c}\right) \\
& +i \frac{f}{2} L^{T} \tau_{2} \Delta L+i \frac{f^{c}}{2} L^{c T} \tau_{2} \Delta^{c} L^{c} .
\end{aligned}
$$

Here $Y_{l}^{j}$ and $Y_{q}^{j}$ are the lepton and quark Yukawa coupling matrices, respectively, while $f$ is the Majorana Yukawa coupling matrix responsible for generating large Majorana masses for right-handed neutrinos. The transformation of various fields under parity symmetry is given as $\Phi \rightarrow \Phi^{\dagger}, \Delta \rightarrow \Delta^{c^{*}}, \bar{\Delta} \rightarrow \bar{\Delta}^{c^{*}}, S \rightarrow S^{*}, Q \rightarrow Q^{c^{*}}, L \rightarrow L^{c^{*}}$, $\theta \rightarrow \bar{\theta}$, along with $W^{ \pm} \rightarrow W_{R}^{ \pm *}$. Additionally the Yukawa superpotential is invariant under parity if the Yukawa coupling matrices $Y_{q}^{j}$ and $Y_{l}^{j}$ are Hermitian and $f^{c}=f$. The up quarks, down quarks, charged leptons, neutrino Dirac, and right-handed Majorana neutrino masses are given as

$$
\begin{array}{ll}
\boldsymbol{M}_{\boldsymbol{u}}=Y_{q}^{(1)} v_{u_{1}}+Y_{q}^{(2)} v_{u_{2}}, & \boldsymbol{M}_{\boldsymbol{d}}=Y_{q}^{(1)} v_{d_{1}}+Y_{q}^{(2)} v_{d_{2}} \\
\boldsymbol{M}_{\ell}=Y_{l}^{(1)} v_{d_{1}}+Y_{l}^{(2)} v_{d_{2}}, & \boldsymbol{M}_{\boldsymbol{\nu}}^{\boldsymbol{D}}=Y_{l}^{(1)} v_{u_{1}}+Y_{l}^{(2)} v_{u_{2}} \\
\boldsymbol{M}_{\boldsymbol{R}}=f v_{R}, &
\end{array}
$$

respectively. Thus it is easy to see that two bidoublet Higgs fields are needed to generate the CKM mixings as otherwise the up- and down-type quark mass matrices would become proportional to each other at the tree level. As has been discussed earlier, the one loop corrections arising from the soft SUSY breaking terms can help obtain the correct quark masses and generate the CKM mixings even with one bidoublet scalar in this scenario. We thus consider the simplified version of the model with one bidoublet scalar for our analysis.

The gauge sector of the model has an extra charged $W_{R}$ and a neutral $Z_{R}$ gauge boson. The mass-squared matrices for the neutral gauge boson $\boldsymbol{M}_{Z}^{2}$ in the basis $\left(B, W_{3 L}, W_{3 R}\right)$ and the charged gauge boson $\boldsymbol{M}_{W}^{2}$ in the basis $\left(W_{L}, W_{R}\right)$ are given as

$$
\boldsymbol{M}_{\boldsymbol{Z}}^{2}=\frac{1}{2}\left[\begin{array}{ccc}
4 g_{V}^{2}\left(v_{R}^{2}+\bar{v}_{R}^{2}\right) & 0 & -4 g_{R} g_{V}\left(v_{R}^{2}+\bar{v}_{R}^{2}\right) \\
0 & g_{2}^{2} v^{2} & g_{2} g_{R} v^{2} \\
-4 g_{R} g_{V}\left(v_{R}^{2}+\bar{v}_{R}^{2}\right) & g_{2} g_{R} v^{2} & g_{R}^{2}\left(4 v_{R}^{2}+4 \bar{v}_{R}^{2}+v^{2}\right)
\end{array}\right], \quad \boldsymbol{M}_{\boldsymbol{W}}^{2}=\frac{1}{2}\left[\begin{array}{cc}
g_{2}^{2} v^{2} & g_{2} g_{R} v_{1} v_{2} \\
g_{2} g_{R} v_{1} v_{2} & g_{R}^{2}\left(2 v_{R}^{2}+2 \bar{v}_{R}^{2}+v^{2}\right),
\end{array}\right],
$$

where $v^{2}=v_{u}^{2}+v_{d}^{2}=174.1 \mathrm{GeV}$ while $g_{V}, g_{2}$, and $g_{R}$ are the gauge coupling constants corresponding to the $U(1)_{B-L}, S U(2)_{L}$, and $S U(2)_{R}$ gauge groups, respectively. The mass eigenvalues of the heavy gauge bosons can be obtained as

$$
M_{W_{R}}^{2} \simeq \frac{1}{2} g_{R}^{2}\left(2 v_{R}^{2}+2 \bar{v}_{R}^{2}+v^{2}\right), \quad M_{Z_{R}}^{2} \simeq \frac{1}{2}\left(g_{R}^{2}+g_{V}^{2}\right)\left[4\left(v_{R}^{2}+\bar{v}_{R}^{2}\right)+v^{2} \cos ^{4} \theta_{R}\right],
$$


where $\cos ^{2} \theta_{R}=g_{R}^{2} /\left(g_{R}^{2}+g_{V}^{2}\right)$. In getting these masses we have neglected the mixing between the left- and right-handed charged gauge bosons and neglected terms of order $v^{4} / v_{R}^{4}$ or higher. The SM gauge bosons have their usual expressions with the effective $U(1)_{Y}$ hypercharge coupling identified as $g_{Y}^{2}=g_{R}^{2} g_{V}^{2} /\left(g_{R}^{2}+g_{V}^{2}\right)$. The ratio of the heavy gauge boson masses can be approximately written as [2]

$$
\frac{M_{Z_{R}}}{M_{W_{R}}} \simeq \frac{\sqrt{2} g_{R} / g_{2}}{\sqrt{\left(g_{R} / g_{2}\right)^{2}-\tan ^{2} \theta_{W}}},
$$

where $\theta_{W}$ is the Weinberg angle. This relation shows that the ratio $g_{R} / g_{2}$ should always be larger than $\tan \theta_{W}$.

The most general superpotential for the Higgs sector is given as

$$
\begin{aligned}
W= & S\left[\operatorname{Tr}(\lambda \Delta \bar{\Delta})+\operatorname{Tr}\left(\lambda^{c} \Delta^{c} \bar{\Delta}^{c}\right)+\frac{\lambda^{\prime}}{2} \operatorname{Tr}\left(\Phi^{T} \tau_{2} \Phi \tau_{2}\right)-M^{2}\right] \\
& +\operatorname{Tr}\left[\mu_{1} \Delta \bar{\Delta}+\mu_{2} \Delta^{c} \bar{\Delta}^{c}+\frac{\mu}{2}\left(\Phi^{T} \tau_{2} \Phi \tau_{2}\right)\right] \\
& +\frac{\mu_{S}}{2} S^{2}+\frac{\kappa}{3} S^{3}
\end{aligned}
$$

where $\lambda^{c}=\lambda^{*}, \mu_{1}=\mu_{2}^{*}$ while $\lambda_{0}, M_{2}, \mu$ and $\mu_{S}$ are all real from the conservation of parity symmetry. The Higgs potential derived from this superpotential consists of $F$-terms, $D$-terms, and soft supersymmetry breaking terms. So we have

$$
V_{\text {Higgs }}=V_{F}+V_{D}+V_{\text {Soft }} \text {, }
$$

with each of the terms being

$$
\begin{aligned}
V_{F}=\left|\operatorname{Tr}(\lambda \Delta \bar{\Delta})+\operatorname{Tr}\left(\lambda^{c} \Delta^{c} \bar{\Delta}^{c}\right)+\frac{\lambda^{\prime}}{2} \operatorname{Tr}\left(\Phi^{T} \tau_{2} \Phi \tau_{2}\right)-M^{2}+\mu_{S} S+\kappa S^{2}\right|^{2} \\
+\operatorname{Tr}\left[\left|\mu_{1} \Delta+\lambda S \Delta\right|^{2}+\left|\mu_{1} \bar{\Delta}+\lambda S \bar{\Delta}\right|^{2}+\left|\mu_{1}^{*} \Delta^{c}+\lambda^{*} S \Delta^{c}\right|^{2}\right. \\
\left.+\left|\mu_{1}^{*} \bar{\Delta}^{c}+\lambda^{*} S \bar{\Delta}^{c}\right|^{2}\right]+\operatorname{Tr}\left|\mu \Phi+\lambda^{\prime} S \Phi\right|^{2}, \\
V_{D}=\frac{g_{2}^{2}}{8} \sum_{a=1}^{3}\left|\operatorname{Tr}\left(2 \Delta^{\dagger} \tau_{a} \Delta+2 \bar{\Delta}^{\dagger} \tau_{a} \bar{\Delta}+\Phi^{\dagger} \tau_{a} \Phi\right)\right|^{2} \\
+\frac{g_{R}^{2}}{8} \sum_{a=1}^{3}\left|\operatorname{Tr}\left(2 \Delta^{c \dagger} \tau_{a} \Delta^{c}+2 \bar{\Delta}^{c \dagger} \tau_{a} \bar{\Delta}^{c}+\Phi^{*} \tau_{a} \Phi^{T}\right)\right|^{2} \\
\quad+\frac{g_{V}^{2}}{2}\left|\operatorname{Tr}\left(\Delta^{\dagger} \Delta-\bar{\Delta}^{\dagger} \bar{\Delta}-\Delta^{c \dagger} \Delta^{c}+\bar{\Delta}^{c^{\dagger}} \bar{\Delta}^{c}\right)\right|^{2}, \\
V_{\text {Soft }}=m_{1}^{2} \operatorname{Tr}\left(\Delta^{c \dagger} \Delta^{c}\right)+m_{2}^{2} \operatorname{Tr}\left(\bar{\Delta}^{c \dagger} \bar{\Delta}^{c}\right)+m_{3}^{2} \operatorname{Tr}\left(\Delta^{\dagger} \Delta\right)+m_{4}^{2} \operatorname{Tr}\left(\bar{\Delta}^{\dagger} \bar{\Delta}\right) \\
+m_{S}^{2}|S|^{2}+m_{5}^{2} \operatorname{Tr}\left(\Phi^{\dagger} \Phi\right)+\left[\lambda A_{\lambda} S \operatorname{Tr}\left(\Delta \bar{\Delta}+\Delta^{c} \bar{\Delta}^{c}\right)+\text { H.c. }\right] \\
+\left[\lambda^{\prime} A_{\lambda^{\prime}} S \operatorname{Tr}\left(\Phi^{T} \tau_{2} \Phi \tau_{2}\right)+\operatorname{H.c} .\right]+\left(\lambda C_{\lambda} M^{2} S+\text { H.c. }\right)+\left(\mu_{S} B_{S} S^{2}+\text { H.c. }\right) \\
+\left[\mu_{1} B_{1} \operatorname{Tr}(\Delta \bar{\Delta})+\mu_{1}^{*} B_{2} \operatorname{Tr}\left(\Delta^{c} \bar{\Delta}^{c}\right)+\mu B \operatorname{Tr}\left(\Phi^{T} \tau_{2} \Phi \tau_{2}\right)+\kappa A_{\kappa} S^{3}+\text { H.c. }\right] .
\end{aligned}
$$

The minimization of the scalar potential proceeds as

$$
\begin{gathered}
\left|\frac{\partial V_{\text {Higgs }}}{\partial \phi_{i}}\right|_{\phi_{i}=v_{i}}=0, \\
\frac{\partial V}{\partial \phi_{1}^{0}}=\frac{1}{2 \sqrt{2}} v_{u}\left\{g_{2}^{2}\left(-v_{d}^{2}+v_{u}^{2}\right)+g_{R}^{2}\left(2 \bar{v}_{R}^{2}-2 v_{R}^{2}-v_{d}^{2}+v_{u}^{2}\right)\right\}+\sqrt{2}\left\{\lambda^{\prime 2}\left(v_{d}^{2}+v_{s}^{2}\right)+\mu^{2}+m_{5}^{2}\right\} v_{u} \\
-\lambda^{\prime}\left[v_{d}\left(\lambda^{c} v_{R} \bar{v}_{R}-M^{2}\right)+v_{s}\left\{-2 \mu v_{u}+v_{d}\left(\mu_{s}+k v_{s}\right)\right\}\right]-2 v_{d}\left(v_{s} \lambda^{\prime} A_{\lambda^{\prime}}+\mu B\right)=0, \\
\begin{aligned}
\frac{\partial V}{\partial \phi_{2}^{0}}= & \frac{1}{2 \sqrt{2}} v_{d}\left\{g_{2}^{2}\left(v_{d}^{2}-v_{u}^{2}\right)+g_{R}^{2}\left(-2 \bar{v}_{R}^{2}+2 v_{R}^{2}-v_{u}^{2}+v_{d}^{2}\right)\right\}+\sqrt{2} v_{d}\left(\mu^{2}+2 \lambda^{\prime} \mu v_{s}+m_{5}^{2}\right) \\
& -\lambda^{\prime}\left\{v_{u}\left(\mu_{s} v_{s}+k v_{s}^{2}+\lambda^{c} v_{R} \bar{v}_{R}-M^{2}\right)-\lambda^{\prime} v_{d}\left(v_{s}^{2}+v_{u}^{2}\right)\right\}-2 v_{u}\left(v_{s} \lambda^{\prime} A_{\lambda^{\prime}}+\mu B\right)=0,
\end{aligned}
\end{gathered}
$$




$$
\begin{aligned}
\frac{\partial V}{\partial \delta^{c^{0}}}= & \frac{1}{\sqrt{2}} \bar{v}_{R}\left\{2 g_{V}^{2}\left(\bar{v}_{R}^{2}-v_{R}^{2}\right)+g_{R}^{2}\left(2 \bar{v}_{R}^{2}-2 v_{R}^{2}-v_{d}^{2}+v_{u}^{2}\right)\right\}+\sqrt{2} \bar{v}_{R}\left(2 \lambda^{c} \mu_{2} v_{s}+m_{1}^{2}+\mu_{2}^{2}\right) \\
& +\lambda^{c 2} \bar{v}_{R}\left(v_{R}^{2}+v_{s}^{2}\right)+\lambda^{c} v_{R}\left(-\lambda^{\prime} v_{d} v_{u}+\mu_{s} v_{s}+k v_{s}^{2}-M^{2}\right)+v_{R}\left(v_{s} \lambda A_{\lambda}+\mu_{1}^{*} B_{2}\right)=0 \\
\frac{\partial V}{\partial \bar{\delta}^{c^{0}}}= & \frac{1}{\sqrt{2}} v_{R}\left\{2 g_{V}^{2}\left(v_{R}^{2}-\bar{v}_{R}^{2}\right)+g_{R}^{2}\left(-2 \bar{v}_{R}^{2}+2 v_{R}^{2}-v_{u}^{2}+v_{d}^{2}\right)\right\}+\sqrt{2} v_{R}\left(2 \lambda^{c} \mu_{2} v_{s}+m_{2}^{2}+\mu_{2}^{2}\right) \\
& +\lambda^{c} \bar{v}_{R}\left(-\lambda^{\prime} v_{d} v_{u}+\mu_{s} v_{s}+k v_{s}^{2}-M^{2}\right)+\lambda^{c 2} v_{R}\left(\bar{v}_{R}^{2}+v_{s}^{2}\right)+\bar{v}_{R}\left(v_{s} \lambda A_{\lambda}+\mu_{1}^{*} B_{2}\right)=0 \\
\frac{\partial V}{\partial S}= & \sqrt{2}\left\{\lambda^{c} v_{R} \bar{v}_{R}\left(2 k v_{s}+\mu_{s}\right)+\lambda^{c} \mu_{2}\left(v_{R}^{2}+\bar{v}_{R}^{2}\right)+\mu \lambda^{\prime}\left(v_{d}^{2}+v_{u}^{2}\right)-\lambda^{\prime}\left(2 k v_{s}+\mu_{s}\right) v_{d} v_{u}\right\} \\
& -\left(2 k v_{s}+\mu_{s}\right) M^{2}+\lambda C_{\lambda}+v_{R} \bar{v}_{R} \lambda A_{\lambda}+v_{s}\left\{\left(2 k v_{s}+\mu_{s}\right)\left(\mu_{s}+k v_{s}\right)+2 \mu_{s} B_{s}+3 v_{s} k A_{k}\right. \\
& \left.+\lambda^{\prime 2}\left(v_{d}^{2}+v_{u}^{2}\right)+\lambda^{c 2}\left(v_{R}^{2}+\bar{v}_{R}^{2}\right)+m_{S}^{2}-2 v_{d} v_{u} \lambda^{\prime} A_{\lambda^{\prime}}\right\}=0 .
\end{aligned}
$$

\section{A. Particle masses}

In this section we calculate the masses of the particles in various sectors of our LRSUSY model.

\section{Higgs sector}

The mass-squared matrices for the charged and neutral Higgs bosons can be obtained from the scalar potential. The minimization conditions in Eqs. (15)-(19) provide further constraints on the parameters in the model. The singlet $(S)$, the bidoublet $(\Phi)$, and the right-handed triplets $\left(\Delta^{c}\right.$ and $\left.\bar{\Delta}^{c}\right)$ can mix with each other while the left-handed triplets $(\Delta$ and $\bar{\Delta})$ get decoupled since they do not acquire any VEVs. Here we only consider the sector consisting of the right-handed triplets, the bidoublet, and the singlet as these are important for our analysis of the heavy right-handed gauge bosons.

After electroweak symmetry breaking, the mass-squared matrix for the singly charged Higgs fields can be expressed as a $4 \times 4$ matrix in the basis $\left(\delta^{c^{ \pm}}, \bar{\delta}^{c^{ \pm}}, \phi_{2}^{ \pm}, \phi_{1}^{ \pm}\right)$as

$$
\boldsymbol{m}_{\boldsymbol{H} \boldsymbol{m}}^{\mathbf{2}}=\left(\begin{array}{cccc}
m_{\delta^{c^{-}} \delta^{c^{+}}} & m_{\bar{\delta}^{c^{-}} \delta^{c^{+}}}^{*} & -\frac{1}{\sqrt{2}} g_{R}^{2} \bar{v}_{R} v_{u} & -\frac{1}{\sqrt{2}} g_{R}^{2} v_{d} \bar{v}_{R} \\
m_{\delta^{c^{-}} \bar{\delta}^{c^{+}}} & m_{\bar{\delta}^{c^{-}} \bar{\delta}^{c^{+}}} & \frac{1}{\sqrt{2}} g_{R}^{2} v_{R} v_{u} & \frac{1}{\sqrt{2}} g_{R}^{2} v_{d} v_{R} \\
-\frac{1}{\sqrt{2}} g_{R}^{2} \bar{v}_{R} v_{u} & \frac{1}{\sqrt{2}} g_{R}^{2} v_{R} v_{u} & m_{\phi_{2}^{-} \phi_{2}^{+}} & m_{\phi_{1}^{-} \phi_{2}^{+}}^{*} \\
-\frac{1}{\sqrt{2}} g_{R}^{2} v_{d} \bar{v}_{R} & \frac{1}{\sqrt{2}} g_{R}^{2} v_{d} v_{R} & m_{\phi_{2}^{-} \phi_{1}^{+}} & m_{\phi_{1}^{-} \phi_{1}^{+}}
\end{array}\right),
$$

where

$$
\begin{aligned}
& m_{\delta^{c^{-}} \delta^{c^{+}}}=\left(g_{V}^{2}+g_{R}^{2}\right) \bar{v}_{R}^{2}-g_{V}^{2} v_{R}^{2}+m_{1}^{2}+\left(\lambda^{c} v_{s}+\mu_{2}\right)^{2}, \\
& m_{\delta^{c^{-}} \bar{\delta}^{c^{+}}}=-g_{R}^{2} v_{R} \bar{v}_{R}+\lambda^{c}\left(-\lambda^{\prime} v_{d} v_{u}+\mu_{s} v_{s}+k v_{s}^{2}+\lambda^{c} v_{R} \bar{v}_{R}-M^{2}\right)+v_{s} \lambda A_{\lambda}+\mu_{1}^{*} B_{2}, \\
& m_{\bar{\delta}^{c^{-}} \bar{\delta}^{c^{+}}}=g_{V}^{2}\left(-\bar{v}_{R}^{2}+v_{R}^{2}\right)+g_{R}^{2} v_{R}^{2}+m_{2}^{2}+\left(\lambda^{c} v_{s}+\mu_{2}\right)^{2}, \\
& m_{\phi_{2}^{-} \phi_{2}^{+}}=\left(\lambda^{\prime} v_{s}+\mu\right)^{2}+\frac{1}{4}\left\{g_{2}^{2}\left(v_{d}^{2}+v_{u}^{2}\right)+g_{R}^{2}\left(-2 \bar{v}_{R}^{2}+2 v_{R}^{2}+v_{d}^{2}+v_{u}^{2}\right)\right\}+m_{5}^{2}, \\
& m_{\phi_{2}^{-} \phi_{1}^{+}}=\lambda^{\prime}\left(\mu_{s} v_{s}-\lambda^{\prime} v_{d} v_{u}+k v_{s}^{2}+\lambda^{c} v_{R} \bar{v}_{R}-M^{2}\right)+2 v_{s} \lambda^{\prime} A_{\lambda^{\prime}}+2 \mu B+\frac{\left(g_{2}^{2}+g_{R}^{2}\right)}{2} v_{d} v_{u}, \\
& m_{\phi_{1}^{-} \phi_{1}^{+}}=\left(\lambda^{\prime} v_{s}+\mu\right)^{2}+\frac{1}{4}\left\{g_{2}^{2}\left(v_{d}^{2}+v_{u}^{2}\right)+g_{R}^{2}\left(2 \bar{v}_{R}^{2}-2 v_{R}^{2}+v_{d}^{2}+v_{u}^{2}\right)\right\}+m_{5}^{2} .
\end{aligned}
$$

The singly charged fields in the left-handed triplets get decoupled and are not important for our analysis. As a result they have not been included here. The $4 \times 4$ mass-squared matrix in Eq. (20) can be diagonalized by the transformation $U^{H m} \mathbf{m}_{\mathbf{H m}}^{2} U^{H m^{\dagger}}=m_{H m \text {,diag }}^{2}$, where $U^{H m}$ stands for the rotation matrix for the singly charged Higgs fields. This gives two physical mass eigenstates $\left(H_{1}^{ \pm}, H_{2}^{ \pm}\right)$for the charged scalar fields while the remaining two states $\left(G_{1}^{ \pm}, G_{2}^{ \pm}\right)$become the massless Goldstone bosons. These massless degrees of freedom are eaten up by their corresponding gauge bosons $W^{ \pm}$and $W_{R}$, respectively, to give them mass. 
The mass-squared matrix for the doubly charged scalar fields is given by a $2 \times 2$ matrix in the basis $\left(\delta^{c^{ \pm \pm}}, \bar{\delta}^{ \pm \pm \pm}\right)$. This can be written as

$$
\boldsymbol{m}_{\mathbf{H} \boldsymbol{m} \boldsymbol{m}}^{2}=\left(\begin{array}{cc}
m_{\delta^{c^{--}} \delta^{c^{++}}} & m_{\bar{\delta}^{c^{--}} \delta^{c^{++}}}^{*} \\
m_{\delta^{c^{--}} \bar{\delta}^{c^{++}}} & m_{\bar{\delta}^{c^{--}} \bar{\delta}^{c^{++}}}
\end{array}\right),
$$

where

$$
\begin{aligned}
& m_{\delta^{c^{--}} \delta^{c+}}=\frac{g_{R}^{2}}{2}\left(-2 \bar{v}_{R}^{2}+2 v_{R}^{2}-v_{u}^{2}+v_{d}^{2}\right)+g_{V}^{2}\left(\bar{v}_{R}^{2}-v_{R}^{2}\right)+m_{1}^{2}+\left(\lambda^{c} v_{s}+\mu_{2}\right)^{2}, \\
& m_{\delta^{c^{-}} \bar{\delta}^{c^{++}}}=\lambda^{c}\left(\mu_{s} v_{s}-\lambda^{\prime} v_{d} v_{u}+k v_{s}^{2}+\lambda^{c} v_{R} \bar{v}_{R}-M^{2}\right)+v_{s} \lambda A_{\lambda}+\mu_{1}^{*} B_{2}, \\
& m_{\bar{\delta}^{c^{-}} \bar{\delta}^{c^{++}}}=\frac{g_{R}^{2}}{2}\left(2 \bar{v}_{R}^{2}-2 v_{R}^{2}-v_{d}^{2}+v_{u}^{2}\right)+g_{V}^{2}\left(v_{R}^{2}-\bar{v}_{R}^{2}\right)+m_{2}^{2}+\left(\lambda^{c} v_{s}+\mu_{2}\right)^{2} .
\end{aligned}
$$

It can be shown that this doubly charged Higgs mass-squared matrix, upon diagonalization, admits a negative eigenvalue that is unphysical as it gives rise to a tachyonic state. This problem can however be solved by including the radiative corrections to the doubly charged Higgs boson mass that make it positive [8,9]. A numerical analysis of the one-loop corrections to the doubly charged Higgs boson mass in this model has been performed in Ref. [26]. Figure 5 of Ref. [26] shows the different contributions to the doubly charged Higgs boson mass where one can easily see that the contribution from the (s)lepton and (s)neutrino sector (denoted by the dotted blue line in the figure) is always negative in this case. An analytical expression for this contribution has also been derived in Ref. [8], which shows that it can be positive or negative depending on the ratio of the slepton mass to the right-handed neutrino mass. If the right-handed neutrino is heavier than the slepton, the correction is always negative while it can become positive if the slepton mass is slightly larger than (greater than 1.65 times) the right-handed neutrino mass. Thus the one loop corrected doubly charged Higgs boson mass is highly dependent on the chosen mass spectrum for the BSM particles and can be made consistent with the experimental bounds.

The $C P$-even neutral scalars consist of the real part of the neutral Higgs fields. The mass-squared matrix for these fields in the basis $\left(\operatorname{Re}\left[\delta^{c^{0}}\right], \operatorname{Re}\left[\bar{\delta}^{c^{0}}\right], \operatorname{Re}\left[\phi_{2}^{0}\right], \operatorname{Re}\left[\phi_{1}^{0}\right], \operatorname{Re}[S]\right)$ is given as

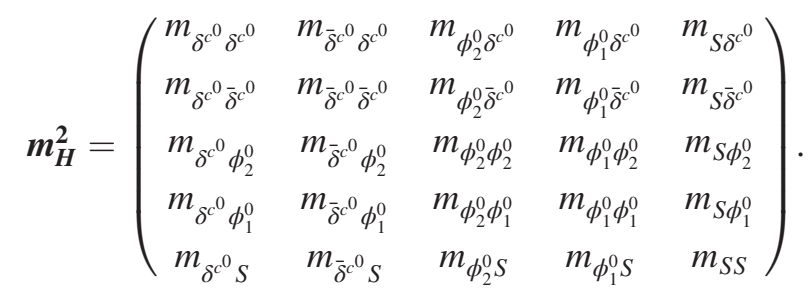

The matrix elements are defined as

$$
\begin{aligned}
& m_{\delta^{c^{0}} \delta^{c^{0}}}=\frac{g_{R}^{2}}{2}\left(6 \bar{v}_{R}^{2}-v_{d}^{2}-2 v_{R}^{2}+v_{u}^{2}\right)-g_{V}^{2}\left(-3 \bar{v}_{R}^{2}+v_{R}^{2}\right)+\lambda^{c 2} v_{R}^{2}+m_{1}^{2}+\left(\lambda^{c} v_{s}+\mu_{2}\right)^{2}, \\
& m_{\delta^{c} \bar{\delta}^{c^{0}}}=\lambda^{c}\left\{\left(2 \lambda^{c} v_{R} \bar{v}_{R}+\mu_{s} v_{s}\right)-\lambda^{\prime} v_{d} v_{u}+k v_{s}^{2}-M^{2}\right\}-2\left(g_{V}^{2}+g_{R}^{2}\right) v_{R} \bar{v}_{R}+v_{s} \lambda A_{\lambda}+\mu_{1}^{*} B_{2}, \\
& m_{\bar{\delta}^{c^{0}} \bar{\delta}^{c^{0}}}=g_{V}^{2}\left(3 v_{R}^{2}-\bar{v}_{R}^{2}\right)+\frac{g_{R}^{2}}{2}\left(-2 \bar{v}_{R}^{2}+6 v_{R}^{2}-v_{u}^{2}+v_{d}^{2}\right)+\lambda^{c 2} \bar{v}_{R}^{2}+m_{2}^{2}+\left(\lambda^{c} v_{s}+\mu_{2}\right)^{2}, \\
& m_{\delta^{c^{0}} \phi_{2}^{0}}=-\lambda^{c} \lambda^{\prime} v_{R} v_{u}-g_{R}^{2} v_{d} \bar{v}_{R}, \quad m_{\bar{\delta}^{c^{0}} \phi_{2}^{0}}=-\lambda^{c} \lambda^{\prime} \bar{v}_{R} v_{u}+g_{R}^{2} v_{d} v_{R}, \\
& m_{\phi_{2}^{0} \phi_{2}^{0}}=\lambda^{\prime 2} v_{u}^{2}+\left(\lambda^{\prime} v_{s}+\mu\right)^{2}+\frac{g_{2}^{2}}{4}\left(3 v_{d}^{2}-v_{u}^{2}\right)+g_{R}^{2}\left(-2 \bar{v}_{R}^{2}+2 v_{R}^{2}+3 v_{d}^{2}-v_{u}^{2}\right)+m_{5}^{2}, \\
& m_{\delta^{c^{0}} \phi_{1}^{0}}=-\lambda^{c} \lambda^{\prime} v_{d} v_{R}+g_{R}^{2} \bar{v}_{R} v_{u}, \quad m_{\bar{\delta}^{0} \phi_{1}^{0}}=-\lambda^{c} \lambda^{\prime} v_{d} \bar{v}_{R}-g_{R}^{2} v_{R} v_{u}, \\
& m_{\phi_{2}^{0} \phi_{1}^{0}}=-\lambda^{\prime}\left(\mu_{s} v_{s}+k v_{s}^{2}-2 \lambda^{\prime} v_{d} v_{u}+\lambda^{c} v_{R} \bar{v}_{R}-M^{2}\right)-2 v_{s} \lambda^{\prime} A_{\lambda^{\prime}}-2 \mu B-\frac{\left(g_{2}^{2}+g_{R}^{2}\right)}{2} v_{d} v_{u}, \\
& m_{\phi_{1}^{0} \phi_{1}^{0}}=\left\{\lambda^{\prime 2} v_{d}^{2}+\left(\lambda^{\prime} v_{s}+\mu\right)^{2}\right\}-\frac{g_{2}^{2}}{4}\left(-3 v_{u}^{2}+v_{d}^{2}\right)-\frac{g_{R}^{2}}{4}\left(-2 \bar{v}_{R}^{2}+2 v_{R}^{2}-3 v_{u}^{2}+v_{d}^{2}\right)+m_{5}^{2},
\end{aligned}
$$




$$
\begin{aligned}
m_{\delta^{0} S}= & 2 \lambda^{c} \bar{v}_{R}\left(\lambda^{c} v_{s}+\mu_{2}\right)+\lambda^{c} v_{R}\left(2 k v_{s}+\mu_{s}\right)+v_{R} \lambda A_{\lambda}, \\
m_{\bar{\delta}^{0} S}= & \lambda^{c} \bar{v}_{R}\left(2 k v_{s}+\mu_{s}\right)+2 \lambda^{c} v_{R}\left(\lambda^{c} v_{s}+\mu_{2}\right)+\bar{v}_{R} \lambda A_{\lambda}, \\
m_{\phi_{2}^{0} S}= & -\lambda^{\prime}\left(2 k v_{s}+\mu_{s}\right) v_{u}+4 \lambda^{\prime} v_{d}\left(\lambda^{\prime} v_{s}+\mu\right)+v_{u} \lambda^{\prime} A_{\lambda^{\prime}}, \\
m_{\phi_{1}^{0} S}= & -\lambda^{\prime}\left(2 k v_{s}+\mu_{s}\right) v_{d}-4 \lambda^{\prime}\left(\lambda^{\prime} v_{s}+\mu\right) v_{u}+v_{d} \lambda^{\prime} A_{\lambda^{\prime}}, \\
m_{S S}= & m_{S}^{2}+\lambda^{c}\left\{2 k v_{R} \bar{v}_{R}+\lambda^{c}\left(v_{R}^{2}+\bar{v}_{R}^{2}\right)\right\}+54 k_{s}^{2} v_{s}^{2}+\lambda^{\prime}\left\{-2 k v_{d} v_{u}+\lambda^{\prime}\left(v_{d}^{2}+v_{u}^{2}\right)\right\} \\
& +\mu_{s}^{2}+k\left(18 \mu_{s} v_{s}-6 M^{2}\right)+2 \mu_{s} B_{s}+6 v_{s} k A_{k} .
\end{aligned}
$$

This scalar matrix can be diagonalized by the rotation matrix $Z^{H}$ as $Z^{H} \mathbf{m}_{\mathbf{H}}^{2} Z^{H^{\dagger}}=m_{H \text {,diag }}^{2}$. We choose the numerical values of the parameters in such a way that the lightest component becomes the SM-like Higgs boson. We calculate the radiatively corrected Higgs mass up to two loops for the top and stop sector as given in the Ref. [8]. The theoretical error in Higgs boson mass calculation allows for a mass range of 122-128 GeV [32]. In our study, the lightest mass eigenstate for the $C P$-even Higgs boson is mostly composed of the bidoublet scalar fields. This is quite natural as the bidoublet fields are responsible for the EW symmetry breaking once they acquire nonzero VEVs at that scale.

Similarly, the imaginary component of the neutral Higgs fields produces the pseudoscalar $(C P$-odd) states. Their masssquared matrix in the gauge basis $\left(\operatorname{Im}\left[\delta^{c^{0}}\right], \operatorname{Im}\left[\bar{\delta}^{c^{0}}\right], \operatorname{Im}\left[\phi_{2}^{0}\right], \operatorname{Im}\left[\phi_{1}^{0}\right], \operatorname{Im}[S]\right)$ is given as

$$
\mathbf{m}_{\mathbf{A}}^{2}=\left(\begin{array}{ccccc}
M_{\delta^{c}} \delta^{c^{0}} & M_{\bar{\delta}^{c^{0}} \delta^{c^{0}}} & -\lambda^{c} \lambda^{\prime} v_{R} v_{u} & -\lambda^{c} \lambda^{\prime} v_{d} v_{R} & M_{S \delta^{c^{0}}} \\
M_{\delta^{0}} \bar{\delta}^{c^{0}} & M_{\bar{\delta}^{0}} \bar{\delta}^{0} & -\lambda^{c} \lambda^{\prime} \bar{v}_{R} v_{u} & -\lambda^{c} \lambda^{\prime} v_{d} \bar{v}_{R} & M_{S \bar{\delta}^{0}} \\
-\lambda^{c} \lambda^{\prime} v_{R} v_{u} & -\lambda^{c} \lambda^{\prime} \bar{v}_{R} v_{u} & M_{\phi_{2}^{0} \phi_{2}^{0}} & M_{\phi_{1}^{0} \phi_{2}^{0}} & M_{S \phi_{2}^{0}} \\
-\lambda^{c} \lambda^{\prime} v_{d} v_{R} & -\lambda^{c} \lambda^{\prime} v_{d} \bar{v}_{R} & M_{\phi_{2}^{0} \phi_{1}^{0}} & M_{\phi_{1}^{0} \phi_{1}^{0}} & M_{S \phi_{1}^{0}} \\
M_{\delta^{c^{0} S}} & M_{\bar{\delta}^{c^{0} S}} & M_{\phi_{2}^{0} S} & M_{\phi_{1}^{0} S} & M_{S S}
\end{array}\right),
$$

where the elements of the above matrix are

$$
\begin{aligned}
& M_{\delta^{0}{ }^{0} c^{0}}=g_{V}^{2}\left(-v_{R}^{2}+\bar{v}_{R}^{2}\right)+\frac{g_{R}^{2}}{2}\left(2 \bar{v}_{R}^{2}-2 v_{R}^{2}-v_{d}^{2}+v_{u}^{2}\right)+\lambda^{c 2} v_{R}^{2}+m_{1}^{2}+\left(\lambda^{c} v_{s}+\mu_{2}\right)^{2}, \\
& M_{\delta^{0} \bar{c}^{0}}=-\mu_{1}^{*} B_{2}+\lambda^{c}\left(\lambda^{\prime} v_{d} v_{u}-\mu_{s} v_{s}-k v_{s}^{2}+M^{2}\right)-v_{s} \lambda A_{\lambda}, \\
& M_{\bar{\delta}^{c} \bar{\delta}^{c^{0}}}=g_{V}^{2}\left(v_{R}^{2}-\bar{v}_{R}^{2}\right)+\frac{g_{R}^{2}}{2}\left(2 v_{R}^{2}-2 \bar{v}_{R}^{2}-v_{u}^{2}+v_{d}^{2}\right)+\lambda^{c 2} \bar{v}_{R}^{2}+m_{2}^{2}+\left(\lambda^{c} v_{s}+\mu_{2}\right)^{2}, \\
& M_{\phi_{2}^{0} \phi_{2}^{0}}=\lambda^{\prime 2} v_{u}^{2}+\left(\lambda^{\prime} v_{s}+\mu\right)^{2}+\frac{g_{2}^{2}}{4}\left(v_{d}^{2}-v_{u}^{2}\right)+\frac{g_{R}^{2}}{4}\left(2 v_{R}^{2}-2 \bar{v}_{R}^{2}-v_{u}^{2}+v_{d}^{2}\right)+m_{5}^{2}, \\
& M_{\phi_{2}^{0} \phi_{1}^{0}}=\lambda^{\prime}\left(\mu_{s} v_{s}+k v_{s}^{2}+\lambda^{c} v_{R} \bar{v}_{R}-M^{2}\right)+2 v_{s} \lambda^{\prime} A_{\lambda^{\prime}}+2 \mu B, \\
& M_{\phi_{1}^{0} \phi_{1}^{0}}=\lambda^{\prime 2} v_{d}^{2}+\left(\lambda^{\prime} v_{s}+\mu\right)^{2}+\frac{g_{2}^{2}}{4}\left(v_{u}^{2}-v_{d}^{2}\right)+\frac{g_{R}^{2}}{4}\left(2 \bar{v}_{R}^{2}-2 v_{R}^{2}-v_{d}^{2}+v_{u}^{2}\right)+m_{5}^{2}, \\
& M_{\delta^{c^{0} S}}=\lambda^{c} v_{R}\left(2 k v_{s}+\mu_{s}\right)-\lambda A_{\lambda} v_{R}, \\
& M_{\bar{\delta}^{0} S}=\lambda^{c} \bar{v}_{R}\left(2 k v_{s}+\mu_{s}\right)-\lambda A_{\lambda} \bar{v}_{R} \text {, } \\
& M_{\phi_{2}^{0} S}=-\lambda^{\prime} v_{u}\left(2 k v_{s}+\mu_{s}\right)+2 v_{u} \lambda^{\prime} A_{\lambda^{\prime}}, \\
& M_{\phi_{1}^{0} S}=-\lambda^{\prime} v_{d}\left(2 k v_{s}+\mu_{s}\right)+2 v_{d} \lambda^{\prime} A_{\lambda^{\prime}} \\
& M_{S S}=m_{S}^{2}-2 \lambda^{c} k v_{R} \bar{v}_{R}+\lambda^{c 2}\left(v_{R}^{2}+\bar{v}_{R}^{2}\right)+18 k_{s}^{2} v_{s}^{2}+2 \lambda^{\prime} k v_{d} v_{u}+\lambda^{\prime 2}\left(v_{d}^{2}+v_{u}^{2}\right)+\mu_{s}^{2} \\
& +2 k\left(\mu_{s} v_{s}+M^{2}\right)-2 \mu_{s} B_{s}-6 v_{s} k A_{k} .
\end{aligned}
$$

This pseudoscalar mass-squared matrix can be diagonalized by the rotation matrix $Z^{A}$ as $Z^{A} \mathbf{m}_{\mathbf{A}}^{2} Z^{A^{\dagger}}=m_{A \text {,diag }}^{2}$. After rotating the gauge fields into mass basis, we get three physical mass eigenstates $A_{1}, A_{2}$, and $A_{3}$. The remaining two neutral states $\left(G_{1}^{0}, G_{2}^{0}\right)$ become the massless Goldstone bosons that are absorbed as the longitudinal components of the corresponding gauge bosons $Z$ and $Z_{R}$, respectively. 


\section{Sfermion sectors}

The sfermions in our model consist of the scalar superpartners of the up- and down-type quarks and charged and neutral leptons. The existence of a right-handed neutrino and hence its superpartner is guaranteed by the extended gauge symmetry in this model, which leads to all the sfermion mass-squared matrices (including sneutrinos) being $6 \times 6$ matrices in general. We calculate the masssquared matrices for the scalar down-type squarks, up-type squark, charged slepton, and sneutrino in the $\left(\tilde{d}_{L}, \tilde{d}_{R}\right)$,
$\left(\tilde{u}_{L}, \tilde{u}_{R}\right),\left(\tilde{e}_{L}, \tilde{e}_{R}\right)$, and $\left(\tilde{\nu}_{L}, \tilde{\nu}_{R}\right)$ gauge basis, respectively. Thus, one can write the mass-squared matrices for the squarks as

$$
\begin{aligned}
& \mathbf{m}_{\tilde{\mathbf{d}}}^{2}=\left(\begin{array}{ll}
m_{\tilde{d}_{L} \tilde{d}_{L}^{*}} & m_{\tilde{d}_{L} \tilde{d}_{R}^{*}} \\
m_{\tilde{d}_{L} \tilde{d}_{R}^{*}}^{\dagger} & m_{\tilde{d}_{R} \tilde{d}_{R}^{*}}
\end{array}\right) \\
& \mathbf{m}_{\tilde{\mathbf{u}}}^{2}=\left(\begin{array}{ll}
m_{\tilde{u}_{L} \tilde{u}_{L}^{*}} & m_{\tilde{u}_{L} \tilde{u}_{R}^{*}} \\
m_{\tilde{u}_{L} \tilde{u}_{R}^{*}}^{\dagger} & m_{\tilde{u}_{R} \tilde{u}_{R}^{*}}^{*}
\end{array}\right),
\end{aligned}
$$

where each matrix element is itself a $3 \times 3$ matrix given as

$$
\begin{aligned}
& m_{\tilde{d}_{L} \tilde{d}_{L}^{*}}=\delta_{\alpha_{1} \beta_{1}}\left[v_{d}^{2} y_{q} y_{q}^{T}+M_{Q L}^{2}+\frac{g_{V}^{2}}{6}\left(v_{R}^{2}-\bar{v}_{R}^{2}\right)+\frac{g_{2}^{2}}{4}\left(v_{u}^{2}-v_{d}^{2}\right)\right], \\
& m_{\tilde{d}_{R} \tilde{d}_{R}^{*}}=\delta_{\alpha_{2} \beta_{2}}\left[v_{d}^{2} y_{q}^{T} y_{q}+M_{Q R}^{2}+\frac{g_{V}^{2}}{6}\left(\bar{v}_{R}^{2}-v_{R}^{2}\right)+\frac{g_{R}^{2}}{4}\left(2 \bar{v}_{R}^{2}-2 v_{R}^{2}-v_{d}^{2}+v_{u}^{2}\right)\right], \\
& m_{\tilde{d}_{L} \tilde{d}_{R}^{*}}=\delta_{\alpha_{1} \beta_{2}}\left[\left(\lambda^{\prime} v_{s}+\mu\right) v_{u} y_{q}-v_{d} \lambda T^{y q}\right], \\
& m_{\tilde{u}_{L} \tilde{u}_{L}^{*}}=\delta_{\alpha_{1} \beta_{1}}\left[v_{u}^{2} y_{q} y_{q}^{T}+M_{Q L}^{2}+\frac{g_{V}^{2}}{6}\left(v_{R}^{2}-\bar{v}_{R}^{2}\right)+\frac{g_{2}^{2}}{4}\left(v_{d}^{2}-v_{u}^{2}\right)\right], \\
& m_{\tilde{u}_{R} \tilde{u}_{R}^{*}}=\delta_{\alpha_{2} \beta_{2}}\left[v_{u}^{2} y_{q}^{T} y_{q}+M_{Q R}^{2}+\frac{g_{V}^{2}}{6}\left(\bar{v}_{R}^{2}-v_{R}^{2}\right)+3 \frac{g_{R}^{2}}{4}\left(2 v_{R}^{2}-2 \bar{v}_{R}^{2}-v_{u}^{2}+v_{d}^{2}\right)\right], \\
& m_{\tilde{u}_{L} \tilde{u}_{R}^{*}}=\delta_{\alpha_{1} \beta_{2}}\left[-v_{d}\left(\lambda^{\prime} v_{s}+\mu\right) y_{q}+v_{u} T^{y q}\right] .
\end{aligned}
$$

Here $\alpha_{1}, \alpha_{2}, \beta_{1}$, and $\beta_{2}$ represent the color indices. These $3 \times 3$ matrices in general can be nondiagonal with the off-diagonal elements allowing for mixing between the various flavors. We do not consider a flavor violating process in our study and hence, for simplicity, we just consider the case where the matrices are diagonal and their elements are real.

Similarly, the slepton mass-squared matrices are given as

$$
\begin{aligned}
& \mathbf{m}_{\tilde{\mathbf{e}}}^{2}=\left(\begin{array}{ll}
m_{\tilde{e}_{L}} \tilde{e}_{L}^{*} & m_{\tilde{e}_{L} \tilde{e}_{R}} \\
m_{\tilde{e}_{L}}^{\dagger} \tilde{e}_{R}^{*} & m_{\tilde{e}_{R} \tilde{e}_{R}^{*}}
\end{array}\right) \\
& \mathbf{m}_{\tilde{\nu}}^{2}=\left(\begin{array}{ll}
m_{\tilde{\nu}_{L} \tilde{L}_{L}^{*}} & m_{\tilde{\nu}_{L} \tilde{\nu}_{R}} \\
m_{\tilde{\nu}_{L} \tilde{L}_{R}^{*}}^{\dagger} & m_{\tilde{\nu}_{R} \tilde{\nu}_{R}^{*}}
\end{array}\right),
\end{aligned}
$$

with the matrix elements in the slepton sector being

$$
\begin{aligned}
& m_{\tilde{e}_{L} \tilde{e}_{L}^{*}}=\frac{g_{V}^{2}}{2}\left(\bar{v}_{R}^{2}-v_{R}^{2}\right)+\frac{g_{2}^{2}}{4}\left(v_{u}^{2}-v_{d}^{2}\right)+v_{d}^{2} y_{l} y_{l}^{T}+M_{L L}^{2}, \\
& m_{\tilde{e}_{R} \tilde{e}_{R}^{*}}=\frac{g_{V}^{2}}{2}\left(v_{R}^{2}-\bar{v}_{R}^{2}\right)+\frac{g_{R}^{2}}{4}\left(2 \bar{v}_{R}^{2}-2 v_{R}^{2}-v_{d}^{2}+v_{u}^{2}\right)+v_{d}^{2} y_{l}^{T} y_{l}+M_{L R}^{2}, \\
& m_{\tilde{e}_{L} \tilde{e}_{R}}=y_{l}\left(\lambda^{\prime} v_{s}+\mu\right) v_{u}-v_{d} T^{y l}, \\
& m_{\tilde{\nu}_{L} \tilde{\nu}_{L}^{*}}=\frac{g_{V}^{2}}{2}\left(\bar{v}_{R}^{2}-v_{R}^{2}\right)+\frac{g_{2}^{2}}{4}\left(v_{d}^{2}-v_{u}^{2}\right)+v_{u}^{2} y_{l} y_{l}^{T}+M_{L L}^{2}, \\
& m_{\tilde{\nu}_{R} \tilde{\nu}_{R}^{*}}=\bar{v}_{R}^{2} f^{c T} f^{c}+\frac{g_{V}^{2}}{2}\left(v_{R}^{2}-\bar{v}_{R}^{2}\right)+\frac{g_{R}^{2}}{4}\left(2 v_{R}^{2}-2 \bar{v}_{R}^{2}-v_{u}^{2}+v_{d}^{2}\right)+v_{u}^{2} y_{l}^{T} y_{l}+M_{L R}^{2}, \\
& m_{\tilde{\nu}_{L} \tilde{\nu}_{R}}=-y_{l} v_{d}\left(\lambda^{\prime} v_{s}+\mu\right)+v_{u} T^{y l} .
\end{aligned}
$$

The sfermion mass matrices, namely, the down squarks, up squarks, charged sleptons, and sneutrinos given in Eqs. (24)-(28), can be diagonalized by the rotation matrices $U^{D L}, U^{U L}, U^{E L}$, and $U^{V L}$, respectively. 


\section{Electroweakino sectors}

The particle spectrum of our model allows for a large number of physical chargino and neutralino states (together referred to as electroweakinos from here on), which arise from the mixing of the charged and neutral gauginos and Higgsinos, respectively. Since R parity is naturally conserved in this model, the lightest neutralino is stable and can be a good dark matter candidate. The electroweakinos are also very important for our study as the primary SUSY decay channels for the heavy gauge bosons will be into these particles, as is seen in the next section.

The chargino mass matrix in the basis $\left(\tilde{W}_{R}^{-}, \tilde{W}_{L}^{-}, \tilde{\delta}^{c^{-}}, \tilde{\phi}_{2}^{-}\right)$ and $\left(\tilde{W}_{R}^{+}, \tilde{W}_{L}^{+}, \tilde{\bar{\delta}}^{c^{+}}, \tilde{\phi}_{2}^{+}\right)$can be written as

$$
\mathbf{m}_{\chi_{\mathrm{c}}}=\left(\begin{array}{llll}
\tilde{W}_{R}^{-} & \tilde{W}_{L}^{-} & \tilde{\delta}^{c^{-}} & \tilde{\phi}_{2}^{-}
\end{array}\right)\left(\begin{array}{cccc}
\frac{M_{11}^{W_{R}}+M_{22}^{W_{R}}}{2} & 0 & \sqrt{2} g_{R} v_{R} & g_{R} v_{d} \\
0 & \frac{1 M_{11}^{W_{L}}+M_{22}^{W_{L}}}{2} & 0 & g_{2} v_{u} \\
-\sqrt{2} g_{R} \bar{v}_{R} & 0 & \lambda^{c} v_{s}+\mu_{2} & 0 \\
g_{R} v_{u} & g_{2} v_{d} & 0 & \left(\lambda^{\prime} v_{s}+\mu\right)
\end{array}\right)\left(\begin{array}{c}
\tilde{W}_{R}^{+} \\
\tilde{W}_{L}^{+} \\
\tilde{\tilde{\delta}}^{c^{+}} \\
\tilde{\phi}_{2}^{+}
\end{array}\right) .
$$

It is easy to see that this chargino mass matrix is asymmetric and can only be diagonalized by a biunitary transformation with $m_{\tilde{\chi}_{c} \text {,iag }}=U^{L m} \mathbf{m}_{\tilde{\chi}_{\mathrm{c}}} U^{R p^{\dagger}}$. Please note that the left-handed triplet Higgsinos remain decoupled from these charginos and neutralinos due to the left-handed triplet Higgs boson not acquiring any VEV. Hence we have a total of eight neutral electroweakinos that mix among each other in the gauge basis

$$
\left(\tilde{B}, \widetilde{W_{R}^{0}}, \widetilde{W_{L}^{0}}, \tilde{\delta}^{c^{0}}, \tilde{\bar{\delta}}^{c^{0}}, \tilde{\phi}_{2}^{0}, \tilde{\phi}_{1}^{0}, \tilde{S}\right)
$$

with their corresponding mass matrix as

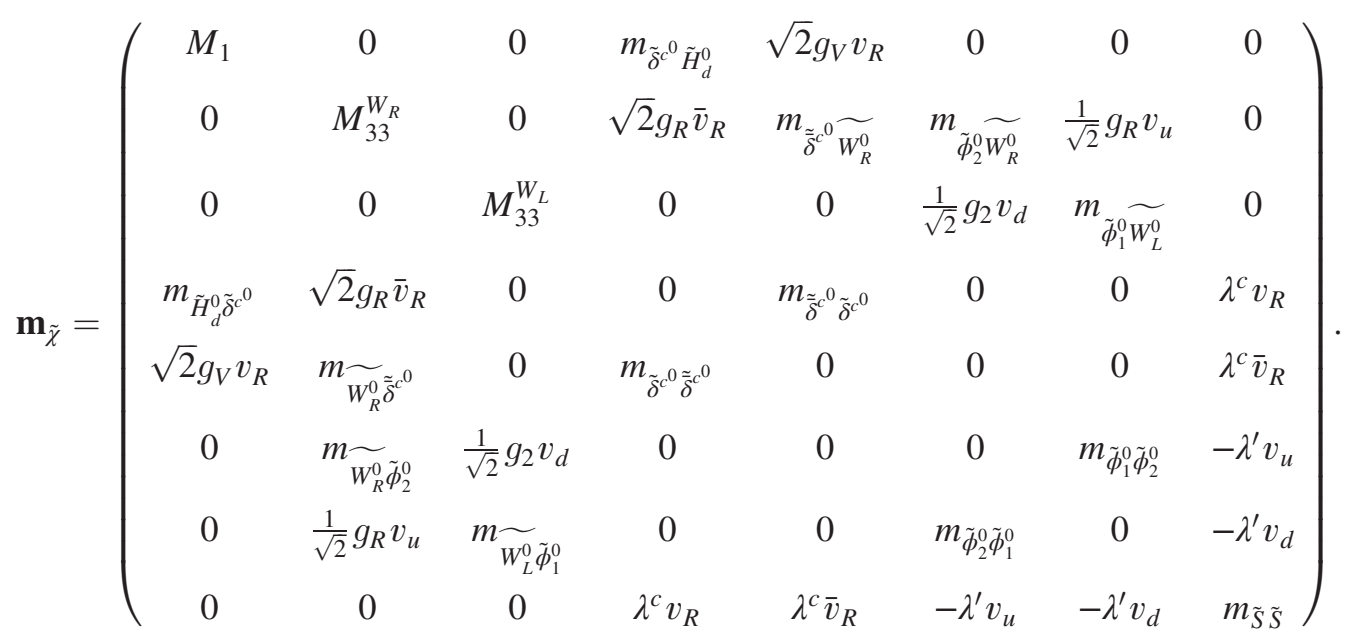

Here

$$
\begin{array}{ll}
m_{\tilde{H}_{d}^{0} \tilde{c}^{0}}=-\sqrt{2} g_{V} \bar{v}_{R}, \quad m_{\widetilde{W_{R}^{0}} \tilde{\delta} c^{0}}=-\sqrt{2} g_{R} v_{R}, \quad m_{\tilde{\delta}^{c} \tilde{\delta}^{0}}=\lambda^{c} v_{s}+\mu_{2}, \quad m_{W_{R}^{0} \tilde{\phi}_{2}^{0}}=-\frac{1}{\sqrt{2}} g_{R} v_{d}, \\
m \widetilde{W_{L}^{0} \tilde{\phi}_{1}^{0}}=-\frac{1}{\sqrt{2}} g_{2} v_{u}, \quad m_{\tilde{\phi}_{2}^{0} \tilde{\phi}_{1}^{0}}=-\left(\lambda^{\prime} v_{s}+\mu\right), \quad m_{\tilde{S} \tilde{S}}=\left(2 k v_{s}+\mu_{s}\right) .
\end{array}
$$

This matrix is diagonalized by the $Z^{f N}$ rotation matrix as $m_{\tilde{\chi} \text {,diag }}=Z^{f N} \mathbf{m}_{\tilde{\chi}} Z^{f N^{\dagger}}$. Also in this model, it is possible to get two types of doubly charged chargino particles, one from the $S U(2)_{L}$ triplet and another from $S U(2)_{R}$ triplet sectors. These do not mix among each other, resulting in the left-handed triplets being quite massive while the right-handed doubly charged Higgsinos can remain light [33] with a mass of

$$
M_{\tilde{\chi}^{ \pm \pm}}=\lambda^{c} v_{s}+\mu_{2}
$$




\section{HEAVY GAUGE BOSON DECAYS FOR DIFFERENT LSP COMPOSITIONS}

In this study, we mainly concentrate on the heavy $W_{R}$ and $Z_{R}$ gauge bosons. Depending on the numerical values of the additional gauge couplings $g_{R}, g_{V}$ and the vacuum expectation values, i.e., the minimum of the potential in the particular scalar field directions (except the singlet scalar field), the masses of these gauge bosons could change. In the presence of light LRSUSY particles, and if kinematically allowed, these heavy gauge bosons can decay to these particles with a significantly high branching fraction. In order to estimate these BSM decays, we choose the parameters in such a way that all the sparticle sectors are sufficiently heavy except the electroweakinos [see Eq. (30)] so that the heavy gauge bosons can only decay into these light electroweakinos. We specifically focus on this sector as the SUSY decays of the heavy gauge bosons is maximum here. First, we consider different benchmark points where the light electroweakinos are primarily composed of only one type of fermionic fields (a particular type of gaugino or Higgsino). Then we allow for mixing between the various fermionic states such that the decay branching fractions of the gauge bosons can change significantly. We also vary the masses of the squarks and sleptons so as to open up the gauge bosons decay channels into sfermionic final states. Thus in this section we study a host of scenarios with various possible final states for the heavy gauge boson decays and study the corresponding decay BRs.

A systematic study of the heavy gauge bosons decay channels requires one to deal with a large number of free parameters in the minimal LRSUSY model that has been considered in the paper. The experimentally measured particle masses and other low energy observables can be used to constrain the Yukawa sector of the model to a certain extent. The scalar couplings, on the other hand, have some bounds coming from the measured Higgs boson properties. We further require the lightest neutralino to be the LSP as it can then be a good dark matter candidate. Yet there are a large number of free parameters in the model, most of which do not have any significant effect on our results. We thus keep the numerical values of these parameters constant for the rest of our analysis as can be seen in Table I. Varying the rest of the parameters, we consider different field combinations for the electroweakino sector while also varying the sfermion masses to study the corresponding gauge boson decays.

\section{A. Case-1: Single component LSP}

We first identify the parameter spaces where the LSP is mostly composed of only one type of component among the neutral fermion fields in the basis given in Eq. (30). We make sure all other SUSY particles are much heavier so that the heavy gauge bosons do not decay into these states. It can be seen from Eq. (7) that the mass of the neutral $Z_{R}$ boson always remains $\sqrt{2\left(1+g_{R}^{2} g_{V}^{-2}\right)}$ times heavier than the mass of the $W_{R}$ boson. We keep the numerical values of the VEVs fixed at $v_{u}=173.457 \mathrm{GeV}, v_{d}=15 \mathrm{GeV}$, $v_{R}=7300 \mathrm{GeV}, \bar{v}_{R}=3730 \mathrm{GeV}$, and $v_{s}=1000 \mathrm{GeV}$ and the gauge couplings $g_{V}=0.36, g_{2}=g_{R}=0.64$. Thus the masses of the heavy gauge bosons remain unchanged at $M_{W_{R}}=5.25 \mathrm{TeV}$ and $M_{Z_{R}}=8.5 \mathrm{TeV}$ throughout this section. One could also choose different values of the VEVs and the gauge couplings to get different gauge boson masses. We however choose relatively heavy masses for the gauge bosons to evade the experimental bounds $[12,16,18]$. As discussed earlier, we have fixed the numerical values of a number of parameters in the model, which are shown in Table I. Note that the large values of the right-handed Yukawa couplings $f_{i i}^{c}$ in the table result in the right-handed neutrinos being heavier than the $W_{R}$ boson mass. Thus it is impossible for the heavy gauge bosons to decay into right-handed neutrino final states. The dominant

TABLE I. These parameters remain fixed throughout this section. The unit of the mass parameter is in GeV and mass squared is in $\mathrm{GeV}^{2}$.

\begin{tabular}{l}
\hline $\bar{c}$ Parameters \\
\hline$M_{q L, 11}^{2}=M_{q L, 22}^{2}=10^{8}, M_{q L, 33}^{2}=2.50 \times 10^{7}, M_{q R, 11}^{2}=M_{q R, 22}^{2}=10^{8}, M_{q R, 33}^{2}=2.90 \times 10^{7}$, \\
$M_{l L, 11}^{2}=9.60 \times 10^{7}, M_{l L, 22}^{2}=9.20 \times 10^{7}, M_{l L, 33}^{2}=9.0 \times 10^{7}, M_{L R, 11}^{2}=9.20 \times 10^{7}, M_{L R, 22}^{2}=9.10 \times 10^{7}, M_{L R, 33}^{2}=9.0 \times 10^{7}$, \\
$y_{l, 11}=3.34 \times 10^{-5}, y_{l, 22}=0.007, y_{l, 33}=0.118, T_{11}^{y l}=T_{22}^{y l}=T_{33}^{y l}=-100$, \\
$y_{l, i j}=0=T_{i j}^{y l}$, for $i \neq j$, \\
$y_{q, 11}=1.45 \times 10^{-5}, y_{q, 22}=0.0073, y_{q, 33}=1.0006, T_{11}^{y q}=T_{22}^{y q}=T_{33}^{y q}=-100$, \\
$y_{q, i j}=0=T_{i j}^{y q}$, for $i \neq j$, \\
$f_{11}^{c}=1.4, f_{22}^{c}=1.6, f_{33}^{c}=1.8, T_{11}^{f^{c}}=T_{22}^{f^{c}}=T_{33}^{f^{c}}=-100$, \\
$f_{i j}^{c}=0=T_{i j}^{f^{c}}$, for $i \neq j$. \\
$\lambda^{\prime}=0.10, k=1.8$, \\
$\mu_{1}^{*} B_{2}=-2000, \mu_{s} B_{s}=10^{3}, M^{2}=-10^{8}, \lambda C_{\lambda}=10^{3}, \lambda A_{\lambda}=-100, k A_{k}=10^{4}, \lambda^{\prime} A_{\lambda^{\prime}}=-350, M_{G}=6000$, \\
$v_{u}=173.457, v_{d}=15, v_{R}=7300, \bar{v}_{R}=3730, v_{s}=1000, g_{V}=0.36, g_{2}=g_{R}=0.64$ \\
\hline \hline
\end{tabular}


TABLE II. The LSP is mostly composed of only one type of component among the neutral fermion fields in the basis given in Eq. (30). $M_{\tilde{\chi}_{i}}\left(\tilde{\chi}_{i}=\tilde{\chi}_{1,2}^{0}, \tilde{\chi}_{1}^{ \pm}\right)$stands for the masses of the electroweakinos for these benchmark points. It is to be noted that the other parameters are fixed as in Table I. The decay into BSM particles having branching ratios less than $0.0001 \%$ is not included here.

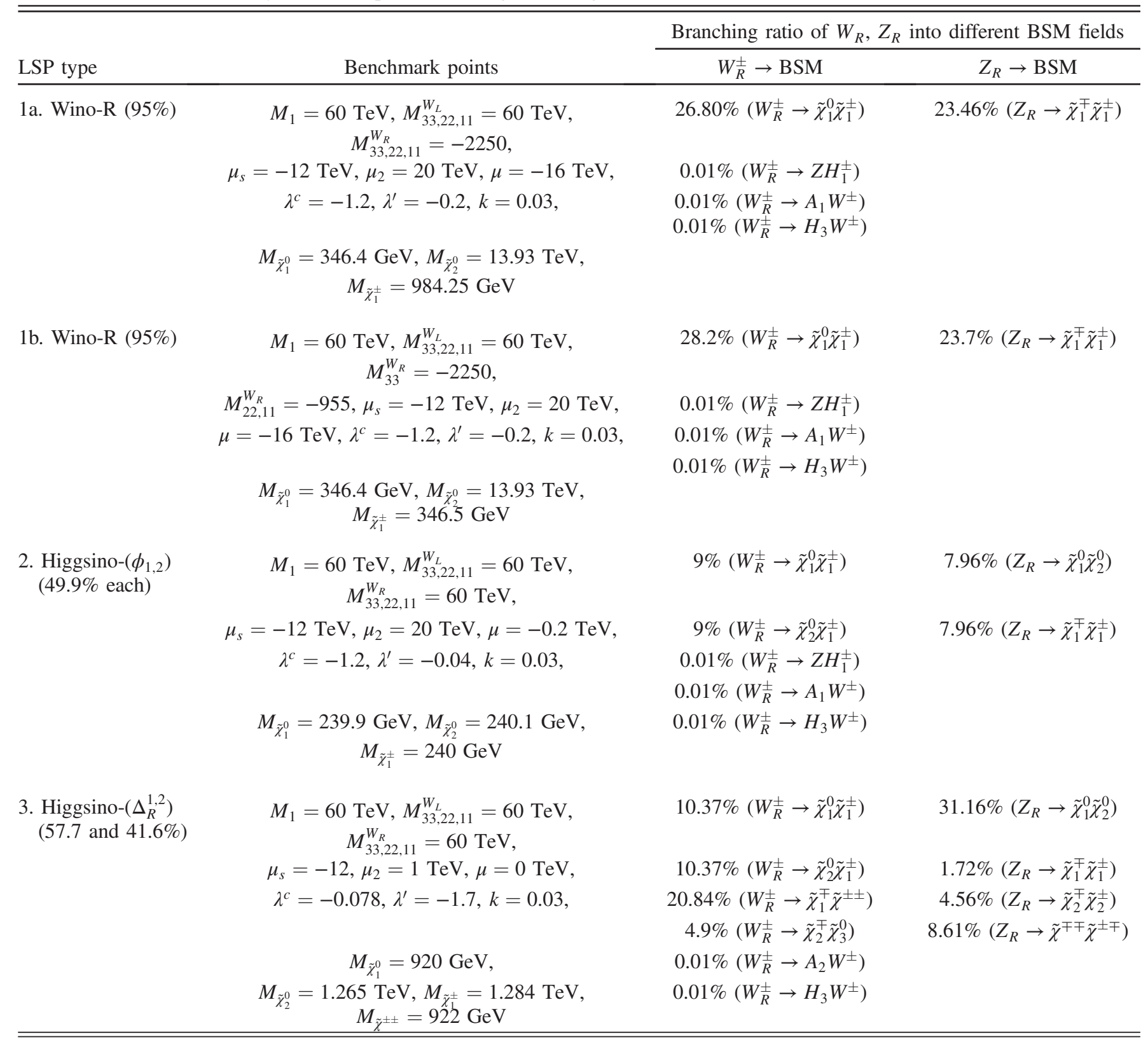

contribution to the LSP from each fermion field is shown in Table II and is discussed in detail below.

\section{Binolike, $S U(2)_{L}$, winolike, or singlinolike LSP}

One can consider the case where the neutralino LSP is mostly composed of $\tilde{B}, \tilde{W}_{L}^{0}$, or $\tilde{S}$. As is quite evident from the neutralino mass matrix given in Eq. (31), a bino(winoL)-type LSP would require one to choose a small value of the parameter $M_{1}\left(M_{33}^{W_{L}}\right)$. Similarly one can adjust $\lambda$ 's and $\mu$ 's to get a singlinolike LSP. These scenarios result in the $\mathrm{BR}$ of heavy gauge bosons decaying into BSM particles being almost negligible and do not give rise to any new decay channels. The heavy gauge bosons here almost entirely decay into SM particles resulting in no new signals, and hence are not discussed in detail.

\section{2. $S U(2)_{R}$ winolike LSP}

As a consequence of the right-handed symmetry being broken above the SUSY breaking scale, one can choose different values of the soft SUSY breaking terms for the charged and the neutral components of the $S U(2)_{R}$ wino fields. The parameter $M_{33}^{W_{R}}$ can thus be adjusted to get a 
neutralino LSP primarily consisting of a neutral $S U(2)_{R}$ wino (or wino-R) field. Again, in this case, due to the offdiagonal $(2,4)$ and $(2,5)$ entries in the neutralino mass matrix $\left[\sqrt{2} g_{R} \bar{v}_{R}\right.$ and $\sqrt{2} g_{R} v_{R}$ respectively as can be seen from Eq. (31)], the lightest neutralino mass $M_{\tilde{\chi}_{1}^{0}} \geq$ $2.29 \mathrm{TeV}$ for any positive value of $M_{33}^{W_{R}}$ for our chosen parameters. Similarly for positive values of the parameters $M_{i i}^{W_{R}}(i=1,2)$, the wino-Rlike chargino mass remains $M_{\tilde{\chi}_{1}^{ \pm}} \geq 1.11 \mathrm{TeV}$ due to the off-diagonal $(1,3)$ and $(3,1)$ terms in the chargino mass matrix given in Eq. (29). In fact if all the three soft terms are equal and positive, the chargino mass always remains lighter than the neutralino mass for an $S U(2)_{R}$ winolike LSP. Thus we are compelled to choose either negative or unequal numerical values for $M_{i i}^{W_{R}}(i=1-3)$. To analyze the distinct regions in the parameter space, we choose two corresponding BPs 1(a) and 1(b) as shown in Table II. The right-handed neutrinos, squarks, and sleptons masses are all heavier than the $W_{R}$ and $Z_{R}$ boson masses so these final states are absent here.

For BP 1(a) and 1(b) we keep the numerical value of $M_{33}^{W_{R}}=-2250 \mathrm{GeV}$ the same while the charged wino-R soft masses $M_{11}^{W_{R}}=M_{22}^{W_{R}}$ are chosen to be $-2250 \mathrm{GeV}$ for BP 1(a) and $-955 \mathrm{GeV}$ for BP 1(b). This gives us a lightest chargino mass of 984.25 and $346.5 \mathrm{GeV}$ for the two cases, respectively. The lightest neutralino mass remains at 346.4 GeV for all the three benchmarks we have considered here. The $W_{R}^{ \pm} \rightarrow \tilde{\chi}_{1}^{0} \tilde{\chi}_{1}^{ \pm}$and $Z_{R} \rightarrow \tilde{\chi}_{1}^{ \pm} \tilde{\chi}_{1}^{\mp}$ channels open up for these two BPs, resulting in significant branching ratios into these decay channels. Both these cases have similar couplings for $W_{R}^{ \pm} \tilde{\chi}_{1}^{0} \tilde{\chi}_{1}^{\mp}$, which come out $\sim\left(0.56 P_{L}+0.58 P_{R}\right)$ while the $Z_{R} \tilde{\chi}_{1}^{ \pm} \tilde{\chi}_{1}^{\mp}$ coupling is $\sim\left(0.50 P_{L}+0.53 P_{R}\right)$, where $P_{L}$ and $P_{R}$ are the left and right chiral projection operators of the fermions. As a result the branching ratio of $W_{R}^{ \pm} \rightarrow \tilde{\chi}_{1}^{0} \tilde{\chi}_{1}^{ \pm}$is around $26.8 \%$ while $Z_{R} \rightarrow \tilde{\chi}_{1}^{ \pm} \tilde{\chi}_{1}^{\mp}$ remains around $23.46 \%$ for both BP 1 (a) and 1 (b) as can be seen from Table II. The branching ratios to the other Higgs boson and/or vector boson final state decay channels remain negligibly small.

\section{Bidoublet Higgsino-like LSP}

We now consider the case where the LSP is primarily composed of the bidoublet Higgsino fields. This can be achieved by choosing a relatively small value for the offdiagonal bidoublet Higgsino cross term $\left|\frac{\lambda^{\prime}}{2} v_{s}+\frac{\mu}{2}\right|$ while keeping the other mass terms in the neutralino mass matrix quite large. As the diagonal mass terms for $\tilde{\phi}_{1}^{0}$ and $\tilde{\phi}_{2}^{0}$ in the $\mathbf{m}_{\tilde{\chi}}$ matrix are 0 , their entire mass comes from the offdiagonal term mentioned above. This results in two light neutralino mass eigenstates that are almost degenerate in mass and composed of maximally mixed states of $\tilde{\phi}_{1}^{0}$ and $\tilde{\phi}_{2}^{0}$ while their mixing with the other states is negligibly small. Hence, both the LSP and the next-to-lightest SUSY particle (NLSP) arise from an equal mixing $(\sim 49.9 \%)$ of $\tilde{\phi}_{1}^{0}$ and $\tilde{\phi}_{2}^{0}$, with their masses being 239.9 and $240.1 \mathrm{GeV}$, respectively. The lightest chargino also primarily consists of bidoublet charged fields and has a mass of $240 \mathrm{GeV}$. Our choice of parameters (the BP 2 in Table II) directly affects the tree-level and loop-level Higgs mass; we thus slightly modify the numerical value of $\lambda^{\prime} A_{\lambda^{\prime}}$ to get the lightest Higgs mass of $M_{h_{1}}=125 \mathrm{GeV}$.

The branching ratio of $W_{R}^{ \pm} \rightarrow \tilde{\chi}_{1}^{0} \tilde{\chi}_{1}^{ \pm}$and $W_{R}^{ \pm} \rightarrow \tilde{\chi}_{2}^{0} \tilde{\chi}_{1}^{ \pm}$ becomes identical (9\% in each channel) since their coupling strengths $\left(\sim 0.31 P_{L}+0.31 P_{R}\right)$ are also equal here. This is because the composition of $\tilde{\chi}_{1}^{0}$ and $\tilde{\chi}_{2}^{0}$ is almost the same. The $\tilde{\phi}_{1}^{0} \tilde{\phi}_{1}^{0} Z_{R}$ and $\tilde{\phi}_{2}^{0} \tilde{\phi}_{2}^{0} Z_{R}$ terms in the gauge basis appear with equal and opposite sign couplings and as a result, in the mass basis, the $Z_{R} \tilde{\chi}_{1}^{0} \tilde{\chi}_{1}^{0}$ and $Z_{R} \tilde{\chi}_{2}^{0} \tilde{\chi}_{2}^{0}$ couplings vanish in this case. This fact can also be seen from Eq. (B16). Hence, these are suppressed (as $\tilde{\chi}_{1,2}$ has tiny contributions from the other neutral Higgsino and gaugino fields) and the corresponding decay BRs remain negligibly small. However, the vertices $Z_{R} \tilde{\chi}_{1}^{0} \tilde{\chi}_{2}^{0}$ and $Z_{R} \tilde{\chi}_{1}^{ \pm} \tilde{\chi}_{1}^{ \pm}$have quite large couplings resulting in a sizable branching ratio of around $8 \%$ in each of these channels.

\section{Right-handed triplet Higgsino-like LSP}

The $S U(2)_{R}$ triplet Higgsino-like neutralino LSP can be obtained by adjusting the value of the parameter $\mu_{2}, \lambda^{c}$, and $v_{s}$ appearing in the $(4,5)$ and the $(5,4)$ matrix elements of the neutralino mass matrix given in Eq. (31). We change $\mu_{2}$ and $\lambda^{c}$ while keeping $v_{s}$ constant. As these parameters directly affect the tree-level Higgs mass, we adjust the loopcorrected and tree-level Higgs mass by appropriately choosing the value of the $M_{q L / R}, \lambda^{\prime} A_{\lambda^{\prime}}, \mu$, and $\lambda^{\prime}$ parameters to achieve a lightest Higgs boson mass of $125 \mathrm{GeV}$. In this case, we can get large splitting between the neutralinos $\tilde{\chi}_{1}^{0}$ and $\tilde{\chi}_{2}^{0}$ due to the large off-diagonal $(2,4),(2,5),(4,2)$, and $(5,2)$ elements of the neutralino mass matrix [see Eq. (31)]. This also allows us to have quite different contributions from $\tilde{\delta}^{c^{0}}$ and $\tilde{\bar{\delta}}^{c^{0}}$ in the $\tilde{\chi}_{1}^{0}$ eigenstate unlike the previous case of bidoublet Higgsinos. We present this case as BP 3 in Table II. The light neutralino masses are $M_{\tilde{\chi}_{1}^{0}}=920 \mathrm{GeV}$ and $M_{\tilde{\chi}_{2}^{0}}=1265 \mathrm{GeV}$ while the lightest chargino mass is $M_{\tilde{\chi}_{1}^{ \pm}}=1284 \mathrm{GeV}$ for this benchmark point. An important consequence of our chosen benchmark point is that the doubly charged Higgsino also remains light here as it is a part of the same fermion multiplet that gives the LSP in this scenario. We get the mass of the doubly charged Higgsino $M_{\tilde{\chi}^{ \pm \pm}}=922 \mathrm{GeV}$.

As $\tilde{\chi}_{1}^{0}, \tilde{\chi}_{2}^{0}$, and $\tilde{\chi}_{1}^{ \pm}$are all composed of right-handed triplet states, their couplings with $W_{R}$ are relatively large compared to other cases. The coupling strengths of $W_{R}^{ \pm} \tilde{\chi}_{1}^{0} \tilde{\chi}_{1}^{ \pm}$ vertex are $\sim 0.45 P_{L}+0.39 P_{R}$ while that of $W_{R}^{ \pm} \tilde{\chi}_{2}^{0} \tilde{\chi}_{1}^{ \pm}$vertex is $\sim 0.39 P_{L}+0.45 P_{R}$ [see the second term of both the lines of Eq. (A12)] for the BP 3. Thus the branching ratios of 
$W_{R}^{ \pm} \rightarrow \tilde{\chi}_{1}^{0} \tilde{\chi}_{1}^{ \pm}$and $W_{R}^{ \pm} \rightarrow \tilde{\chi}_{2}^{0} \tilde{\chi}_{1}^{ \pm}$are almost the same here and equal to around $10.37 \%$ each. The doubly charged fermion being light allows an additional decay channel for $W_{R}^{ \pm} \rightarrow$ $\tilde{\chi}_{1}^{\mp} \tilde{\chi}^{ \pm \pm}$and gives an even larger branching ratio of $20.84 \%$ for this channel owing to the larger coupling of the $W_{R}^{ \pm} \tilde{\chi}_{1}^{\mp} \tilde{\chi}^{ \pm \pm}$vertex as given in Eq. (A13).

The neutral $Z_{R}$ boson has several possible decay channels with electroweakino final states in this case. The $\tilde{\delta}^{c^{0}} \tilde{\delta}^{c^{0}} Z_{R}$ and $\tilde{\bar{\delta}}^{c^{0}} \tilde{\bar{\delta}}^{c^{0}} Z_{R}$ terms in the gauge basis appear with equal and opposite sign as in the previous case. Hence the $Z_{R} \tilde{\chi}_{1}^{0} \tilde{\chi}_{1}^{0}$ and $Z_{R} \tilde{\chi}_{2}^{0} \tilde{\chi}_{2}^{0}$ couplings are again small due to cancellations in the mass basis. On the other hand, the $Z_{R} \rightarrow \tilde{\chi}_{1}^{0} \tilde{\chi}_{2}^{0}$ channel has a large branching ratio of $31.16 \%$ due to a large coupling of $\sim 0.61 P_{L}+0.61 P_{R}$ [see Eq. (B16)] in this vertex. The $Z_{R} \rightarrow \tilde{\chi}_{1}^{ \pm} \tilde{\chi}_{1}^{\mp}$ remains quite small at around $2 \%$. A sizable $8.61 \%$ branching in the channel $Z_{R} \rightarrow \tilde{\chi}^{\mp \mp} \tilde{\chi}^{ \pm \pm}$is also obtained in this scenario.

\section{B. Case II: Mixed LSP}

In the previous section, we analyzed the regions of the parameter space where the major contribution to the LSP was primarily coming from only one type of component of the neutral gaugino or Higgsino fields. In this section, we discuss the parameter space where the LSP consists of two type of components [see Eq. (30)] and study its effect on the heavy gauge boson decay channels. As before, the rest of the BSM particle spectrum (squarks, sleptons, right-handed neutrinos, etc.,) have been kept much heavier to make sure that the heavy gauge bosons do not decay into them. Cases with dominant contribution to the LSP from two gaugino fields are shown in Table III while the cases with mixed gaugino and Higgsino fields relevant for our study are presented in Table IV. The Higgsinos, as well, can mix among each other, resulting in the LSP being composed of equal parts coming from the bidoublet fields and the righthanded triplet fields. The choice of parameters leading to this mixed Higgsino-like LSP is given in Table V. We now present a detailed discussion of each of these cases.

\section{Combination of bino, wino $L$, and/or singlino}

The LSP comprising a mixture of the bino, wino-L field, and/or singlino can be found by adjusting the parameters $M_{1}, M_{33}^{W_{L}}, \lambda^{c}, \lambda^{\prime}, k$, and $\mu_{s}$. As has been discussed earlier,

TABLE III. The LSP is mostly composed of only two type of the neutral fermion fields in the basis given in Eq. (30). The other parameters are fixed as in Table I. The decay into BSM particles having branching ratios less than $0.0001 \%$ is not included here.

\begin{tabular}{|c|c|c|c|}
\hline \multirow[b]{2}{*}{ LSP type } & \multirow[b]{2}{*}{ Benchmark points } & \multicolumn{2}{|c|}{$\begin{array}{l}\text { Branching ratio of } W_{R}^{ \pm}, Z_{R} \\
\text { into different BSM fields }\end{array}$} \\
\hline & & $W_{R}^{ \pm} \rightarrow \mathrm{BSM}$ & $Z_{R} \rightarrow \mathrm{BSM}$ \\
\hline \multirow[t]{5}{*}{$\begin{array}{l}\text { 1. Bino }(49.5 \%) \text { and } \\
\text { wino } \mathrm{R}(49.5 \%)\end{array}$} & $\begin{array}{c}M_{1}=-300, M_{33,22,11}^{W_{L}}=60 \mathrm{TeV} \\
M_{33,22,11}^{W_{R}}=-2250\end{array}$ & $15.83 \%\left(W_{R}^{ \pm} \rightarrow \tilde{\chi}_{1}^{0} \tilde{\chi}_{1}^{ \pm}\right)$ & $23.34 \%\left(Z_{R} \rightarrow \tilde{\chi}_{1}^{\mp} \tilde{\chi}_{1}^{ \pm}\right)$ \\
\hline & $\mu_{s}=-12 \mathrm{TeV}, \mu_{2}=20 \mathrm{TeV}, \mu=-16 \mathrm{TeV}$ & $9.26 \%\left(W_{R}^{ \pm} \rightarrow \tilde{\chi}_{2}^{0} \tilde{\chi}_{1}^{ \pm}\right)$ & \\
\hline & $\lambda^{c}=-1.2, \lambda^{\prime}=-0.2, k=0.03$ & $0.01 \%\left(W_{R}^{ \pm} \rightarrow Z H_{1}^{ \pm}\right)$ & \\
\hline & & $0.01 \%\left(W_{R}^{ \pm} \rightarrow A_{1} W^{ \pm}\right)$ & \\
\hline & $\begin{array}{c}M_{\tilde{\chi}_{1}^{0}}=998.7 \mathrm{GeV}, M_{\tilde{\chi}_{2}^{0}}=1.681 \mathrm{TeV} \\
M_{\tilde{\chi}_{1}^{ \pm}}=984.1 \mathrm{GeV}\end{array}$ & $0.01 \%\left(W_{R}^{ \pm} \rightarrow H_{3} W^{ \pm}\right)$ & \\
\hline \multirow[t]{3}{*}{$\begin{array}{l}\text { 2. Wino L }(49.5 \%) \text { and } \\
\text { wino R }(49.5 \%)\end{array}$} & $\begin{array}{c}M_{1}=60 \mathrm{TeV}, M_{33,22,11}^{W_{L}}=502.51 \\
M_{33,22,11}^{W_{R}}=-2067.6\end{array}$ & $14.68 \%\left(W_{R}^{ \pm} \rightarrow \tilde{\chi}_{2}^{0} \tilde{\chi}_{1}^{ \pm}\right)$ & $23.35 \%\left(Z_{R} \rightarrow \tilde{\chi}_{2}^{\mp} \tilde{\chi}_{2}^{ \pm}\right)$ \\
\hline & $\begin{array}{c}\mu_{s}=-12 \mathrm{TeV}, \mu_{2}=20 \mathrm{TeV}, \mu=-16 \mathrm{TeV} \\
\lambda^{c}=-1.2, \lambda^{\prime}=-0.2, k=0.03\end{array}$ & $\begin{array}{c}12.18 \%\left(W_{R}^{ \pm} \rightarrow \tilde{\chi}_{2}^{0} \tilde{\chi}_{2}^{ \pm}\right) \\
0.01 \%\left(W_{R}^{ \pm} \rightarrow Z H_{1}^{ \pm}\right) \\
0.01 \%\left(W_{R}^{ \pm} \rightarrow A_{1} W^{ \pm}\right)\end{array}$ & \\
\hline & $\begin{array}{c}M_{\tilde{\chi}_{1}^{0}}=502.4 \mathrm{GeV}, M_{\tilde{\chi}_{2}^{0}}=526.2 \mathrm{GeV} \\
M_{\tilde{\chi}_{1}^{ \pm}}=502.5 \mathrm{GeV}\end{array}$ & $0.01 \%\left(W_{R}^{ \pm} \rightarrow H_{3} W^{ \pm}\right)$ & \\
\hline \multirow[t]{3}{*}{$\begin{array}{l}\text { 3. Wino } \mathrm{R}(49.5 \%) \text { and } \\
\text { singlino }(49.5 \%)\end{array}$} & $\begin{array}{c}M_{1}=60 \mathrm{TeV}, M_{33,22,11}^{W_{L}}=60 \mathrm{TeV} \\
M_{33,22,11}^{W_{R}}=-6500\end{array}$ & $0.1 \%\left(W_{R}^{ \pm} \rightarrow \tilde{\chi}_{1}^{0} \tilde{\chi}_{1}^{ \pm}\right)$ & $0.1 \%\left(Z_{R} \rightarrow \tilde{\chi}_{1}^{\mp} \tilde{\chi}_{1}^{ \pm}\right)$ \\
\hline & $\begin{array}{c}\mu_{s}=-5 \mathrm{TeV}, \mu_{2}=20 \mathrm{TeV}, \mu=-16 \mathrm{TeV} \\
\lambda^{c}=-1.2, \lambda^{\prime}=-0.2, k=2.4\end{array}$ & $\begin{array}{c}0.1 \%\left(W_{R}^{ \pm} \rightarrow \tilde{\chi}_{2}^{0} \tilde{\chi}_{1}^{ \pm}\right) \\
0.01 \%\left(W_{R}^{ \pm} \rightarrow Z H_{1}^{ \pm}\right) \\
0.01 \%\left(W_{R}^{ \pm} \rightarrow A_{1} W^{ \pm}\right)\end{array}$ & \\
\hline & $\begin{array}{c}M_{\tilde{\chi}_{1}^{0}}=1.66 \mathrm{TeV}, M_{\tilde{\chi}_{2}^{0}}=5.56 \mathrm{TeV} \\
M_{\tilde{\chi}_{1}^{ \pm}}=4.83 \mathrm{TeV}\end{array}$ & $0.01 \%\left(W_{R}^{ \pm} \rightarrow H_{3} W^{ \pm}\right)$ & \\
\hline
\end{tabular}


the heavy $W_{R}$ and $Z_{R}$ gauge bosons do not have significant couplings to either a bino, wino L, or singlino. Thus, the decay of these heavy gauge bosons into the light neutralinos is again almost nonexistent in these cases and is not discussed further.

\section{Combination of wino $R$ and a bino, wino $L$ or singlino}

The three benchmark points in Table III represent the cases where the LSPs are all composed of around 50\% from the $S U(2)_{R}$ wino, which does interact quite significantly with the heavy gauge bosons. BPs 1, 2, and 3 in Table III give a lightest neutralino LSP $\tilde{\chi}_{1}^{0}$ consisting of $\sim 50 \%$ wino $\mathrm{R}$ and $\sim 50 \%$ from either a bino, wino-L, or singlino field, respectively. The lightest chargino $\tilde{\chi}_{1}^{ \pm}$in BP 1 and 3 primarily consists of charged wino-R fields. Thus for these two points the decay of the $W_{R}^{ \pm} \rightarrow \tilde{\chi}_{1}^{0} \tilde{\chi}_{1}^{ \pm}$and $W_{R}^{ \pm} \rightarrow \tilde{\chi}_{2}^{0} \tilde{\chi}_{1}^{ \pm}$is quite significant with a BR around $16 \%$ and $9 \%$, respectively. The coupling strengths of the vertices $W_{R}^{ \pm} \tilde{\chi}_{1}^{0} \tilde{\chi}_{1}^{ \pm}$and $W_{R}^{ \pm} \tilde{\chi}_{2}^{0} \tilde{\chi}_{1}^{ \pm}$become almost half compared to the coupling strength of the $W_{R}^{ \pm} \tilde{\chi}_{1}^{0} \tilde{\chi}_{1}^{ \pm}$vertex in the pure wino-R case that was discussed earlier. The couplings become half due the mixing of the wino $\mathrm{R}$ with the other gaugino fields, resulting in a change of the mixing angles in $Z^{f N}$ appearing in the vertices [see Eq. (A12)] leading to $W_{R}^{ \pm}$boson decay into electroweakinos. As the combination of the lightest chargino $\tilde{\chi}_{1}^{ \pm}$remains almost similar as in the wino-R case, the $Z_{R}$ boson here decays significantly into $\tilde{\chi}_{1}^{ \pm} \tilde{\chi}_{1}^{\mp}$ with a BR of around $23.5 \%$. For BP 2 it is actually the second lightest chargino $\tilde{\chi}_{2}^{ \pm}$that is coming from the charged component of the $S U(2)_{R}$ wino. Also in this case, $W_{R}$ thus decays into $\tilde{\chi}_{2}^{ \pm} \tilde{\chi}_{1}^{0}$ and $\tilde{\chi}_{2}^{ \pm} \tilde{\chi}_{2}^{0}$ with BR of around $15 \%$ and $12 \%$, respectively, while the $Z_{R}$ boson decays into $\tilde{\chi}_{2}^{ \pm} \tilde{\chi}_{2}^{\mp}$ with a BR of around $23.5 \%$.

\section{Combination of gauginos and Higgsinos}

The only interesting cases here are the ones where the bidoublet or $S U(2)_{R}$ triplet Higgsinos mix with the wino-R state as the other cases are very similar to pure Higgsino LSP, only with reduced branchings into SUSY final states. We show these points in Table IV.

BP 1 in Table IV represents the case where the bidoublet Higgsinos mix with the wino $\mathrm{R}$ to form the lightest neutalino $\tilde{\chi}_{1}^{0}$ consisting almost $30 \%$ of each, $\tilde{\phi}_{1}^{0}, \tilde{\phi}_{2}^{0}$, and $\tilde{W}_{R}$. In order to get the LSP with almost equal contributions from the two bidoublet Higgsinos and the wino R we had to make sure that all the mass terms related to these states remained small. This in turn results in two other neutralino states remaining light. The LSP $\tilde{\chi}_{1}^{0}$ has a mass of $207.2 \mathrm{GeV}$

TABLE IV. The bidoublet or triplet Higgsinos mix with the wino R to form the lightest neutalino.

\begin{tabular}{|c|c|c|c|}
\hline \multirow[b]{2}{*}{ LSP type } & \multirow[b]{2}{*}{ Benchmark points } & \multicolumn{2}{|c|}{$\begin{array}{l}\text { Branching ratio of } W_{R}^{ \pm}, Z_{R} \\
\text { into different BSM fields }\end{array}$} \\
\hline & & $W_{R}^{ \pm} \rightarrow \mathrm{BSM}$ & $Z_{R} \rightarrow \mathrm{BSM}$ \\
\hline \multirow[t]{8}{*}{$\begin{array}{l}\text { 1. Higgsino- }\left(\phi_{1,2}\right)(36 \%, 26 \%) \\
\text { wino R }(26 \%)\end{array}$} & $\begin{array}{c}M_{1}=60 \mathrm{TeV}, M_{33,22,11}^{W_{L}}=60 \mathrm{TeV} \\
M_{33,22,11}^{W_{R}}=-2345\end{array}$ & $4.26 \%\left(W_{R}^{ \pm} \rightarrow \tilde{\chi}_{1}^{0} \tilde{\chi}_{1}^{ \pm}\right)$ & $4.19 \%\left(Z_{R} \rightarrow \tilde{\chi}_{1}^{0} \tilde{\chi}_{2}^{0}\right)$ \\
\hline & $\mu_{s}=-12 \mathrm{TeV}, \mu_{2}=20 \mathrm{TeV}, \mu=-0.2 \mathrm{TeV}$ & $3.08 \%\left(W_{R}^{ \pm} \rightarrow \tilde{\chi}_{3}^{0} \tilde{\chi}_{1}^{ \pm}\right)$ & $2.06 \%\left(Z_{R} \rightarrow \tilde{\chi}_{2}^{0} \tilde{\chi}_{3}^{0}\right)$ \\
\hline & $\lambda^{c}=-1.2, \lambda^{\prime}=-0.04, k=0.03$, & $8.06 \%\left(W_{R}^{ \pm} \rightarrow \tilde{\chi}_{1}^{0} \tilde{\chi}_{2}^{ \pm}\right)$ & $6.38 \%\left(Z_{R} \rightarrow \tilde{\chi}_{1}^{\mp} \tilde{\chi}_{1}^{ \pm}\right)$ \\
\hline & & $6.6 \%\left(W_{R}^{ \pm} \rightarrow \tilde{\chi}_{2}^{0} \tilde{\chi}_{1}^{ \pm}\right)$ & $20.15 \%\left(Z_{R} \rightarrow \tilde{\chi}_{2}^{\mp} \tilde{\chi}_{2}^{ \pm}\right)$ \\
\hline & $\begin{array}{c}M_{\tilde{\chi}_{1}^{0}}=207.2 \mathrm{GeV}, M_{\tilde{\chi}_{2}^{0}}=246.2 \mathrm{GeV} \\
M_{\tilde{\chi}_{3}^{0}}=304.2 \mathrm{GeV}\end{array}$ & $14.4 \%\left(W_{R}^{ \pm} \rightarrow \tilde{\chi}_{3}^{0} \tilde{\chi}_{2}^{ \pm}\right)$ & \\
\hline & $M_{\tilde{\chi}_{1}^{ \pm}}=237.4 \mathrm{GeV}, M_{\tilde{\chi}_{2}^{ \pm}}=1.077 \mathrm{TeV}$ & $0.01 \%\left(W_{R}^{ \pm} \rightarrow Z H_{1}^{ \pm}\right)$ & \\
\hline & & $0.01 \%\left(W_{R}^{ \pm} \rightarrow A_{1} W^{ \pm}\right)$ & \\
\hline & & $0.01 \%\left(W_{R}^{ \pm} \rightarrow H_{3} W^{ \pm}\right)$ & \\
\hline \multirow[t]{6}{*}{$\begin{array}{l}\text { 2. Higgsino- }\left(\Delta_{R}^{1,2}\right)(80 \%, 10.0 \%) \\
\text { and wino R }(6.0 \%)\end{array}$} & $\begin{array}{c}M_{1}=60 \mathrm{TeV}, M_{33,22,11}^{W_{L}}=60 \mathrm{TeV} \\
M_{33,22,11}^{W_{R}}=-2250\end{array}$ & $7.30 \%\left(W_{R}^{ \pm} \rightarrow \tilde{\chi}_{3}^{0} \tilde{\chi}_{1}^{ \pm}\right)$ & $7.77 \%\left(Z_{R} \rightarrow \tilde{\chi}_{1}^{0} \tilde{\chi}_{1}^{0}\right)$ \\
\hline & $\mu_{s}=-34, \mu_{2}=1 \mathrm{TeV}, \mu=0 \mathrm{TeV}$ & $7.30 \%\left(W_{R}^{ \pm} \rightarrow \tilde{\chi}_{2}^{0} \tilde{\chi}_{1}^{ \pm}\right)$ & $2.76 \%\left(Z_{R} \rightarrow \tilde{\chi}_{1}^{0} \tilde{\chi}_{4}^{0}\right)$ \\
\hline & $\lambda^{c}=-0.078, \lambda^{\prime}=-1.7, k=0.03$ & $6.44 \%\left(W_{R}^{ \pm} \rightarrow \tilde{\chi}_{1}^{0} \tilde{\chi}_{2}^{ \pm}\right)$ & $5.83 \%\left(Z_{R} \rightarrow \tilde{\chi}_{2}^{0} \tilde{\chi}_{3}^{0}\right)$ \\
\hline & & $0.3 \%\left(W_{R}^{ \pm} \rightarrow \tilde{\chi}_{3}^{0} \tilde{\chi}_{2}^{ \pm}\right)$ & $5.81 \%\left(Z_{R} \rightarrow \tilde{\chi}_{1}^{ \pm} \tilde{\chi}_{1}^{\mp}\right)$ \\
\hline & $\begin{array}{c}M_{\tilde{\chi}_{1}^{0}}=761.6 \mathrm{GeV}, M_{\tilde{\chi}_{2}^{0}}=1.69 \mathrm{TeV}, \\
M_{\tilde{\chi}_{3}^{0}}=1.70 \mathrm{TeV}\end{array}$ & $6.90 \%\left(W_{R}^{ \pm} \rightarrow \tilde{\chi}^{ \pm \pm} \tilde{\chi}_{1}^{\mp}\right)$ & $11.01 \%\left(Z_{R} \rightarrow \tilde{\chi}^{ \pm \pm} \tilde{\chi}^{\mp \mp}\right)$ \\
\hline & $M_{\tilde{\chi}_{1}^{ \pm}}=1.69 \mathrm{TeV}, M_{\tilde{\chi}_{2}^{ \pm}}=2.77 \mathrm{GeV}$ & $\begin{array}{l}0.01 \%\left(W_{R}^{ \pm} \rightarrow Z H_{1}^{ \pm}\right) \\
0.01 \%\left(W_{R}^{ \pm} \rightarrow A_{2} W^{ \pm}\right) \\
0.01 \%\left(W_{P}^{ \pm} \rightarrow H_{3} W^{ \pm}\right)\end{array}$ & \\
\hline
\end{tabular}


for our chosen parameters. The next lightest neutralino $\tilde{\chi}_{2}^{0}$ is mostly coming from the bidoublet fields and has a mass of $246.2 \mathrm{GeV}$. The next heavier member in the neutralino spectrum $\tilde{\chi}_{3}^{0}$ gets a large contribution from the wino-R field and has a mass of $304.2 \mathrm{GeV}$. The chargino $\tilde{\chi}_{2}^{ \pm}$primarily consists of a charged component of wino-R field, whereas $\tilde{\chi}_{1}^{\mp}$ consist of bidoublet fields. They have masses in the same order as the neutralino masses as expected. Thus a number of different channels open up for the $W_{R}$ and $Z_{R}$ decay albeit with smaller branching ratios compared to the pure Higgsino or pure wino-R case. However, the total branching into final state BSM particles becomes large since a host of new decay channels have now opened up. The largest non-SM decay of $W_{R}$ is in the $W_{R}^{ \pm} \rightarrow \tilde{\chi}_{3}^{0} \tilde{\chi}_{2}^{ \pm}$ channel with $14.4 \%$ BR (much smaller than the pure wino$\mathrm{R}$ case with $\mathrm{BR}$ of $26.8 \%$ ) due to the mixing suppression in the neutralino and the chargino sectors. Similarly the $W_{R}^{ \pm} \rightarrow \tilde{\chi}_{1,2}^{0} \tilde{\chi}_{1}^{ \pm}$channels are smaller here as can be seen in Table IV. A few other new combinations of neutralino plus chargino final states open up due to the smaller mass of the electroweakinos in this case. We find the total branching of $W_{R}$ into BSM final state particles to be roughly $36.6 \%$. Notably the $W_{R}^{ \pm} \rightarrow \tilde{\chi}_{2}^{0} \tilde{\chi}_{2}^{ \pm}$channel is very small since there is no direct $W_{R}^{ \pm} \tilde{W}_{R}^{\mp} \tilde{\Phi}$ coupling in the gauge basis and only arises from mixings that are quite small. Similarly $Z_{R}$ has a number of possible decay channels including $Z_{R} \rightarrow \tilde{\chi}_{1}^{0} \tilde{\chi}_{2}^{0}$, $Z_{R} \rightarrow \tilde{\chi}_{2}^{0} \tilde{\chi}_{3}^{0}, Z_{R} \rightarrow \tilde{\chi}_{1}^{ \pm} \tilde{\chi}_{1}^{\mp}$, and $Z_{R} \rightarrow \tilde{\chi}_{2}^{ \pm} \tilde{\chi}_{2}^{\mp}$ with $4.2 \%$, $2.1 \%, 6.4 \%$, and $20.15 \%$ BR in each channel, respectively. The coupling strength of the vertex $Z_{R} \tilde{\chi}_{2}^{ \pm} \tilde{\chi}_{2}^{ \pm}$is again slightly smaller than the pure wino-R (here $\tilde{\chi}_{2}^{ \pm}$is wino$\mathrm{R}$ dominated) case due to its mixing with Higgsino states in this case.

One can adjust the parameters $M_{33}^{W_{R}}, \lambda^{\prime}, \lambda^{c}$, and $\mu_{2}$ to get neutralino LSP that consists of neutral fermionic components wino R and two Higgsino from triplet fields. In this case, it is not possible to get $33 \%$ contribution to the LSP from each of the fields due to the large off-diagonal $\left(g_{V} v_{R}\right.$, $g_{R} v_{R}, g_{V} \bar{v}_{R}$, and $g_{R} \bar{v}_{R}$ ) terms in the neutralino mass matrix [see Eq. (31)]. We represent this as BP 2 and the corresponding contributions of each of these fields are shown in Table IV. Similar to the previous case with light triplet, the doubly charged chargino remains light here as well. We get the light neutralino and chargino particles masses as $M_{\tilde{\chi}_{1}^{0}}=761.6 \mathrm{GeV}, M_{\tilde{\chi}_{2}^{0}}=1.69 \mathrm{TeV}$, $M_{\tilde{\chi}_{3}^{0}}=1.7 \mathrm{TeV}, \quad M_{\tilde{\chi}_{1}^{ \pm}}=1.69 \mathrm{TeV}, \quad M_{\tilde{\chi}_{2}^{ \pm}}=2.77, \quad$ and $M_{\tilde{\chi}^{ \pm \pm}}=0.922 \mathrm{TeV}$ for this choice of BP. The $\tilde{\chi}_{1}^{0}$ and $\tilde{\chi}_{2}^{0}$ are both coming from a combination of the triplet and the wino-R fields. The lightest chargino $\tilde{\chi}_{1}^{ \pm}$has around $90 \%$ Higgsino components from the triplet and $6 \%$ from $S U(2)_{R}$ wino component. The decay channels for the $W_{R}$ and the $Z_{R}$ fields are given in BP2 of Table IV. As the $\tilde{\chi}_{3}^{0}, \tilde{\chi}_{2}^{ \pm}$, and $\tilde{\chi}^{ \pm \pm}$are mostly coming from the triplet sector, the corresponding decay of the heavy gauge bosons into these particles remains large. The partial decay widths (and hence the branching ratios), though, are slightly smaller compared to the case with the pure triplet Higgsino LSP due to larger mixing with the $S U(2)_{R}$ wino.

\section{Combination of bidoublet and triplet Higgsinos}

We discuss a parameter choice where the contribution to the LSP is mainly coming from the mixing between the bidoublet and right-handed triplet Higgsino fields. In this case, we get four light neutralinos having masses $M_{\tilde{\chi}_{1}^{0}}=236 \mathrm{GeV}, \quad M_{\tilde{\chi}_{2}^{0}}=240 \mathrm{GeV}, \quad M_{\tilde{\chi}_{3}^{0}}=246.5 \mathrm{GeV}$, and $M_{\tilde{\chi}_{4}^{0}}=4.25 \mathrm{TeV}$. Here $\tilde{\chi}_{1}^{0}$ and $\tilde{\chi}_{3}^{0}$ are composed of $25 \%$ from each of the Higgsino fields; $\tilde{\chi}_{2}^{0}$ is almost entirely coming from the bidoublet field while $\tilde{\chi}_{4}^{0}$ is from the triplet fields. The lightest chargino mainly has charged bidoublet Higgsino contribution with $M_{\tilde{\chi}_{1}^{ \pm}}=240 \mathrm{GeV}$ and the second lightest chargino state is mainly from the triplet Higgsino with mass $M_{\tilde{\chi}_{2}^{ \pm}}=1.77 \mathrm{TeV}$. The light doubly charged chargino arising from the $S U(2)_{R}$ triplet Higgsino has $M_{\tilde{\chi}^{ \pm \pm}}=2.16 \mathrm{TeV}$.

A host of new decay channels for the heavy gauge bosons decaying into these light electroweakinos are obtained here. The $W_{R}^{ \pm} \tilde{\chi}_{i}^{0} \tilde{\chi}_{j}^{\mp}$ and $W_{R}^{ \pm} \tilde{\chi}^{\mp \mp} \tilde{\chi}_{j}^{ \pm}$couplings are given in Eqs. (A12) and (A13) while the $Z_{R} \tilde{\chi}_{i}^{0} \tilde{\chi}_{j}^{0}, Z_{R} \tilde{\chi}_{i}^{ \pm} \tilde{\chi}_{j}^{\mp}$, and $Z_{R} \tilde{\chi}^{ \pm \pm} \tilde{\chi}^{\mp \mp}$ are presented in Eqs. (B16)-(B18). Since the gauge bosons couple more strongly with the triplets compared to the bidoublets, the branching ratio of $\tilde{\chi}^{ \pm \pm} \tilde{\chi}_{2}^{ \pm}$is quite large of the order of $8.29 \%$, respectively. As stated earlier, $\tilde{\chi}_{2}^{ \pm}$and $\tilde{\chi}^{ \pm \pm}$are mostly coming from the triplet fields. However, $W_{R}$ into $\tilde{\chi}_{4}^{0} \tilde{\chi}_{2}^{ \pm}$are negligible due to the mass of the final states. Similarly $Z_{R}$ decays involving $\tilde{\chi}_{4}^{0}$ in the final state are much larger than the others. All the important BSM decay channels of the heavy gauge bosons for this case are given in Table V. Combining all these channels, the total branching ratios of the $W_{R}$ and $Z_{R}$ bosons into BSM final states become $49.93 \%$ and $50.54 \%$, respectively, which are larger than the scenarios with almost pure bidoublet or triplet Higgsino-like LSP.

\section{Case-III: Pure LSP and a light pair of squarks}

In the previous sections, the squarks and sleptons masses were larger than the $W_{R}$ and $Z_{R}$ masses so that their decays into final state squarks and sleptons were kinematically disallowed. We now study the effect of low mass sfermions on the possible decays of the heavy gauge bosons. In order to achieve this, we significantly decrease the values of the soft masses $M_{q L}$ and $M_{q R}$ for the squarks and sleptons so that they are light enough for the heavy gauge bosons to decay into these particles. The small top squark mass significantly affects the Higgs boson loop-corrected mass and we have to adjust the parameters $M_{q L}$ and $M_{q R}\left(T_{33}^{y q}\right.$ remains small to avoid the color breaking minima along 
TABLE V. Combination of bidoublet and triplet Higgsinos: The LSP are composed of $25 \%$ from each of the Higgsino fields.

\begin{tabular}{lccc}
\hline \hline LSP type & Benchmark point & \multicolumn{3}{c}{ Branching ratio of $W_{R}^{ \pm}, Z_{R}$ into different BSM fields } \\
\cline { 2 - 4 } $\begin{array}{l}\text { 1. Higgsino- }\left(\phi_{1,2} \text { and } \Delta_{R}^{1,2}\right) \\
(\sim 25 \% \text { each })\end{array}$ & $M_{1}=60 \mathrm{TeV}, M_{33,22,11}^{W_{L}}=60 \mathrm{TeV}$, & $4.98 \%\left(W_{R}^{ \pm} \rightarrow \tilde{\chi}_{1}^{0} \tilde{\chi}_{1}^{ \pm}\right)$ & $4.8 \%\left(Z_{R} \rightarrow \tilde{\chi}_{1}^{0} \tilde{\chi}_{2}^{0}\right)$ \\
& $M_{33,22,11}^{W_{R}}=60 \mathrm{TeV}$, & \\
$\mu_{s}=-12 \mathrm{TeV}, \mu_{2}=-1 \mathrm{TeV}, \mu=-0.2 \mathrm{TeV}$, & $7.21 \%\left(W_{R}^{ \pm} \rightarrow \tilde{\chi}_{0}^{0} \tilde{\chi}_{1}^{ \pm}\right)$ & $2.28 \%\left(Z_{R} \rightarrow \tilde{\chi}_{2}^{0} \tilde{\chi}_{3}^{0}\right)$ \\
$\lambda^{c}=-1.16, \lambda^{\prime}=-0.04, k=0.03$, & $2.62 \%\left(W_{R}^{ \pm} \rightarrow \tilde{\chi}_{3}^{0} \tilde{\chi}_{1}^{ \pm}\right)$ & $5.40 \%\left(Z_{R} \rightarrow \tilde{\chi}_{3}^{0} \tilde{\chi}_{3}^{0}\right)$ \\
& $4.31 \%\left(W_{R}^{ \pm} \rightarrow \tilde{\chi}_{1}^{0} \tilde{\chi}_{2}^{ \pm}\right)$ & $1.59 \%\left(Z_{R} \rightarrow \tilde{\chi}_{3}^{0} \tilde{\chi}_{4}^{0}\right)$ \\
& $7.5 \%\left(W_{R}^{ \pm} \rightarrow \tilde{\chi}_{3}^{0} \tilde{\chi}_{2}^{ \pm}\right)$ & $5.66 \%\left(Z_{R} \rightarrow \tilde{\chi}_{1}^{\mp} \tilde{\chi}_{1}^{ \pm}\right)$ \\
& $8.29 \%\left(W_{R}^{ \pm} \rightarrow \tilde{\chi}^{ \pm \pm} \tilde{\chi}_{2}^{\mp}\right)$ & $2.09 \%\left(Z_{R} \rightarrow \tilde{\chi}_{2}^{\mp} \tilde{\chi}_{2}^{ \pm}\right)$ \\
$M_{\tilde{\chi}_{1}^{0}}=236 \mathrm{GeV}, M_{\tilde{\chi}_{2}^{0}}=240 \mathrm{GeV}$, & $0.01 \%\left(W_{R}^{ \pm} \rightarrow H_{3} W^{ \pm}\right)$ & $10.01 \%\left(Z_{R} \rightarrow \tilde{\chi}^{\mp} \tilde{\chi}^{ \pm \pm}\right)$ \\
$M_{\tilde{\chi}_{3}^{0}}=246.5 \mathrm{GeV}$ & & \\
$M_{\tilde{\chi}_{1}^{ \pm}}=240 \mathrm{GeV}, M_{\tilde{\chi}_{2}^{ \pm}}=1.74 \mathrm{TeV}$ & $0.01 \%\left(W_{R}^{ \pm} \rightarrow Z H_{1}^{ \pm}\right)$ & $0.01 \%\left(Z_{R} \rightarrow H_{2} A_{1}\right)$ \\
\hline \hline
\end{tabular}

the squark-field directions [34-36]) to account for this change. One has to choose a reasonably large $T^{y q}$ for this, resulting in the left-handed squarks becoming quite massive (more than $M_{W_{R}} / 2$ ). The right-handed squarks though can remain light since the corrections to the right-handed squarks coming from the triplet VEV through the D-term are negative for our choice of parameters. Thus the heavy gauge bosons can decay into these right-handed squark final states.

We change the values of the soft mass-squared terms $M_{l L}^{2}, M_{l R}^{2}, M_{q L}^{2}$, and $M_{q R}^{2}$ to get lighter $(\mathcal{O}(1) \mathrm{TeV})$ squarks and sleptons. Therefore the heavy vector boson decays into final state squarks and sleptons are kinematically allowed. It must be noted here that the heavy sneutrino masses have not been altered and hence sneutrino final states are still absent. The relevant heavy gauge boson couplings with the squarks and sleptons are given in Eqs. (A2), (B4), (B7), and (B5). It is easy to see that these coupling strengths are proportional to the momentum of the final state particles. Hence the decay widths significantly depend on the momentum of the final state particles, which are

$$
\left|\vec{p}_{c m}\right|=\frac{1}{2 M_{V}} \sqrt{M_{V}^{4}+M_{\tilde{q}_{i}}^{4}+M_{\tilde{q}_{i}}^{4}-2 M_{V}^{2} M_{\tilde{q}_{i}}^{2}-2 M_{V}^{2} M_{\tilde{q}_{j}}^{2}-2 M_{\tilde{q}_{i}}^{2} M_{\tilde{q}_{j}}^{2}}
$$

Here $M_{V}$ is the gauge boson mass and $M_{\tilde{q}_{i, j}}$ are the squark or slepton masses. The masses of the light up-type squarks (primarily consisting of right-handed squark fields) remain always larger than the light down-type squarks (also right-handed) for our choice of parameters due to the opposite sign contribution from the $S U(2)_{R}$ D-term. This term is additive for the $m_{\tilde{u}_{R} \tilde{u}_{R}^{*}}$ term while it is subtractive for the $m_{\tilde{d}_{R}} \tilde{d}_{R}^{*}$.

Let us consider the benchmark point where we have a binolike, $S U(2)_{L}$ winolike, and singlinolike LSP. We keep the numerical values of all the parameters the same except we now choose $M_{q L, i i}^{2}=M_{q R, i i}^{2}=16 \mathrm{TeV}^{2}(i=1,2)$, $M_{q L, 33}^{2}=16$ and $M_{q R, 33}^{2}=12 \mathrm{TeV}^{2}$, and $M_{l L, i i}^{2}=M_{l R, i i}^{2}=$ $9.6 \mathrm{TeV}^{2}(i=1,2,3)$ to get the lightest down-type squark mass $m_{\tilde{d}_{1}}=1 \mathrm{TeV}$ while the other two are $m_{\tilde{d}_{2,3}}=2.6 \mathrm{TeV}$. The lightest up-type squark mass $m_{\tilde{u}_{1}}=2.2 \mathrm{TeV}$ while $m_{\tilde{u}_{2,3}}=4.1 \mathrm{TeV}$. The charged slepton masses are $m_{\tilde{e}_{1}}=$ $2.02 \mathrm{TeV}, m_{\tilde{e}_{2}}=2.02 \mathrm{TeV}$, and $m_{\tilde{e}_{3}}=2.5 \mathrm{TeV}$. These relatively light squarks and sleptons are mostly composed of the right-handed fields. All other squark and slepton masses remain heavier than $3.0 \mathrm{TeV}$. Hence the $W_{R}$ boson decays into $\tilde{u}_{i}$ plus $\tilde{d}_{i}(i=1,2,3)$ final state particles become accessible while slepton final states are still forbidden owing to the large sneutrino masses. We find that the decay channels of the $W_{R}$ boson into squarks $\left(W_{R}^{ \pm} \rightarrow \tilde{q}_{i} \tilde{q}_{j}{ }^{*}\right)$ each has a BR of around $0.1 \%$ due to the mass and $W_{R}^{ \pm} \tilde{q}_{i} \tilde{q}_{j}$ coupling strength. The $Z_{R}$ boson, on the other hand, can decay into the final states with $\tilde{u}_{i} \tilde{u}_{i}^{*}, \tilde{d}_{i} \tilde{d}_{i}^{*}$, or $\tilde{e}_{i} \tilde{e}_{i}^{*}(i=1,2,3)$ particles. We get roughly $\sim 10 \%$ branching in the final state $\tilde{d}_{i} \tilde{d}_{i}^{*}$ channels while the final state $\tilde{u}_{i} \tilde{u}_{i}^{*}, \tilde{e}_{i} \tilde{e}_{i}^{*}$ decay channels are suppressed due to their small couplings and larger masses. We show the total branching ratio into the final state squarks in Table VII. Also in this case, the masses of the neutralinos and charginos remain almost the same as case 1 in Table II. The $W_{R}$ and $Z_{R}$ boson decays into a combination of $\tilde{\chi}_{1}^{0}$ 
TABLE VI. The remaining parameters are fixed as in Table I. The electroweakino masses remain the same as the single component LSP in Table II.

\begin{tabular}{|c|c|c|}
\hline \multirow[b]{2}{*}{ LSP type } & \multicolumn{2}{|c|}{$\begin{array}{c}\text { Branching ratio of } W_{R}^{ \pm}, Z_{R} \text { into different BSM fields for the common choice of } \\
M_{q L, i i}^{2}=M_{q R, i i}^{2}=16 \mathrm{TeV}^{2}(i=1,2) \text { and } M_{q L, 33}^{2}=4 \mathrm{TeV}^{2} \text { and } M_{q R, 33}^{2}=12 \mathrm{TeV}^{2}\end{array}$} \\
\hline & $W_{R}^{ \pm} \rightarrow \mathrm{BSM}$ & $Z_{R} \rightarrow \mathrm{BSM}$ \\
\hline Bino, wino L, singlino & $\begin{array}{c}<1 \%\left(W_{R}^{ \pm} \rightarrow \sum \tilde{u}_{i} \tilde{d}_{i}\right) \\
0.1 \%\left(W_{R}^{ \pm} \rightarrow Z H_{1}^{ \pm}\right) \\
0.1 \%\left(W_{R}^{ \pm} \rightarrow A_{1} W^{ \pm}\right) \\
0.1 \%\left(W_{R}^{ \pm} \rightarrow H_{3} W^{ \pm}\right)\end{array}$ & $\begin{array}{l}10 \%\left(Z_{R} \rightarrow \sum \tilde{d}_{i} \tilde{d}_{i}\right) \\
<1 \%\left(Z_{R} \rightarrow \sum \tilde{u}_{i} \tilde{u}_{i}\right)\end{array}$ \\
\hline Wino R (3b) & $\begin{array}{l}26.80 \%\left(W_{R}^{ \pm} \rightarrow \tilde{\chi}_{1}^{0} \tilde{\chi}_{1}^{ \pm}\right) \\
<1 \%\left(W_{R}^{ \pm} \rightarrow \sum \tilde{u}_{i} \tilde{d}_{i}\right) \\
0.01 \%\left(W_{R}^{ \pm} \rightarrow Z H_{1}^{ \pm}\right) \\
0.01 \%\left(W_{R}^{ \pm} \rightarrow A_{1} W^{ \pm}\right) \\
0.01 \%\left(W_{R}^{ \pm} \rightarrow H_{3} W^{ \pm}\right)\end{array}$ & $\begin{array}{l}21.01 \%\left(Z_{R} \rightarrow \tilde{\chi}_{1}^{\mp} \tilde{\chi}_{1}^{ \pm}\right) \\
9 \%\left(Z_{R} \rightarrow \sum \tilde{d}_{i} \tilde{d}_{i}\right) \\
<1 \%\left(Z_{R} \rightarrow \sum \tilde{u}_{i} \tilde{u}_{i}\right)\end{array}$ \\
\hline Higgsino- $\left(\phi_{1,2}\right)$ & $\begin{array}{l}9.03 \%\left(W_{R}^{ \pm} \rightarrow \tilde{\chi}_{1}^{0} \tilde{\chi}_{1}^{ \pm}\right) \\
9.03 \%\left(W_{R}^{ \pm} \rightarrow \tilde{\chi}_{2}^{0} \tilde{\chi}_{1}^{ \pm}\right) \\
<1 \%\left(W_{R}^{ \pm} \rightarrow \sum \tilde{u}_{i} \tilde{d}_{i}\right) \\
0.01 \%\left(W_{R}^{ \pm} \rightarrow Z H_{1}^{ \pm}\right) \\
0.01 \%\left(W_{R}^{ \pm} \rightarrow A_{1} W^{ \pm}\right) \\
0.01 \%\left(W_{R}^{ \pm} \rightarrow H_{3} W^{ \pm}\right)\end{array}$ & $\begin{array}{l}7.07 \%\left(Z_{R} \rightarrow \tilde{\chi}_{1}^{0} \tilde{\chi}_{2}^{0}\right) \\
7.07 \%\left(Z_{R} \rightarrow \tilde{\chi}_{1}^{\mp} \tilde{\chi}_{1}^{ \pm}\right) \\
14 \%\left(Z_{R} \rightarrow \sum \tilde{d}_{i} \tilde{d}_{i}\right) \\
<1 \%\left(Z_{R} \rightarrow \sum \tilde{u}_{i} \tilde{u}_{i}\right)\end{array}$ \\
\hline $\operatorname{Higgsino}-\left(\Delta_{R}^{1,2}\right)$ & $\begin{array}{c}10.37 \%\left(W_{R}^{ \pm} \rightarrow \tilde{\chi}_{1}^{0} \tilde{\chi}_{1}^{ \pm}\right) \\
10.35 \%\left(W_{R}^{ \pm} \rightarrow \tilde{\chi}_{2}^{0} \tilde{\chi}_{1}^{ \pm}\right) \\
20.84 \%\left(W_{R}^{p} m \rightarrow \tilde{\chi}_{1}^{\mp} \tilde{\chi}^{ \pm \pm}\right) \\
<1 \%\left(W_{R}^{ \pm} \rightarrow \sum \tilde{u}_{i} \tilde{d}_{i}\right) \\
0.01 \%\left(W_{R}^{ \pm} \rightarrow Z H_{1}^{ \pm}\right) \\
0.01 \%\left(W_{R}^{ \pm} \rightarrow A_{2} W^{ \pm}\right) \\
0.01 \%\left(W_{R}^{ \pm} \rightarrow H_{3} W^{ \pm}\right)\end{array}$ & $\begin{array}{c}29.01 \%\left(Z_{R} \rightarrow \tilde{\chi}_{1}^{0} \tilde{\chi}_{2}^{0}\right) \\
1.61 \%\left(Z_{R} \rightarrow \tilde{\chi}_{1}^{\mp} \tilde{\chi}_{1}^{ \pm}\right) \\
8.04 \%\left(Z_{R} \rightarrow \tilde{\chi}^{\mp \mp} \tilde{\chi}^{ \pm \mp}\right) \\
6 \%\left(Z_{R} \rightarrow \sum \tilde{d}_{i} \tilde{d}_{i}\right) \\
<1 \%\left(Z_{R} \rightarrow \sum \tilde{u}_{i} \tilde{u}_{i}\right)\end{array}$ \\
\hline
\end{tabular}

and $\tilde{\chi}_{1}^{ \pm}$final states remain negligibly small as discussed before. Thus the only BSM decays of the heavy gauge bosons are into the light squarks in this case.

The left-handed winolike LSP primarily consists of $\tilde{W}_{L}^{0}$ gauge boson fields. We use similar numerical values of the parameters as in the previous case to get the lightest down-type squark with $m_{\tilde{d}_{1}}=1 \mathrm{TeV}$. The decay width and branching of the heavy vector bosons $W_{R}$ and $Z_{R}$ into the final state squarks remain almost similar as in the previous binolike LSP case.

The choice of BP 1(a) and 1(b) in Table II [with $S U(2)_{R}$ winolike LSP] can also admit light squarks by choosing appropriate values of $M_{q L, i i}^{2}$ and $M_{q R, i i}^{2}$. These give almost similar decay width of the heavy vector bosons $W_{R}$ and $Z_{R}$ into the final state squarks as discussed above. As a result, the branching of $W_{R}^{ \pm} \rightarrow \tilde{\chi}_{1}^{0} \tilde{\chi}_{1}^{ \pm}$remains the same while the $Z_{R} \rightarrow \tilde{\chi}_{1}^{ \pm} \tilde{\chi}_{1}^{\mp}$ channel decreases by $\sim 3 \%$.

Since $\lambda^{c}, \mu_{2}$, and $\mu$ have different numerical values for the choice of BP 2 and 3, we use the same $M_{q L, i i}^{2}$ and $M_{q R, i i}^{2}$ to get the lightest squark mass $m_{\tilde{d}_{1}}=1 \mathrm{TeV}$. The Higgslike scalar mass remain $125 \mathrm{GeV}$. Also in these cases, the decay width into new final state squark channels remains almost the same and hence the branching ratios of both the SM and BSM, i.e., combination of neutralinos and/or charginos final state, have changed as can be seen in Table VI.

\section{COLLIDER SIGNATURE OF THE HEAVY GAUGE BOSONS}

The decay modes of the heavy gauge bosons into pairs of leptons or jets can be considered a direct probe of these heavy gauge bosons in the context of collider searches. It has already been studied in the literature for various kinds of models with extra gauge bosons [12,16-18]. Multilepton final states arising through $W_{R}$ mediated processes have also been considered in the context of dark matter studies in LRSUSY models [37,38]. The heavy gauge bosons in our model can also have significant branching ratios into final states involving quarks and charged leptons. Processes involving right-handed neutrino final states though are absent here due to these particles being heavier than the heavy gauge bosons. Hence, similar to the previous studies, 
TABLE VII. The branching fraction of relevant processes for these benchmark points to analyze the mono- $X(X=W, Z)$ plus $\mathbb{E}_{T}$ and dilepton signatures through the cascade and direct decay of the heavy gauge bosons. The other input parameters and the heavy gauge bosons masses are fixed as in the previous section.

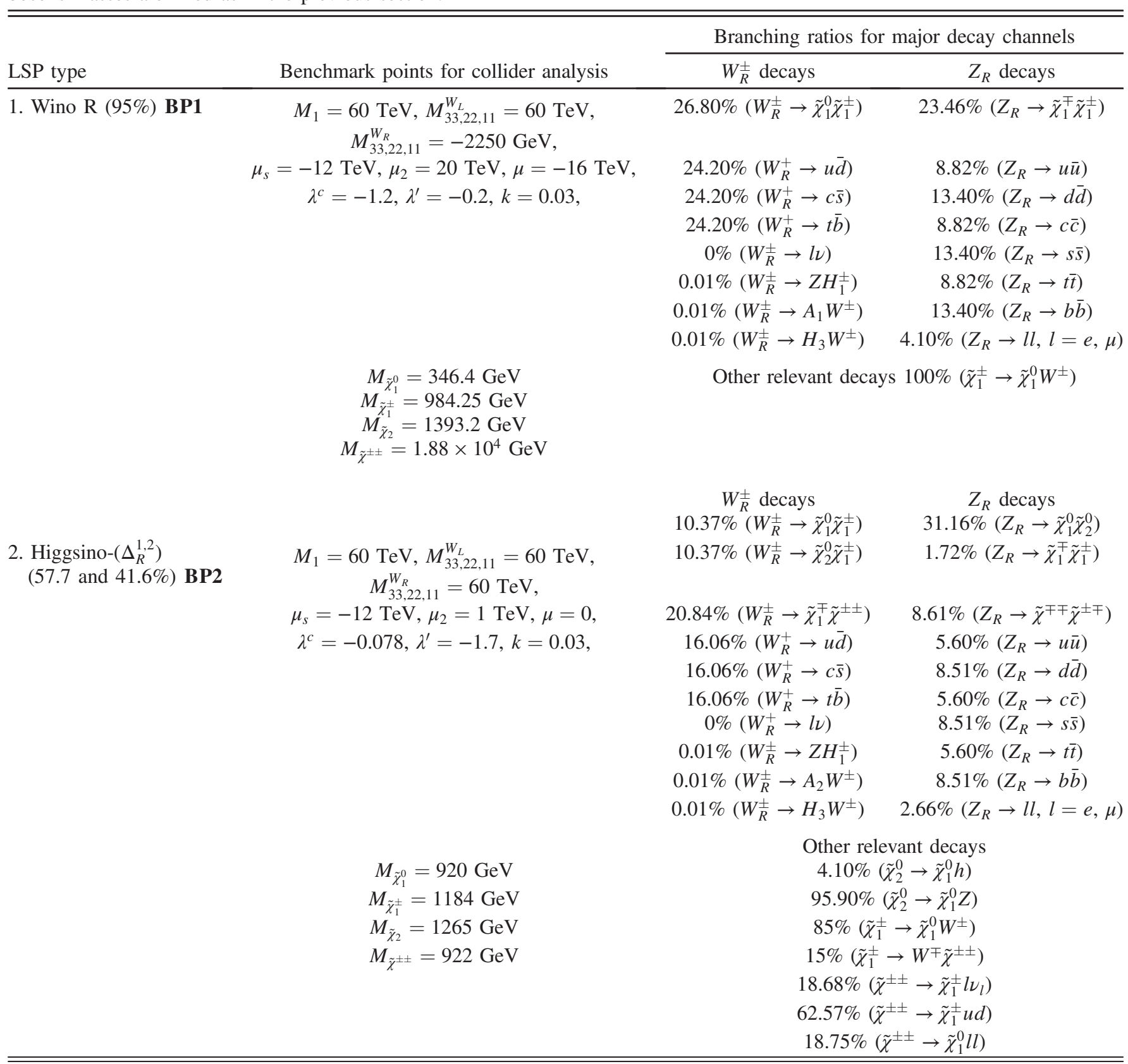

one can probe the gauge bosons in the LRSUSY model in the dilepton and dijet final states. However, in the presence of light supersymmetric particles, various other decay channels with significant branching are also present. It would be interesting to study these new channels involving the light electroweakinos as they may reveal interesting features of this model in the context of collider searches. These may even be helpful in suggesting new direction to search for these extra gauge bosons. To address these questions, we analyze several possible decay channels for the heavy gauge bosons in the context of the HL-LHC with $\sqrt{s}=14 \mathrm{TeV}$ energy and a luminosity of $3000 \mathrm{fb}^{-1}$ and the HE-LHC with $27 \mathrm{TeV}$ CoM energy and luminosity of $3000 \mathrm{fb}^{-1}$. Before we discuss the new SUSY decay channels, we first analyze the familiar dilepton and dijet final states arising from the decay of the heavy gauge bosons in our model.

We consider two benchmark points (BP1 and BP2) in Table VII corresponding to two distinctly different compositions for the LSP. In both of these two BPs, the electroweakino masses are taken around $\mathrm{a} \mathrm{TeV}$ so that 


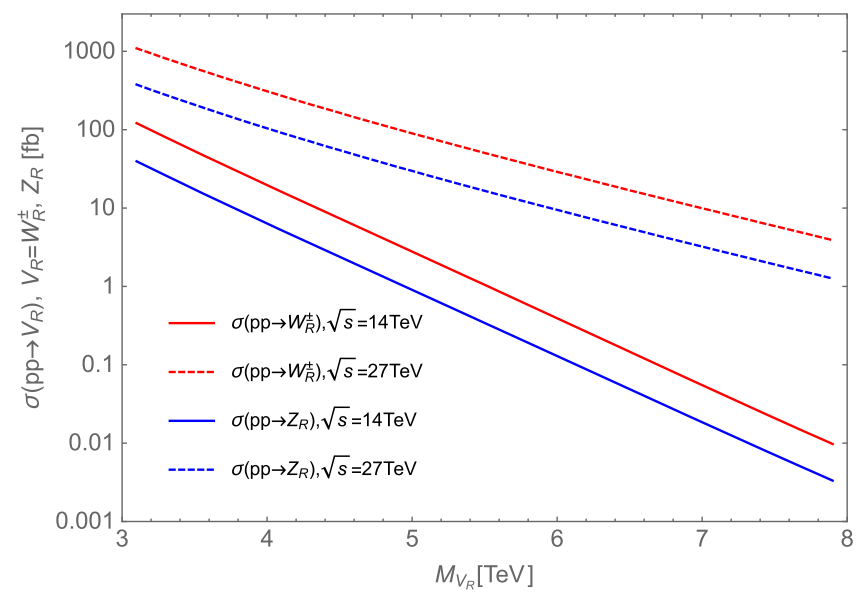

FIG. 1. Production cross section for the heavy $W_{R}$ and $Z_{R}$ bosons at the 14 and $27 \mathrm{TeV}$ LHC experiment.

one could avoid the present experimental constraints. The BPs are chosen such that the heavy gauge bosons have significant decay BRs into SUSY final states as they are also used later in our analysis of SUSY decays of $W_{R}$ and $Z_{R}$ bosons.

The gauge bosons are produced via the s-channel quarkquark scattering processes. The production cross sections of the heavy gauge boson as a function of their masses are shown in Fig. 1 for the 14 and $27 \mathrm{TeV}$ c.m. energies at the LHC. We also list the respective production cross sections for gauge bosons masses of $M_{W_{R}}=5.25 \mathrm{TeV}$ and $M_{Z_{R}}=$ 8.5 TeV in Table VIII, as those are the ones we consider extensively for our analysis. We use SARAH-4.8.6 $[39,40]$ to get the input codes for SPheno-4.0.3 [41,42] and MadGraph-2.3.3 [43]. The SPheno software helps to produce the particle spectrum. Using this spectrum in the MadGraph-2.3.3, we calculate the production cross section of the heavy gauge bosons. We use MadGraph-2.3.3 to generate the signal as well as background events and PYTHIA-6.4.28 [44] for showering and hadronization. All generated signal and background events are processed through a fast simulation package Delphes-3.4.1 [45] and we choose the ALTAS configuration card for the analysis.

\section{A. Dilepton searches}

The major contributions to the dilepton final states from the heavy gauge bosons in this model are coming from the

TABLE VIII. Production cross sections of the heavy gauge bosons with mass $M_{W_{R}}=5.25 \mathrm{TeV}$ and $M_{Z_{R}}=8.5 \mathrm{TeV}$ at $\sqrt{s}=14$ and $27 \mathrm{TeV}$, respectively.

\begin{tabular}{lccc}
\hline \hline & & \multicolumn{2}{c}{ Cross section in $[\mathrm{fb}]$} \\
\cline { 3 - 4 } Sl. no. & Processes & $14 \mathrm{TeV}$ & $27 \mathrm{TeV}$ \\
\hline 1 & $p p \rightarrow W_{R}^{ \pm}$ & 1.72 & 67.39 \\
2 & $p p \rightarrow Z_{R}$ & $1.05 \times 10^{-3}$ & 0.673 \\
\hline \hline
\end{tabular}

$Z_{R}$ bosons decaying into the same-flavor opposite-sign (SFOS) leptonic final states with BRs of $4.04 \%$ and $2.66 \%$ for BP1 and BP2, respectively. The charged gauge boson $W_{R}$ decaying into leptonic final states $W_{R}^{ \pm} \rightarrow l \nu, l=e, \mu$ is negligibly small due to the heavier right-handed neutrinos as was discussed earlier. We thus analyze the dilepton (electron or muon) final state signals at the 14 and $27 \mathrm{TeV}$ LHC to investigate the possibility of identifying possible dilepton signal from the $Z_{R}$ decays in this model. The events are selected with each isolated lepton (electron or muon) having transverse momentum $p_{T}$ larger than $30 \mathrm{GeV}$. Also the candidate electron (muon) is required to satisfy a rapidity cut $|\eta|<2.5$.

Several SM processes can contribute as background for the dilepton signal arising from the decay of the $Z_{R}$ boson. Among them, the $p p \rightarrow Z / \gamma^{*} \rightarrow l l$ channel becomes dominant due to the presence of virtual photon mediated processes. The other processes like $p p \rightarrow Z Z(Z \rightarrow l l$, $Z \rightarrow \nu \bar{\nu}), p p \rightarrow Z W(Z \rightarrow l l, W \rightarrow l \nu), p p \rightarrow W W(W \rightarrow l \nu)$, and $p p \rightarrow t \bar{t}(t \rightarrow W b)$ also add to the SM background. Invariant mass distribution for the signal and background events are shown in Fig. 2 for LHC energy of $\sqrt{s}=$ $14 \mathrm{TeV}$ (left) and $\sqrt{s}=27 \mathrm{TeV}$ (right). One can understand the shape of the invariant mass distribution as a consequence of the lepton smearing and final state radiation.

In our analysis, a significant contribution to the dilepton background events can also arise from the $p p \rightarrow j j$ where the jets $j$ can fake as leptons $(0.5 \%$ into electron, and $0.1 \%$ into muon). In fact, the jets faking lepton background for $p_{T}^{j}>20 \mathrm{GeV}$ become larger than the $p p \rightarrow Z / \gamma^{*} \rightarrow l l$ events due to their large production cross section. It could effectively be reduced by the large $p_{T}^{j}$ cut on the selected background events. The signal events $p p \rightarrow Z_{R} \rightarrow l l$ though are also affected by the same $p_{T}^{l}$ cut. We have thus selected only the signal as well as the background events with $p_{T}^{j, l}>1 \mathrm{TeV}$. The signal region $8.0<M_{l l}<8.8 \mathrm{TeV}$ is used to further reduce the backgrounds and optimize the signals. In this signal region with $p_{T}^{l}>1 \mathrm{TeV}$, the $p p \rightarrow V V, t \bar{t}$ background becomes almost negligible. The number of signal and background events after implementing these cuts are shown in Table IX.

The expected number of signal events for both BP1 and BP2 at the $14 \mathrm{TeV}$ run of the LHC with luminosity $L=$ $3000 \mathrm{fb}^{-1}$ becomes less than unity because of the small production cross section (see Table IX). However, for the LHC run at $27 \mathrm{TeV}$ with $L=3000 \mathrm{fb}^{-1}$, the dilepton final state channels produce a large number of signal events satisfying all the above-mentioned cuts. The significance of the signal over background ( $\frac{S}{\sqrt{B}}$ for $S, B>3$ ) attains a value of 13.45(8.87) for BP1(BP2) for the HE-LHC. Hence, one can use these results to discover/exclude the heavy $Z_{R}$ boson through this channel. 


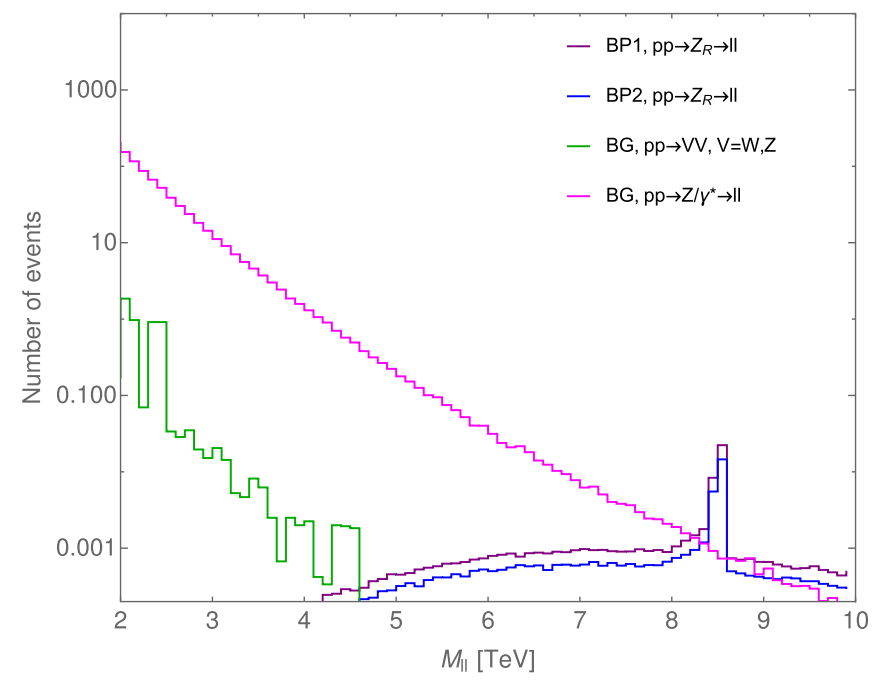

(a)

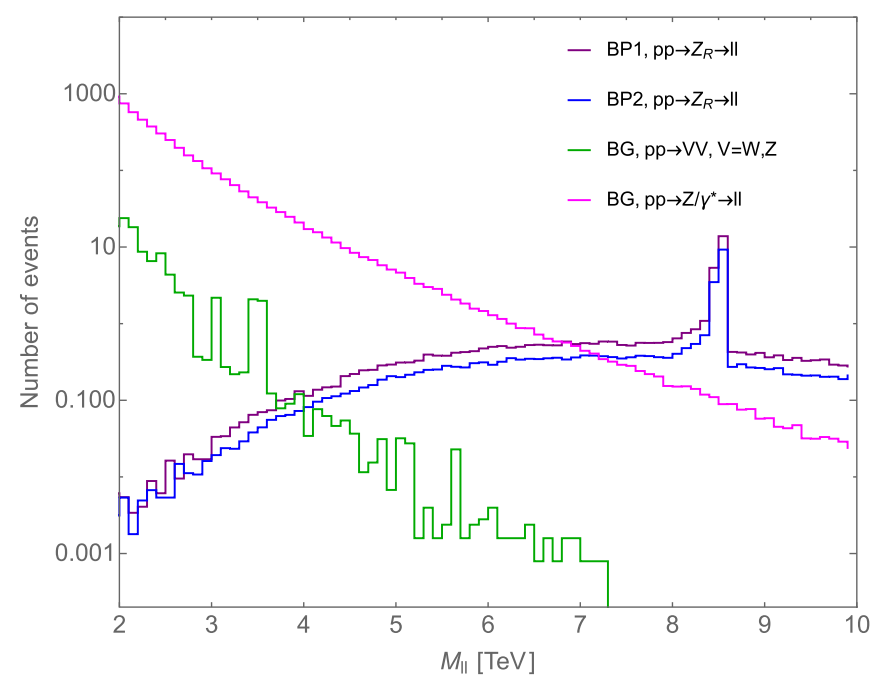

(b)

FIG. 2. The invariant mass distribution of the SFOS pair of leptons. The signal as well as the backgrounds are selected with $p_{T}^{l}>30 \mathrm{GeV}(l=e, \mu)$ and $|\eta|<2.5$. The purple and blue lines stand for the signal $p p \rightarrow l l$ events corresponding to BP1 and BP2, respectively, whereas the backgrounds are indicated by the green and magenta lines. The left plot is in the context of the LHC at $14 \mathrm{TeV}$ and right plot is for $27 \mathrm{TeV}$. The integrated luminosity $3000 \mathrm{fb}^{-1}$ remains the same for all these processes.

In these searches, only the $Z_{R} \rightarrow l l,(l=e, \mu)$ decay modes have been considered. However, the $Z_{R} \rightarrow \tau \tau$ can potentially enhance the signal as $\tau$ can give one lepton (electron or muon) in the final state through its decay via the virtual $W$ boson. This contribution remains small as the final state electron or muon can come via the decay of the virtual gauge bosons, which is suppressed by the branching and the chosen selection and optimization cuts $p_{T}^{l}>1 \mathrm{TeV}$ and $8.0<M_{l l}<8.8 \mathrm{TeV}$. The number of events coming from the $\tau \tau$ channel at the $14 \mathrm{TeV}$ run of the LHC is always less than one event and not considered further. On the other hand, the number of events for the $Z_{R} \rightarrow \tau \tau$ channel can be enhanced by $0.002(0.00)$ for BP1(BP2) at $27 \mathrm{TeV}$ LHC and the corresponding significance for BP1 and BP2 remains the same.

\section{B. Dijet searches}

The heavy gauge bosons $Z_{R}$ and $W_{R}$ both can decay directly into two quarks giving rise to dijet final states that we study in the context of 14 and $27 \mathrm{TeV}$ LHC experiments. An experimental search for the heavy gauge bosons has already been performed [17] in the dijet channel and here we follow a similar strategy. The events are selected with at least two anti- $k_{t}$ jets with jet cone size 0.4 and having transverse momentum $p_{T}$ greater than $1 \mathrm{TeV}$. Also the candidate jet is required to satisfy a pseudorapidity cut of $|\eta|<2.5$. Similar to the dilepton search, the dijet invariant mass distribution $M_{j j}$ can be a useful probe to search for $W_{R}$ and $Z_{R}$ gauge bosons. The dominant SM background for the dijet signal arises from the $p p \rightarrow j j$ process. Other processes including $p p \rightarrow V V, V=W, Z$, and $t \bar{t}$ also add to the SM background but these contributions are extremely small and can be safely neglected. The invariant mass distribution of the signal as well as background events is shown in Fig. 3 for LHC energy $\sqrt{s}=$ $14 \mathrm{TeV}$ (left) and $\sqrt{s}=27 \mathrm{TeV}$ (right). The purple and blue dotted lines indicate the hadronic decay of the $W_{R}$, whereas the purple and blue solid lines are those for the $Z_{R}$ gauge boson. A signal region $4.2<M_{j j}<5.3 \mathrm{TeV}$ has been used to optimize the significance for the $W_{R}$ gauge boson search. In this choice, the expected number of signal events attains values of 1067.25 (705.99) for BP1 (BP2) at $14 \mathrm{TeV}$ LHC with $L=3000 \mathrm{fb}^{-1}$. The significances become 1.18(0.78) due to the large irreducible SM

TABLE IX. The signal as well as the backgrounds are selected with $p_{T}^{l}>1 \mathrm{TeV}(l=e, \mu)$ and $|\eta|<2.5$ to reduce the background contribution. The signal $p p \rightarrow Z_{R} \rightarrow l l$ and the background are obtained after optimization cuts $8.0<M_{l l}<8.8$ TeV. The dijet (jet misidentified as lepton) background is also reduced by these choice of selection $p_{T}^{j}>1 \mathrm{TeV}$ and optimization $8.0<M_{j j}<8.8 \mathrm{TeV}$.

\begin{tabular}{|c|c|c|c|c|c|c|c|c|c|c|}
\hline \multirow[b]{2}{*}{ Energy $[\mathrm{TeV}]$} & \multicolumn{6}{|c|}{ SM background for the signal $p p \rightarrow Z_{R} \rightarrow l l$} & \multicolumn{2}{|c|}{ Total signal events } & \multicolumn{2}{|c|}{ Significance } \\
\hline & $p p \rightarrow Z / \gamma^{*} \rightarrow l l$ & $p p \rightarrow j j$ & $Z Z$ & $W Z$ & $W W$ & $t \bar{t}$ & BP1 & BP2 & BP1 & BP2 \\
\hline 14 & 0.009 & 0.001 & 0 & 0 & 0 & 0 & 0.0037 & 0.0025 & $\ldots$ & . \\
\hline 27 & 0.88 & 2.18 & 0 & 0 & 0 & 0 & 23.51 & 15.51 & 13.45 & 8.87 \\
\hline
\end{tabular}




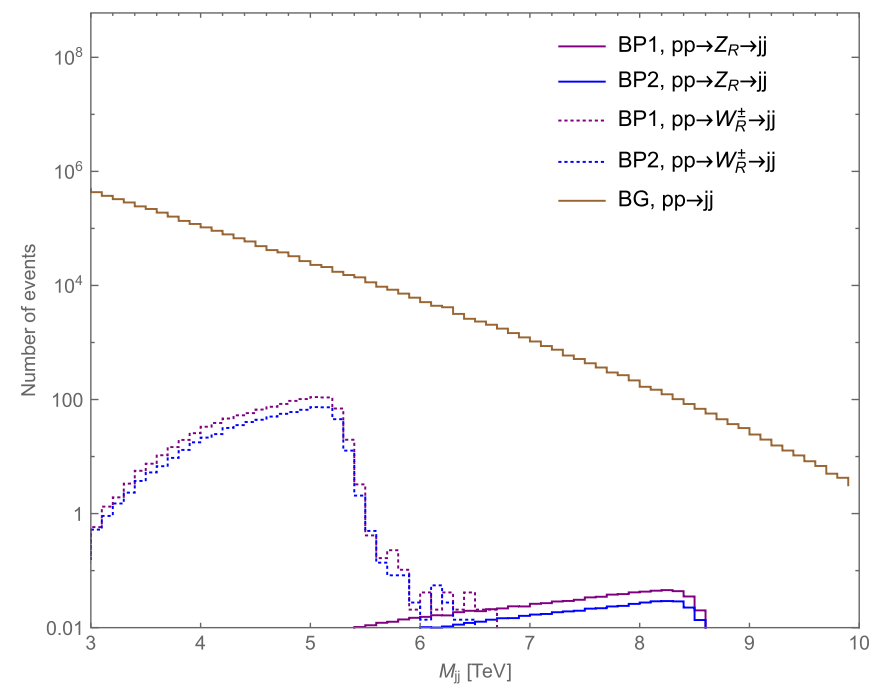

(a)

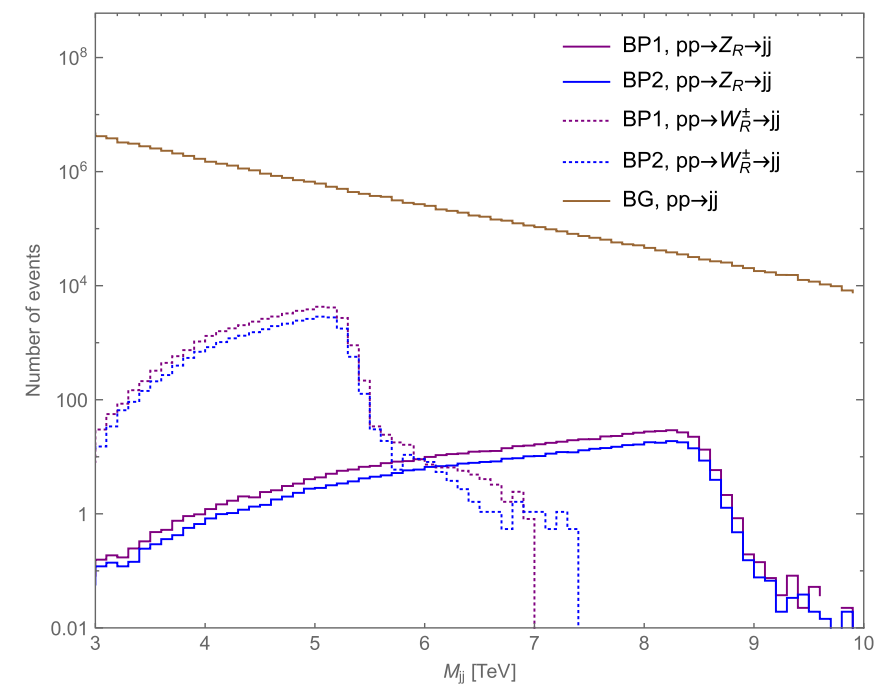

(b)

FIG. 3. The dijet invariant mass distribution and the signal process $p p \rightarrow W_{R}^{ \pm} \rightarrow j j$ are denoted by doted purple and blue lines. Whereas the solid line stands for the $p p \rightarrow Z_{R} \rightarrow j j$. The left plot is drawn in the context of the LHC at $14 \mathrm{TeV}$ and right plot for $27 \mathrm{TeV}$. The integrated luminosity $3000 \mathrm{fb}^{-1}$ remains the same for all these processes. The dominant SM background $p p \rightarrow j j$ distribution is indicated by the brown line. Here in these plots, the signal as well as the background are selected with $p_{T}^{j}>1 \mathrm{TeV}$ and $|\eta|<2.5$ along with the jet's con size 0.4 .

background events with large number of events $N_{p p \rightarrow j j}=$ $8.178 \times 10^{4}$. At the $27 \mathrm{TeV}$ LHC with a luminosity of $3000 \mathrm{fb}^{-1}$, the signal events increase to $4.129 \times 10^{4}$ $\left(2.753 \times 10^{4}\right)$ for BP1 (BP2) while the background events become $3.172 \times 10^{6}$. This results in an increase of the signal to background significance to 23.19 (15.46) for BP1 (BP2), respectively, in this case.

A more stringent cut on the signal and background regions with $p_{T}^{j}>1.5 \mathrm{TeV}$ for the $W_{R}$ search can result in a better significance. Though this cut reduces the signal as well as background events, the background is affected more since the signal jets are arising from the decay of a heavy resonance and can have larger $p_{T}$. If we choose the same signal region $4.2<M_{j j}<5.3 \mathrm{TeV}$ as before, the signal significances attain values of 1.77 (1.17) for $14 \mathrm{TeV}$ LHC with $L=3000 \mathrm{fb}^{-1}$ while the same becomes 33.67 (22.42) for $27 \mathrm{TeV}$ LHC with $L=3000 \mathrm{fb}^{-1}$ for BP1 (BP2) parameter space. The corresponding numbers are quoted in Table X.

The large mass of the $Z_{R}$ boson results in a small production cross section at the $14 \mathrm{TeV}$ LHC. Thus the expected numbers of the signal $p p \rightarrow Z_{R} \rightarrow j j$ events with $p_{T}^{j}>1 \mathrm{TeV}$ at the $14 \mathrm{TeV}$ LHC with $L=3000 \mathrm{fb}^{-1}$ remain small as can be seen in the distribution plot in Fig. 3. For a favorably chosen signal region with a dijet invariant mass $7.15<M_{j j}<8.65 \mathrm{TeV}$, the expected number of events is negligible compared to around 8000 background events at the $14 \mathrm{TeV}$ LHC with $3000 \mathrm{fb}^{-1}$ luminosity. The expected numbers for $27 \mathrm{TeV}$ LHC are found to be around 300 signal events compared to $6.542 \times 10^{4}$ background events. Hence the significances are extremely poor and one is not able to identify a $Z_{R}$ boson in this case. As before, we can try to increase the required $p_{T}$ of the jets to check if the signal significance improves. We select the signal and background events with $p_{T}^{j}>3 \mathrm{TeV}$. This yields only one expected signal event compared to about 200 background events at the LHC $14 \mathrm{TeV}$ (see Table XI). However, the expected number as well as the significance increase at $27 \mathrm{TeV}$ with $L=3000 \mathrm{fb}^{-1}$. The number of signal events is found to be 260.12 (166.32) and the corresponding significance attains a value of 1.42 (0.91) for BP1 (BP2). Hence, we

TABLE X. Total number of events of the signal $p p \rightarrow W_{R}^{ \pm} \rightarrow j j$ and SM background with $p_{T}^{j}>1.5 \mathrm{TeV}$ and $|\eta|<2.5$ along with cone size of 0.4 at the 14 and $27 \mathrm{TeV}$ run of the LHC with $L=3000 \mathrm{fb}^{-1}$ obtained after the optimization cut $4.2<M_{j j}<5.3 \mathrm{TeV}$.

\begin{tabular}{|c|c|c|c|c|c|c|c|c|c|}
\hline \multirow[b]{2}{*}{ Energy $[\mathrm{TeV}]$} & \multicolumn{5}{|c|}{ SM background for the signal $W_{R}^{ \pm} \rightarrow j j$} & \multicolumn{2}{|c|}{ Total signal events } & \multicolumn{2}{|c|}{ Significance } \\
\hline & $p p \rightarrow j j$ & $Z Z$ & $W Z$ & $W W$ & $t \bar{t}$ & BP1 & $\mathrm{BP} 2$ & BP1 & BP2 \\
\hline 14 & $2.895 \times 10^{5}$ & 0 & 0 & 0 & 0 & 952.681 & 632.021 & 1.77 & 1.17 \\
\hline 27 & $1.215 \times 10^{6}$ & 0 & 0 & 0 & 0 & $3.711 \times 10^{4}$ & $2.471 \times 10^{4}$ & 33.67 & 22.42 \\
\hline
\end{tabular}


TABLE XI. Total number of events of the signal $p p \rightarrow Z_{R} \rightarrow j j$ and background with $p_{T}^{j}>3 \mathrm{TeV}$ along with cone size 0.4 at the 14 and $27 \mathrm{TeV}$ run of LHC with $L=3000 \mathrm{fb}^{-1}$ are obtained after the optimization cut $7.15<M_{j j}<8.65 \mathrm{TeV}$.

\begin{tabular}{|c|c|c|c|c|c|c|c|c|c|}
\hline \multirow[b]{2}{*}{ Energy $[\mathrm{TeV}]$} & \multicolumn{5}{|c|}{ SM background for the signal $Z_{R} \rightarrow j j$} & \multicolumn{2}{|c|}{ Total signal events } & \multicolumn{2}{|c|}{ Significance } \\
\hline & $p p \rightarrow j j$ & $Z Z$ & $W Z$ & $W W$ & $t \bar{t}$ & BP1 & BP2 & BP1 & $\mathrm{BP} 2$ \\
\hline 14 & 297.34 & 0 & 0 & 0 & 0 & 0.40 & 0.26 & $\ldots$ & $\ldots$ \\
\hline 27 & $3.334 \times 10^{4}$ & 0 & 0 & 0 & 0 & 260.12 & 166.32 & 1.42 & 0.91 \\
\hline
\end{tabular}

need large energy and luminosity to observe signatures of such a heavy $8.5 \mathrm{TeV}$ neutral gauge boson through the dijet final state channel in this LRSUSY model.

In the above analysis, anti- $k_{t}$ jets with cone size 0.4 have been considered and the corresponding invariant mass distribution was shown in Fig. 3 for the selected events with $p_{T}^{j}>1 \mathrm{TeV}$ and $|\eta|<2.5$. It is important to note that if we increase the jets' cone size to 1.0, the number of events corresponding to the signal as well as the background increases by $\sim 20 \%$. Hence the significance is enhanced by $\sim 10 \%$.

We are now ready to discuss the various SUSY decay channels for the heavy gauge bosons and their implications in the context of the LHC experiment. The presence of light SUSY particles in the spectrum allows the heavy gauge bosons to decay into these light states, which can lead to interesting new channels. The branching fraction of the heavy gauge bosons decaying into light electroweakinos can be quite large depending upon the choice of LRSUSY parameters. The charginos and neutralinos produced from the decay of heavy gauge bosons can themselves undergo cascade decays giving rise to final state leptons and jets with the LSP remaining undetected. Thus these signals are quite different compared to conventional search channels due to the presence of large missing energy in the final state. A large ensemble of final states can arise from the SUSY decays of the heavy gauge bosons. Here we mainly focus on the leptonic cascade decay modes arising from the mono- $X+E_{T}(X=W, Z)$ channels [46] in the context of the HL-LHC and HE-LHC experiments. The main motivations for choosing these channels are as follows: (i) These final states are well understood as they have already been experimentally studied in the context of dark matter searches by the ATLAS and CMS collaborations $[47,48]$.

(ii) We restrict ourselves to leptonic decay channels for the SM gauge bosons as these produce relatively clean channels that are easy to identify in a hadronrich environment like the LHC experiment.

Figure 4 depicts a couple of examples where the heavy gauge boson SUSY decays can lead to final states with a SM gauge boson plus large $\mathbb{E}_{T}$.

One can also encounter relatively complex cascade decays, with multiple decay chains in between, which may eventually lead to final states with multiple leptons and jets along with large missing transverse energy. As an example let us consider the following decay chains $W_{R}^{ \pm} \rightarrow \tilde{\chi}_{1}^{\mp} \tilde{\chi}^{ \pm \pm}$or $Z_{R} \rightarrow \tilde{\chi}^{\mp \mp} \tilde{\chi}^{ \pm \pm}$, with $\tilde{\chi}^{ \pm \pm} \rightarrow \tilde{\chi}_{1}^{0} H_{1}^{ \pm \pm}$or $\tilde{\chi}^{ \pm \pm} \rightarrow \tilde{\chi}_{1}^{ \pm} W^{ \pm}$, and finally $H_{1}^{ \pm \pm} \rightarrow l^{ \pm} l^{ \pm}$while $\tilde{\chi}_{1}^{ \pm} \rightarrow W^{ \pm} \tilde{\chi}_{1}^{0}$ with $W^{ \pm} \rightarrow l^{ \pm} \nu_{l} / q q^{\prime}$. The detailed collider analysis of these channels is beyond the scope of the current paper and is not discussed here.

For our collider analysis we chose two benchmark points as was discussed before. The first benchmark point BP1 is optimized for the mono- $W+\mathbb{E}_{T}$ searches, where the lightest neutralino and the lightest chargino are $S U(2)_{R}$ wino dominated. In BP1, $\operatorname{BR}\left(\tilde{\chi}_{1}^{ \pm} \rightarrow \tilde{\chi}_{1}^{0} W^{ \pm}\right)=100 \%$ and the total contribution coming from $W_{R}^{ \pm}$to $\tilde{\chi}_{1}^{ \pm} \tilde{\chi}_{1}^{0}$ is about $26.8 \%$. Our second benchmark point, BP2, is more suitable for the mono- $Z+\mathscr{E}_{T}$ searches where both $\tilde{\chi}_{1}^{0}$ and $\tilde{\chi}_{2}^{0}$ are substantially consisting of Higgsino components $\tilde{\delta}^{c^{0}}$ and $\tilde{\bar{\delta}}^{c^{0}}$ of the triplet Higgs bosons while the lightest chargino $\tilde{\chi}_{1}^{ \pm}$is also

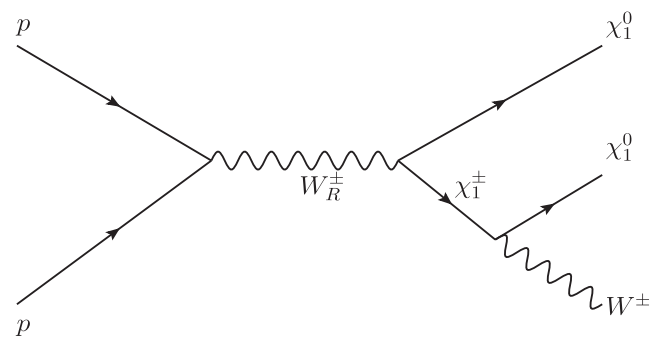

(a)

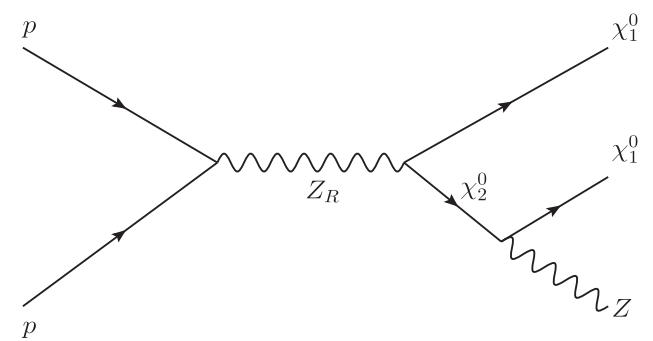

(b)

FIG. 4. Mono- $X+\mathbb{E}_{T}(X=W, Z)$ channels through one-step cascade of the heavy gauge bosons. 
Higgsino (triplet) dominated. Thus the $\operatorname{BR}\left(W_{R}^{ \pm} \rightarrow \tilde{\chi}_{1}^{0} \tilde{\chi}_{1}^{ \pm}\right)$is only around $10.37 \%$ while $\operatorname{BR}\left(Z_{R} \rightarrow \tilde{\chi}_{1}^{0} \tilde{\chi}_{2}^{0}\right)=31.16 \%{ }^{2}$ and $\operatorname{BR}\left(\tilde{\chi}_{2}^{0} \rightarrow Z+\tilde{\chi}_{1}^{0}\right)=95 \%$.

\section{Mono- $X$ plus missing transverse energy}

Events with a single $W / Z$ boson accompanied by large missing transverse energy constitute a very clean and distinctive signature in new physics searches at the LHC. This topology has been thoroughly analyzed by both the ATLAS and CMS collaborations [47,48], mainly in the context of DM searches. In this work, we follow these search channels to probe the heavy gauge bosons for the chosen benchmark points. We present these searches for the future collider perspective, assuming the LHC will operate at the CoM energies of $\sqrt{s}=14$ and $27 \mathrm{TeV}$ with an integrated luminosity of $3000 \mathrm{fb}^{-1}$.

\section{Mono- $W+\mathbb{E}_{T}$ searches}

We perform a search for the heavy charged gauge bosons $W_{R}$ in events where a $W$ boson is produced through onestep cascade decay [see Fig. 4(a)] of the chargino $\tilde{\chi}_{1}^{ \pm}$. Here we only consider the leptonic decay channel of the $W$ boson $(W \rightarrow l \nu, l=e, \mu)$.

The signal event would be characterized by the presence of a high $p_{T}$ lepton (electron and muon) and a large $\mathbb{E}_{T}$ imbalance due to the undetected escaping neutrino and lightest neutralinos. The search strategy reported in Ref. [47], which focused on the DM searches, has been followed with suitable modifications aimed to optimize the signal significance. The event is selected with one isolated lepton (electron or muon) having transverse momentum $p_{T}$ greater than $400 \mathrm{GeV}$. The lepton isolation criteria are the same as in the previous case. Also the candidate electron (muon) is required to satisfy the rapidity cut $|\eta|<2.5$. The main discriminating variable used in this search is the transverse mass defined as $M_{T}=$ $\sqrt{2 p_{T}^{l} \mathbb{E}_{T}\left(1-\cos \Delta \phi_{l, \not \mathbb{H}_{T}}\right)}$, where $p_{T}^{l}$ is the transverse momentum of the charged lepton and $\Delta \phi_{l, \not_{T}}$ is the difference in azimuthal angle between the lepton transverse momentum and missing transverse energy $\mathbb{E}_{T}$.

One of the main sources of SM background is the $p p \rightarrow l \nu+j j$ production channel. Besides, processes like $V V(V=W, Z), t \bar{t}$, etc., also contribute to the background. Among the $V V$ processes, the contribution comes from $Z Z \rightarrow l l \nu \nu, W Z \rightarrow l \nu \nu \nu$, and $W W \rightarrow l \nu l \nu$ channels where the additional charged leptons get misidentified or remain unreconstructed.

The transverse mass and missing energy distribution for the number of signal $S$ as well as background $B$ events at $\sqrt{s}=14 \mathrm{TeV}$ (left) and $27 \mathrm{TeV}$ (right) with integrated

\footnotetext{
${ }^{2}$ The large coupling of $Z_{R}$ with the neutralinos is due to them being triplets of $S U(2)_{R}$ in this case.
}

luminosity $L=3000 \mathrm{fb}^{-1}$ are shown in Figs. 5 and 6, respectively. The purple and blue solid lines indicate the signal events for BP1 and BP2, respectively. In general, the direct decay of the heavy charged boson can also give final state with one lepton plus missing energy (for example, $W_{R}^{ \pm} \rightarrow l \nu$ ) but this channel is absent for the chosen parameter spaces in this model. Hence, we do not observe any peak in the $M_{T}$ distribution at $M_{W_{R}} \sim 5.25 \mathrm{TeV}$. This is a major difference of this model compared to the other models. In both the figures, the dominant SM $p p \rightarrow l \nu j j$ background is represented by the red line, whereas the green line is for the SM $p p \rightarrow V V(V=W, Z)$ background. The $p p \rightarrow t \bar{t}$ remain almost 0 for the chosen signal regions (see Table XII); hence it is not demonstrated in these figures. The number of events are calculated as $S, B=$ $\epsilon A \sigma L$, where $\epsilon, A$ stand for the efficiency and acceptance for the signal or background events, $\sigma$ indicates the cross section of the final state events, and luminosity is denoted by $L$.

Several combinations of selection variables $M_{T}$ and $\mathbb{E}_{T}$ are investigated. Among them, a signal region with $1.75<$ $M_{T}<3.0 \mathrm{TeV}$ and $\mathscr{E}_{T}>0.95 \mathrm{TeV}$ is chosen, which yields the most efficient optimization of signal significance. The expected number of signal and background events in this region are shown in Table XII. The expected number of the signal events for $\mathrm{BP} 1$ at the $14 \mathrm{TeV}$ run of the LHC with luminosity $L=3000 \mathrm{fb}^{-1}$ is 24.12 . This number remains less than unity for BP2. The LHC run at $27 \mathrm{TeV}$ with $L=$ $3000 \mathrm{fb}^{-1}$ gives a large number of signal events, 1096.73 for BP1 and 42.5 for BP2 due to large production cross section. The SM $p p \rightarrow l \nu j j$ process background remains dominant at $14 \mathrm{TeV}$ as well as $27 \mathrm{TeV}$ even after the optimization cuts. The $p p \rightarrow V V$ background is negligibly small compared to the $p p \rightarrow l \nu j j$ process. It is to be noted that the jet faking lepton (assuming $0.1 \%$ to electron and $0.5 \%$ to muon [49]) in the $p p \rightarrow j j$ channel also gives additional contribution to the background. It is found to be 22.5 and 936.2 events at 14 and $27 \mathrm{TeV}$, respectively, for similar optimization cuts with $1.75<M_{T}^{j, \not \phi_{T}}<3.0 \mathrm{TeV}$ and $\mathbb{E}_{T}>0.95 \mathrm{TeV}$. Signal significance attains a value of 0.54 for BP1 at $14 \mathrm{TeV}$ while it becomes 5.83 at $27 \mathrm{TeV}$. The expected number for the significance of BP2 at $14 \mathrm{TeV}$ as well as $27 \mathrm{TeV}$ remains less than unity. The signal events and corresponding significance for BP1 indicate that the heavy gauge bosons could be discovered at the HE-HL-LHC. Or one can exclude this parameter space if we do not get any signal at a future collider. The BP2 demands that one needs higher energy collider with high integrated luminosity to discover or exclude such a region.

In this analysis, only the $W \rightarrow l \nu,(l=e, \mu)$ decay modes have been considered. However the $W \rightarrow \tau \nu_{\tau}$ can also enhance the signal as tau can give one lepton (electron or muon) in the final state through its decay, e.g., $\tau \rightarrow W \nu_{\tau}$, $W \rightarrow l \nu$. It could be quantified as follows. Using the same 


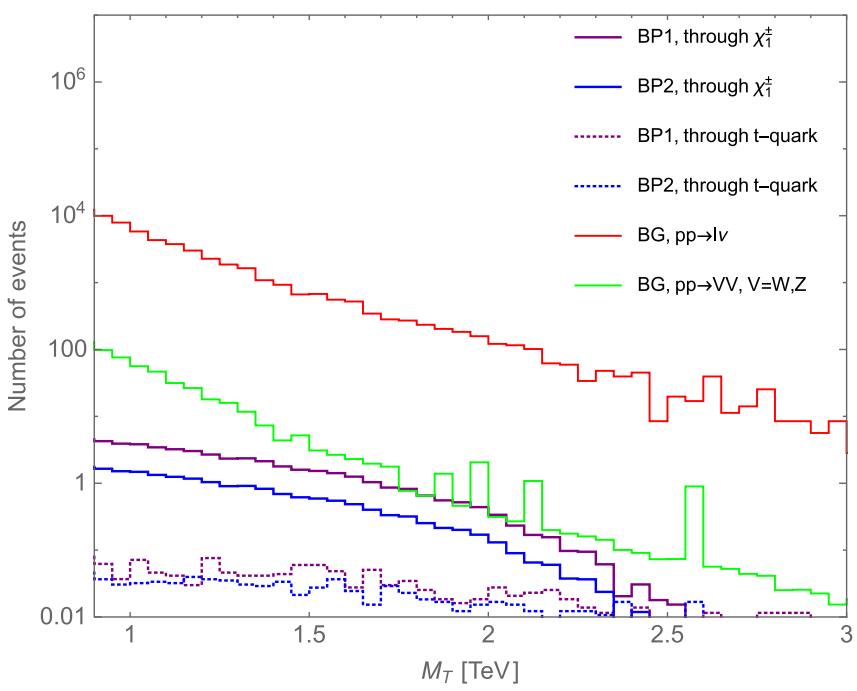

(a)

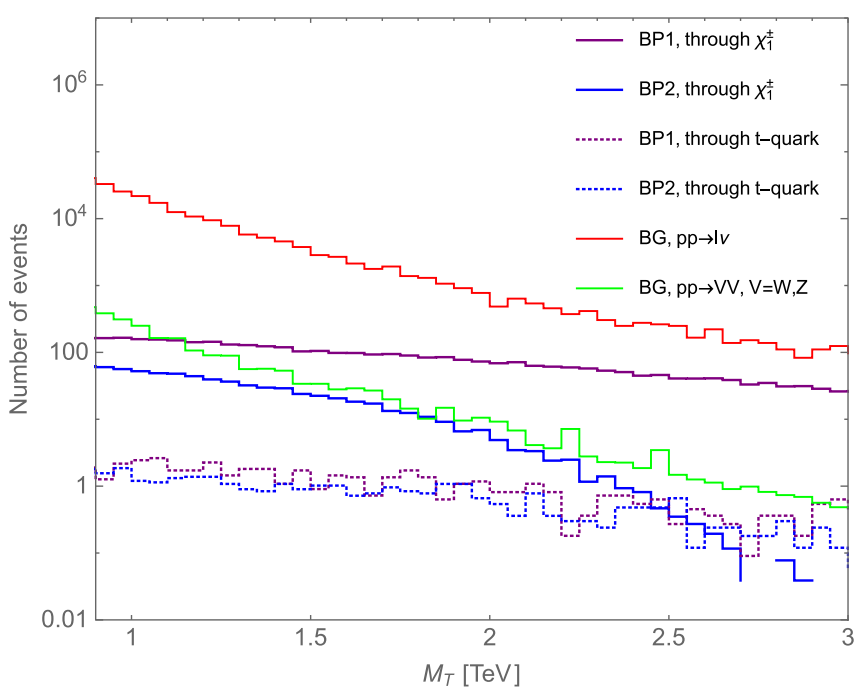

(b)

FIG. 5. The event selection criteria of the signal and background requires at least one lepton $(e$ or $\mu)$ in the event, with each lepton having $p_{T}^{l}>400 \mathrm{GeV}$ and $|\eta|<2.5$. The total number of the signal of events for the process $p p \rightarrow 1 l+\mathbb{E}_{T}(l=e, \mu)$ through the cascade (indicated by the purple and blue lines) of the $W_{R}$ gauge boson in the context of the LHC at (a) $14 \mathrm{TeV}$ and (b) $27 \mathrm{TeV}$ with integrated luminosity $3000 \mathrm{fb}^{-1}$. The other purple and blue dotted lines stand for the additional signal events through another cascade decay process $p p \rightarrow W_{R} \rightarrow t \bar{b}, t \rightarrow W b, W \rightarrow l \nu$. The number of events with $1 l+\mathbb{E}_{T}$ final state in this channel remains less than unity as the lepton isolation cut significantly wanes the signal. The dominant SM $p p \rightarrow l \nu j j$ background is indicated by the red line, whereas the green line stands for SM $p p \rightarrow V V(V=W, Z)$. The contribution comes from $Z Z \rightarrow l l \nu \nu, W Z \rightarrow l \nu \nu \nu$, and $W W \rightarrow l \nu l \nu$ channels where the additional charged leptons get misidentified or remain unreconstructed.

selection and optimization cuts $p_{T}^{l}>400 \mathrm{GeV}, 1.75<$ $M_{T}^{j, \not \phi_{T}}<3.0 \mathrm{TeV}$, and $\boldsymbol{E}_{T}>0.95 \mathrm{TeV}$, it is found that the number of events at the $14 \mathrm{TeV}$ run of the LHC with luminosity $L=3000 \mathrm{fb}^{-1}$ is enhanced by $0.91(0.001)$ for BP1 (BP2). The significance now becomes 0.88 for the

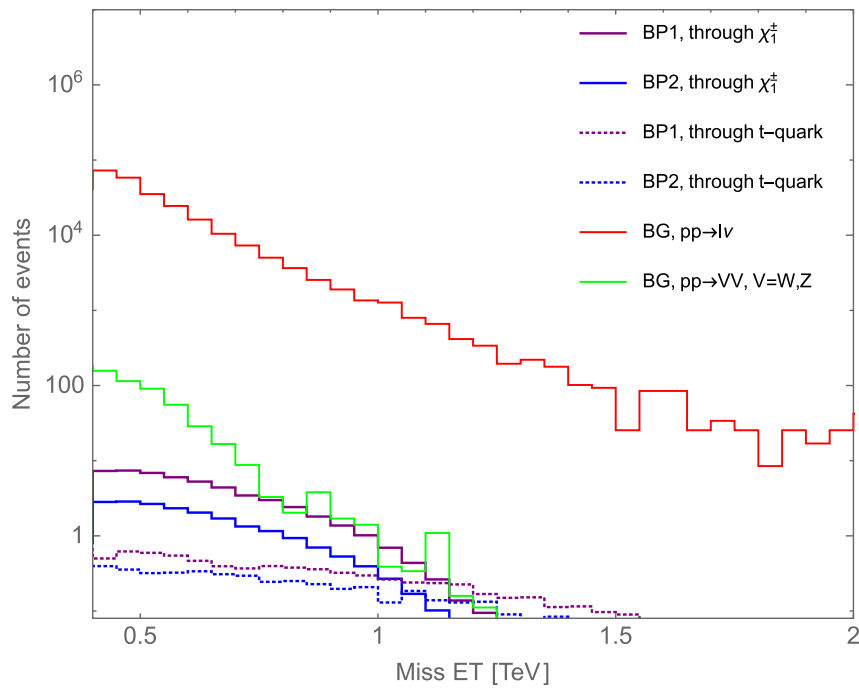

(a)
$\mathrm{BP} 1$. Whereas the number of events for these channels goes to $9.35(0.02)$ for BP1 (BP2) at $27 \mathrm{TeV}$ LHC and the significance corresponding to BP1 and BP2 attains values of 14.61 and 0.57 , respectively.

The $l \nu$ final state can also come through another cascade decay process with $p p \rightarrow W_{R} \rightarrow t \bar{b}, t \rightarrow W b, W \rightarrow l \nu$.

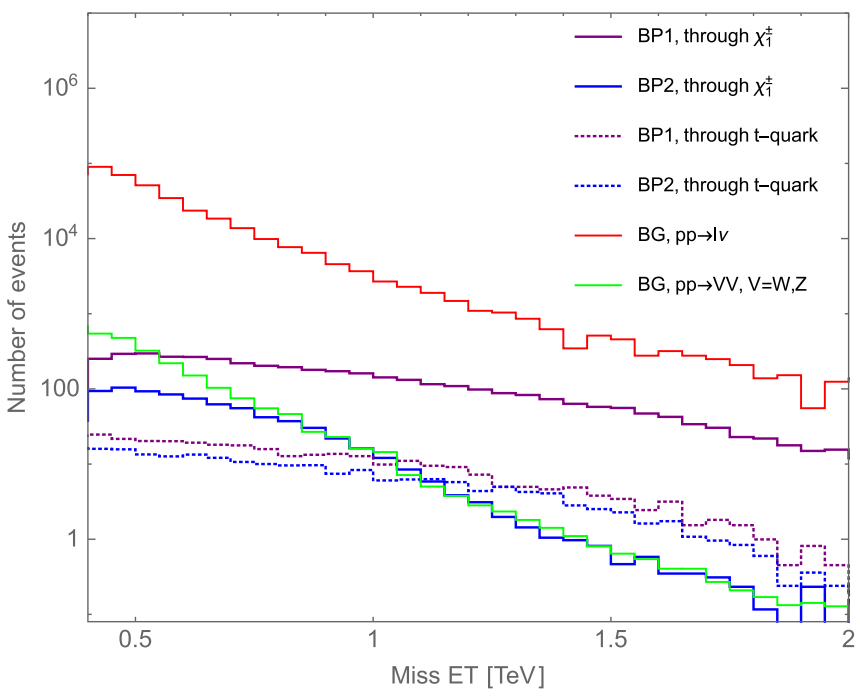

(b)

FIG. 6. The transverse missing energy distribution for the signal $p p \rightarrow 1 l+\mathbb{E}_{T}(l=e, \mu)$ and background events at (a) 14 TeV and (b) $27 \mathrm{TeV}$ with integrated luminosity $3000 \mathrm{fb}^{-1}$. The selection criteria of the events and color coding for the shown lines are the same as in Fig. 5 . 
TABLE XII. The event selection criteria of the signal and background require at least one lepton $(\epsilon$ or $\mu)$ in the event, with each lepton having $p_{T}^{l}>400 \mathrm{GeV}$ and $|\eta|<2.5$. The total number of events of the signal and background at the 14 and $27 \mathrm{TeV}$ run at the LHC with $L=3000 \mathrm{fb}^{-1}$ is obtained after the optimization cuts $1.75<M_{T}<3.0 \mathrm{TeV}$ and $\mathscr{E}_{T}>0.95 \mathrm{TeV}$. The jet faking lepton $p p \rightarrow j j$ channels also add to the background. It is found to be 8.77 and 17.39 at 14 and $27 \mathrm{TeV}$, respectively for the similar optimization cuts $1.75<M_{T}^{j, \not \not_{T}}<3.0 \mathrm{TeV}$ and $\mathscr{E}_{T}>0.8 \mathrm{TeV}$.

\begin{tabular}{|c|c|c|c|c|c|c|c|c|c|}
\hline \multirow[b]{3}{*}{ Energy $[\mathrm{TeV}]$} & \multicolumn{9}{|c|}{$W+\mathbb{E}_{T}, W \rightarrow l \nu_{l}$ final state through charginos $\tilde{\chi}_{1}^{ \pm}$} \\
\hline & \multicolumn{5}{|c|}{ SM backgrounds } & \multicolumn{2}{|c|}{ Total signal events } & \multicolumn{2}{|c|}{ Significance } \\
\hline & $W \rightarrow l \nu_{l}$ & $Z Z$ & $W Z$ & $W W$ & $t \bar{t}$ & BP1 & BP2 & BP1 & $\mathrm{BP} 2$ \\
\hline 14 & 758.63 & 0.02 & 0.15 & 2.04 & 0 & 24.12 & 0.60 & 0.87 & \\
\hline 27 & 5577.42 & 7.08 & 19.59 & 23.93 & 0 & 1096.73 & 42.5 & 14.6 & 0.56 \\
\hline
\end{tabular}

It can be seen from the BPs that the branching of the heavy charged gauge boson into the top-bottom quark final state is quite large. Also these particles are emitted almost back to back and remain boosted with $p_{T}^{t, \bar{b}} \sim 2 \mathrm{TeV}$ for $M_{W_{R}}=5.25 \mathrm{TeV}$. Hence the separation $\Delta R_{l b}$ between the final state bottom quark and lepton (both coming from decay of the top quark) becomes very small. It can also be understood from the parton level distributions shown in Fig. 7. The $\Delta R_{l b}$ distribution of the bottom quark and lepton is demonstrated in Fig. 7(a) while the correlation plot between the final state lepton transverse momentum against $\Delta R_{l b}$ is shown in Fig. 7(b). As the charged lepton isolation demands that there is no other charged particle with $p_{T}>0.5 \mathrm{GeV}$ within a cone of $\Delta R<0.5$, the number of lepton events in the final state passing this criterion is extremely small. Furthermore, the selection cut of $p_{T}^{l}>$ $400 \mathrm{GeV}$ also significantly decreases the events at the analysis level. The isolation and selection criteria decrease the final state lepton events by $\sim 85 \%$. The remaining number of lepton events can be identified as the purple points on the upper and right side of the black dashed lines in the Fig. 7(b). The corresponding transverse mass and missing energy distribution of these events for the BP1 (dotted purple line) and BP2 (dotted blue line) are shown in Figs. 5 and 6, respectively. This cascade decay process can thus contribute to our signal events though the enhancement in the signals remains negligible. Further, a simple b-jet veto will completely kill these events at the analysis level.

\section{Mono- $Z+E_{T}$ searches}

We perform a search for the heavy neutral gauge boson $Z_{R}$ in events where a $Z$ boson is produced through one-step cascade decays [see Fig. 4(b)] of the neutralino $\tilde{\chi}_{2}^{0}$. Here we assume that the $Z$ boson decays leptonically $(Z \rightarrow l l$, $l=e, \mu)$. These events also contain significant missing transverse energy coming from the LSP $\tilde{\chi}_{1}^{0}$.

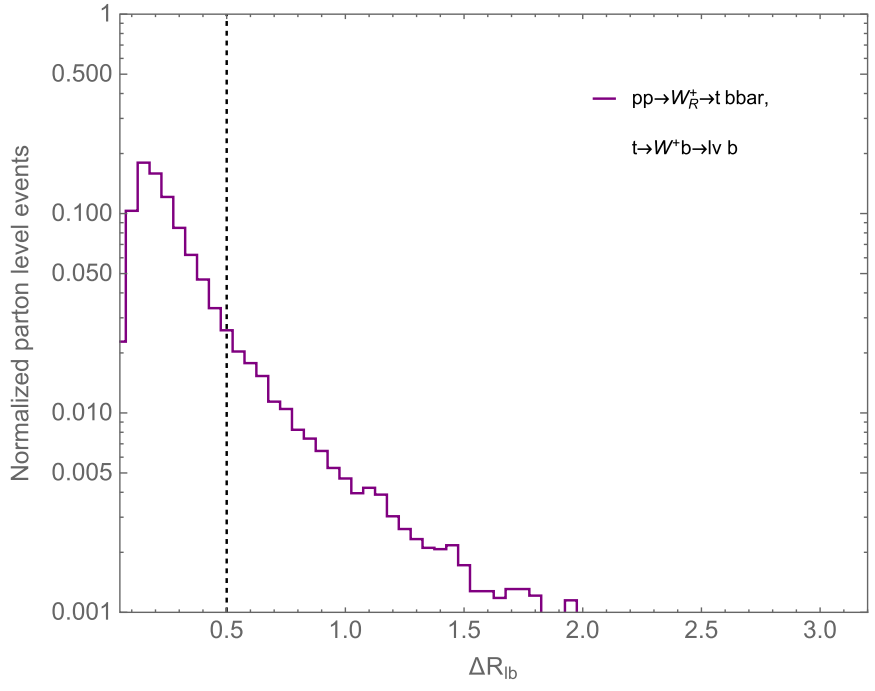

(a)

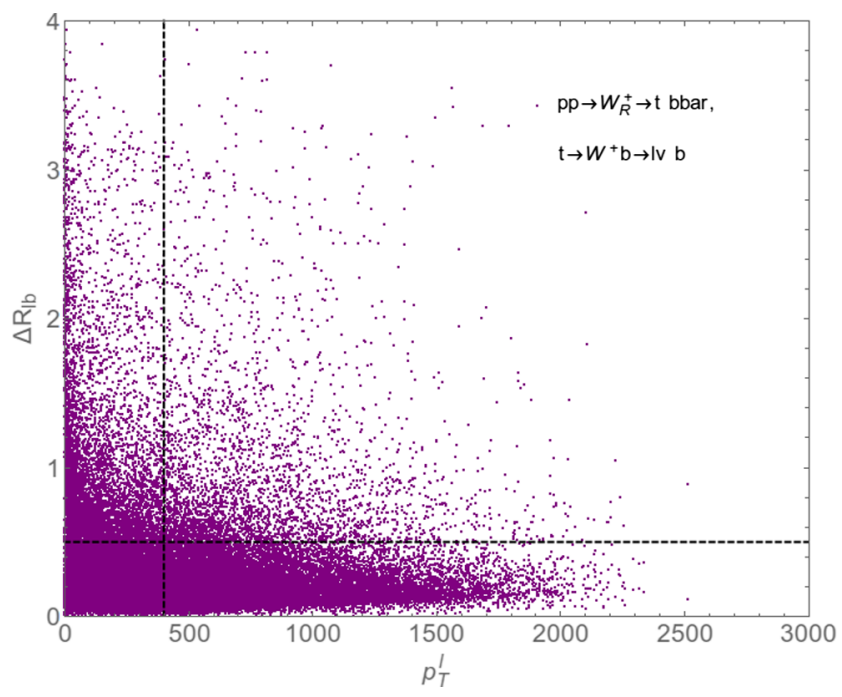

(b)

FIG. 7. (a) The $\Delta R_{l b}$ parton level distribution for the signal $p p \rightarrow W_{R}^{+} \rightarrow t \bar{b}, t \rightarrow W b, W \rightarrow l \nu$ events in the parton level at $\sqrt{s}=14 \mathrm{TeV}$. 


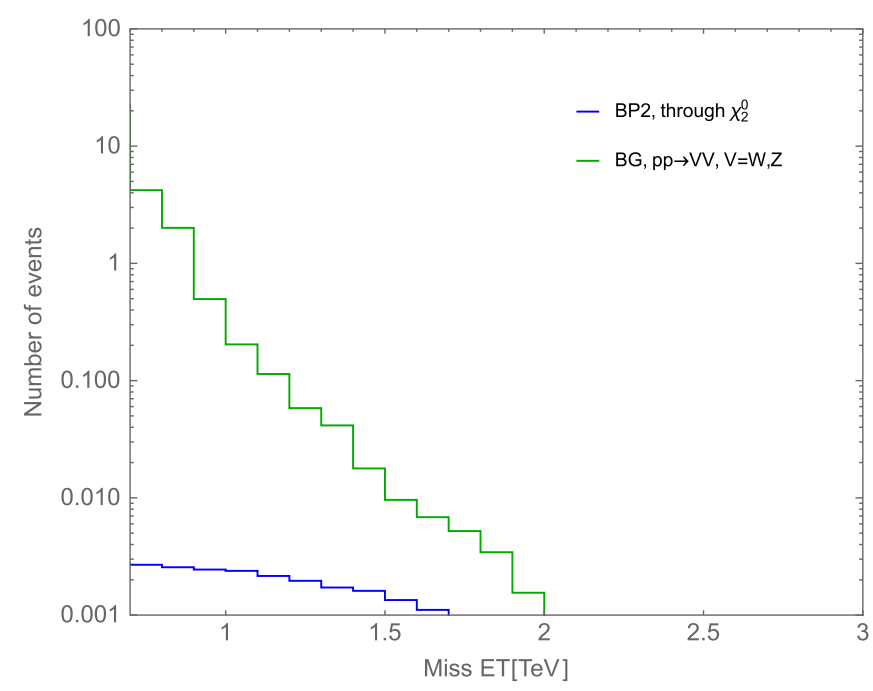

(a)

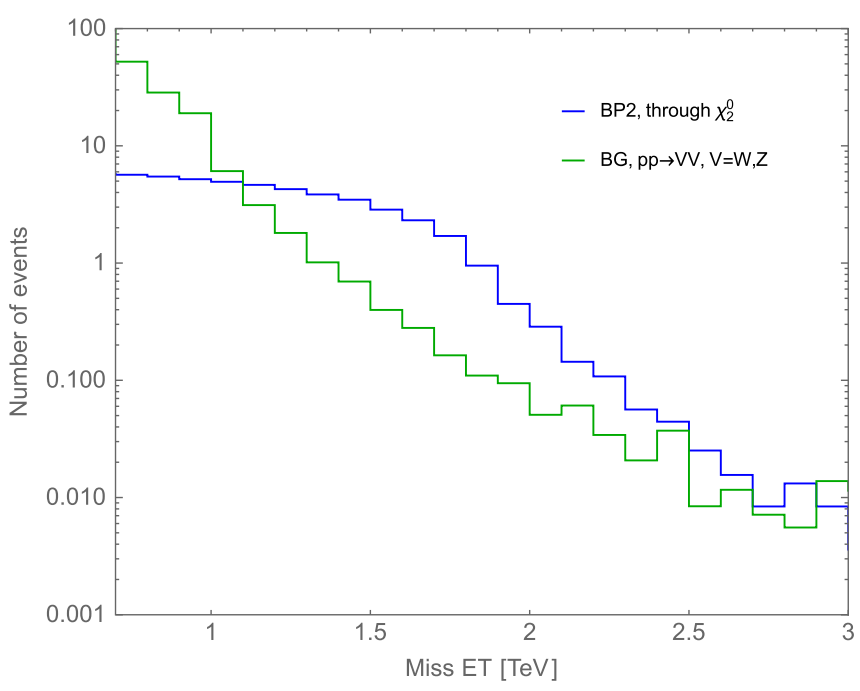

(b)

FIG. 8. The event selection criteria of the signal and background require at least two leptons $(e$ or $\mu)$ in the event, with each lepton having $p_{T}^{l}>30 \mathrm{GeV}$ and $|\eta|<2.5$. The total number of events for the signal process $p p \rightarrow 2 l(l=e, \mu)$ through the cascade and direct decay (indicated by the purple and blue lines) of the $Z_{R}$ gauge boson in the context of the LHC at (a) $14 \mathrm{TeV}$ and (b) $27 \mathrm{TeV}$ with integrated luminosity $3000 \mathrm{fb}^{-1}$. The dominant $\mathrm{SM} p p \rightarrow V V, V=W, Z$ backgrounds are indicated by the green line.

The events are selected with two SFOS isolated electrons or muons with transverse momentum $p_{T}$ larger than $30 \mathrm{GeV}$ satisfying $\left|M_{l l}-M_{Z}\right|<15 \mathrm{GeV}$. Here $M_{l l}$ stands for the invariant mass of the SFOS leptons pair and $M_{Z}=91.1876 \mathrm{GeV}$ is the SM $Z$ boson mass. The isolation criteria are the same as in the previous cases. The charged lepton candidates are required to be within a pseudorapidity range of $|\eta|<2.5$ [48]. The transverse missing energy distribution $\mathbb{E}_{T}$ can be a useful probe to search for the $Z_{R}$ gauge boson. Here processes like $p p \rightarrow Z Z(Z \rightarrow l l, Z \rightarrow \nu \bar{\nu}), p p \rightarrow Z W(Z \rightarrow l l, W \rightarrow l \nu)$, and $p p \rightarrow W W(W \rightarrow l \nu)$ can add to the SM background if additional charged leptons get misidentified or remain unreconstructed. Also other reducible backgrounds like $p p \rightarrow t \bar{t}, t \rightarrow W b, W \rightarrow l \nu$ may also produce two leptons and jets in the final state. The missing energy distribution for the signal as well as background events at a c.m. energy of $\sqrt{s}=14 \mathrm{TeV}$ and $\sqrt{s}=27 \mathrm{TeV}$ (right) with luminosity $L=3000 \mathrm{fb}^{-1}$ are shown in Fig. 8. Please note that the signal events for $\mathrm{BP} 1$ are 0 as $\operatorname{BR}\left(Z_{R} \rightarrow \tilde{\chi}_{1}^{0} \tilde{\chi}_{2}^{0}\right)=0$ in this case. The blue line indicates the signal events distribution for the choice of BP2. The other lines (cyan and green) stand for the SM backgrounds. Also in this case, a signal region $\mathbb{E}_{T}>0.107 \mathrm{TeV}$ is used to optimize the significance. The expected signal and background events are shown in Table XIII. We find that all these SM processes including $p p \rightarrow j j$ (where jets fake as leptons) backgrounds remain small for $\mathrm{LHC}$ run at $14 \mathrm{TeV}$ for these signal regions. Also the number of signal events remains small due to the small production cross section $\sigma\left(p p \rightarrow Z_{R}\right)=0.001 \mathrm{fb}$ at this energy. However, the expected number of signal events for BP2 becomes 7.26 for the LHC run at $27 \mathrm{TeV}$ with integrated luminosity $L=3000 \mathrm{fb}^{-1}$. The corresponding dominant background from $p p \rightarrow V V$ goes to 3.0. The signal significance thus comes out to be 4.2. It is clear from this section that the expected numbers for BP1 can give an indication of a heavy charged boson, whereas BP2 can give hints for the heavy

TABLE XIII. The event selection criteria of the signal and background require at least two leptons $(\epsilon$ or $\mu)$ in the event, with each lepton having $p_{T}^{l}>30 \mathrm{GeV}$ and $|\eta|<2.5$. The total number of events of the signal and background at the $14 \mathrm{TeV}$ and $27 \mathrm{TeV}$ run of LHC with $L=3000 \mathrm{fb}^{-1}$ are obtained after the optimization cut $\mathbb{E}_{T}>0.107 \mathrm{TeV}$. The $p p \rightarrow j j$ (where jets fake as leptons) background becomes negligibly small for the optimization cut $\mathscr{E}_{T}>0.107 \mathrm{TeV}$ along with $\left|M_{j j}-M_{Z}\right|<15 \mathrm{GeV}$. The signal events always remain $\sim 0$ for BP1 as $\operatorname{BR}\left(Z_{R} \rightarrow \tilde{\chi}_{1}^{0} \tilde{\chi}_{2}^{0}\right) \sim 0$.

\begin{tabular}{|c|c|c|c|c|c|c|c|c|}
\hline \multirow[b]{3}{*}{ Energy [TeV] } & \multicolumn{8}{|c|}{$Z+\mathbb{E}_{T}, Z \rightarrow l l$ final state through $\tilde{\chi}_{2}^{0}$} \\
\hline & \multicolumn{4}{|c|}{ SM backgrounds } & \multicolumn{2}{|c|}{ Total signal events } & \multicolumn{2}{|c|}{ Significance } \\
\hline & $Z Z$ & $W Z$ & $W W$ & $t \bar{t}$ & BP1 & BP2 & BP1 & $\mathrm{BP} 2$ \\
\hline 14 & 0.0 & 0.02 & 0 & 0 & $\cdots$ & 0.01 & $\cdots$ & $\cdots$ \\
\hline 27 & 1.39 & 1.59 & 0 & 0 & $\cdots$ & 7.26 & $\cdots$ & 4.2 \\
\hline
\end{tabular}


neutral gauge boson. Hence these cascade decay channels may lead to discovery or exclusion of these heavy gauge bosons through mono- $X(X=W, Z)$ plus $\mathscr{E}_{T}$ final state searches in the context of the HL-LHC and HE-LHC.

Similar to the previous cases, only the $Z \rightarrow l l,(l=e, \mu)$ decay modes have been considered. However the $Z \rightarrow \tau \tau$ can also enhance the signal as taus can give two SFOS leptons by decaying into electrons or muons. Using the same selection and optimization cuts of $\left|M_{l l}-M_{Z}\right|<$ $15 \mathrm{GeV}$ and $\mathscr{E}_{T}>0.107 \mathrm{TeV}$, it is found that the number of events at the $14 \mathrm{TeV}$ run of the LHC with luminosity $L=3000 \mathrm{fb}^{-1}$ remains the same for BP2, whereas the number of events increases by 0.1 at $27 \mathrm{TeV}$ LHC. Hence it is clear that the enhancement remains relatively small even if the tau leptons in the final state are considered.

The SFOS lepton pair final state can also come through the cascade decay process with $p p \rightarrow Z_{R} \rightarrow t \bar{t}, t \rightarrow W b$, $W \rightarrow l \nu$. It can be seen from the BPs that the branching of the heavy neutral gauge boson into the pair of top quark final state is quite large (see Table VII). As was already discussed in the mono- $W$ plus $\mathbb{E}_{T}$ searches, the isolation criteria of leptons significantly wane the number of events arising from top quark decays at the detector level. Furthermore, a b-jet veto completely removes any events in the final state and hence the enhancement in this channel remains negligible.

The process $p p \rightarrow Z_{R} \rightarrow \tilde{\chi}_{1}^{+} \tilde{\chi}_{1}^{-}$with $\tilde{\chi}_{1}^{ \pm} \rightarrow W \tilde{\chi}_{1}^{0}$ and $W \rightarrow l \nu$ can also provide SFOS lepton pair plus missing transverse energy in the final state. The branching of the heavy neutral gauge boson into a pair of charginos $\left(\tilde{\chi}_{1}^{ \pm}\right)$is around $24 \%$ for BP1 and 2\% for BP2 (see Table VII). The main discriminating variables important in this search are the transverse mass $M_{T}^{l_{i}}$ of the leptons and the missing transverse energy $\mathbb{E}_{T}$. For our analysis we choose the same selection cuts for the signal and background $V V$, $t \bar{t}(V=W, Z)$ as discussed before. Further we impose $200<M_{T}^{l_{1}}<3000 \mathrm{GeV}, M_{T}^{l_{2}}>200 \mathrm{GeV}$ and $\mathbb{E}_{T}>600 \mathrm{GeV}$ optimization cuts to maximize the signal significance. It is found that the number of events at the $27 \mathrm{TeV}$ run of the LHC with luminosity $L=3000 \mathrm{fb}^{-1}$ becomes less than 1 for $\mathrm{BP} 1$, whereas the number of events goes to 1.22 for $\mathrm{BP} 2$ and the total background attains a values of $\sim 90$. We find the corresponding significance 0.129 for BP2. It is to be noted that the number of signal events remains negligible at $14 \mathrm{TeV}$ LHC due to the small production cross section.

\section{SUMMARY AND CONCLUSIONS}

In this work, we have performed a detailed study of the heavy gauge boson decays and corresponding collider phenomenology in a minimal left-right supersymmetric model with automatic R-parity conservation. In our chosen scenario, the LR symmetry is broken in the SUSY limit, making the additional $W_{R}$ and $Z_{R}$ gauge bosons heavier than the SUSY particles. The heavy gauge bosons can thus decay into these SUSY states. We have studied the possible decay modes of the $W_{R}$ and $Z_{R}$ bosons into lighter electroweakinos and sfermions. In our initial analysis, the sfermions were kept heavier than the right-handed gauge bosons so as to prevent their decay into sfermion final states. Our results show that the heavy gauge bosons decaying into electroweakinos are strongly dependent on the composition of these states. We have thus considered all possibilities where the lightest neutralino (also the LSP in our model) is almost entirely composed of only a single type of gaugino or Higgsino state. The decay width of the heavy gauge bosons into these lighter electroweakinos becomes significant for the cases where the neutralino is either composed of the right-handed wino or is mostly composed of the Higgsino superpartner of bidoublet or triplet scalar. We then looked at the cases where the LSP can be a mixture of various Higgsino and gaugino states. Again, significantly large branching ratios for the heavy gauge boson decays were obtained for light neutralino states composed of a mixture of right-handed winos and Higgsinos. Allowing the sfermions to be lighter than these gauge bosons opened up new decay channels with the sfermions in the final state. The BR for these channels though remained quite small except for the $Z_{R}$ decays into down-type squarks. These extra decay channels can suppress the heavy gauge boson direct decays into SM particles and can potentially change the measured experimental bounds on their masses. Additionally, these decays can also suggest new collider signatures hinting towards the existence of the supersymmetric LR model.

We have performed detailed collider analysis of several SUSY and non-SUSY decays of the heavy gauge bosons in the context of HL-LHC and HE-LHC with c.m. energy of 14 and $27 \mathrm{TeV}$, respectively. We have analyzed the familiar dilepton and dijet channels arising from the direct decays of the $W_{R}$ and $Z_{R}$ bosons. We have also studied their onestep SUSY cascade decays into mono- $W+\mathbb{E}_{T}$ and mono$Z+\mathbb{E}_{T}$ final states. These signals have already been probed experimentally in the context of dark matter searches but not been considered as possible search channels for the heavy gauge bosons. To explore this possibility, we study the mono- $X+\mathbb{E}_{T}(X=W, Z)$ final states where the $X$ particles decay leptonically. The leptonic final states produce relatively clean signals that are easy to identify in a hadron-rich environment like the LHC experiment. We have chosen two benchmark points-BP1, which is more suited for the mono$W+\mathbb{E}_{T}$ search, and BP2 for mono- $Z+\mathbb{E}_{T}$ analysis. We have further optimized the selection cuts in order to enhance the signal significance over the SM backgrounds.

Our study shows that the dilepton final state gives promising results for the discovery of the heavy $Z_{R}$ boson at the HE-LHC while the dijet channel is better suited to search for $W_{R}$ bosons. Even if a heavy gauge boson is seen in these channels, the supersymmetric nature of the model still remains hidden. The mono- $X+\mathbb{E}_{T}$ channels, in conjunction with the dilepton and dijet channels, will not only 
be able to tell us about the existence of these heavy gauge bosons, but it can also provide significant hints towards the existence of SUSY particles.

\section{ACKNOWLEDGMENTS}

The work of B. B. was supported by the Department of Science and Technology, Government of India under Grant
No. IFA13-PH-75 (INSPIRE Faculty Award). The work of N.K. was supported by the Department of Science and Technology, Government of INDIA under SERB Grant No. PDF/2017/00372. The work of A.P. was partially supported by the Department of Science and Technology, Government of INDIA under SERB Grant No. PDF/2016/ 000202. The authors thank Sudhir K. Vempati, Rahool K. Barman, and Amit Adhikary for useful discussions.

\section{APPENDIX A: $W_{R}$ BOSON INTERACTION}

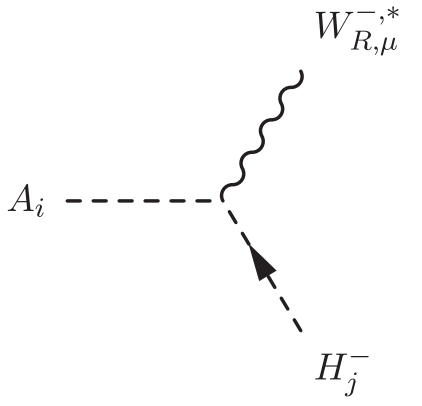

$$
\begin{aligned}
& \frac{g_{R}}{2}\left(-\sqrt{2} U_{j 1}^{H m, *} Z_{i 1}^{A}+\sqrt{2} U_{j 2}^{H m, *} Z_{i 2}^{A}\right. \\
& \left.+U_{j 4}^{H m, *} Z_{i 3}^{A}+U_{j 3}^{H m, *} Z_{i 4}^{A}\right)\left(-p_{\mu}^{H_{j}^{-}}+p_{\mu}^{A_{i}}\right)
\end{aligned}
$$

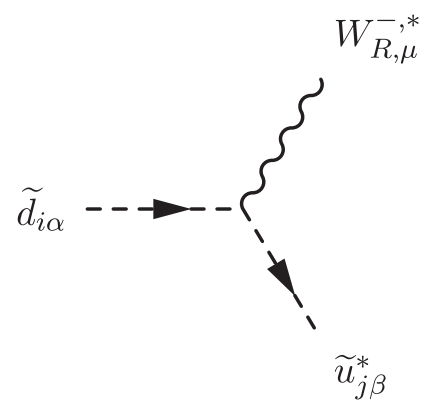

$$
\frac{i g_{R}}{\sqrt{2}} \delta_{\alpha \beta} \sum_{a=1}^{3} U_{i 3+a}^{D L, *} U_{j 3+a}^{U L}\left(p_{\mu}^{\widetilde{u}_{j \beta}^{*}}-p_{\mu}^{\widetilde{d}_{i \alpha}}\right)
$$

$$
\begin{aligned}
& W_{R, \mu}^{-, *} \\
& \tilde{e}_{i}-\ldots \int_{\widetilde{\nu}_{j}^{*}} \\
& \frac{i g_{R}}{\sqrt{2}} \sum_{a=1}^{3} U_{i 3+a}^{E L, *} U_{j 3+a}^{V L}\left(p_{\mu}^{\widetilde{\nu}_{j}^{*}}-p_{\mu}^{\widetilde{e}_{i}}\right)
\end{aligned}
$$



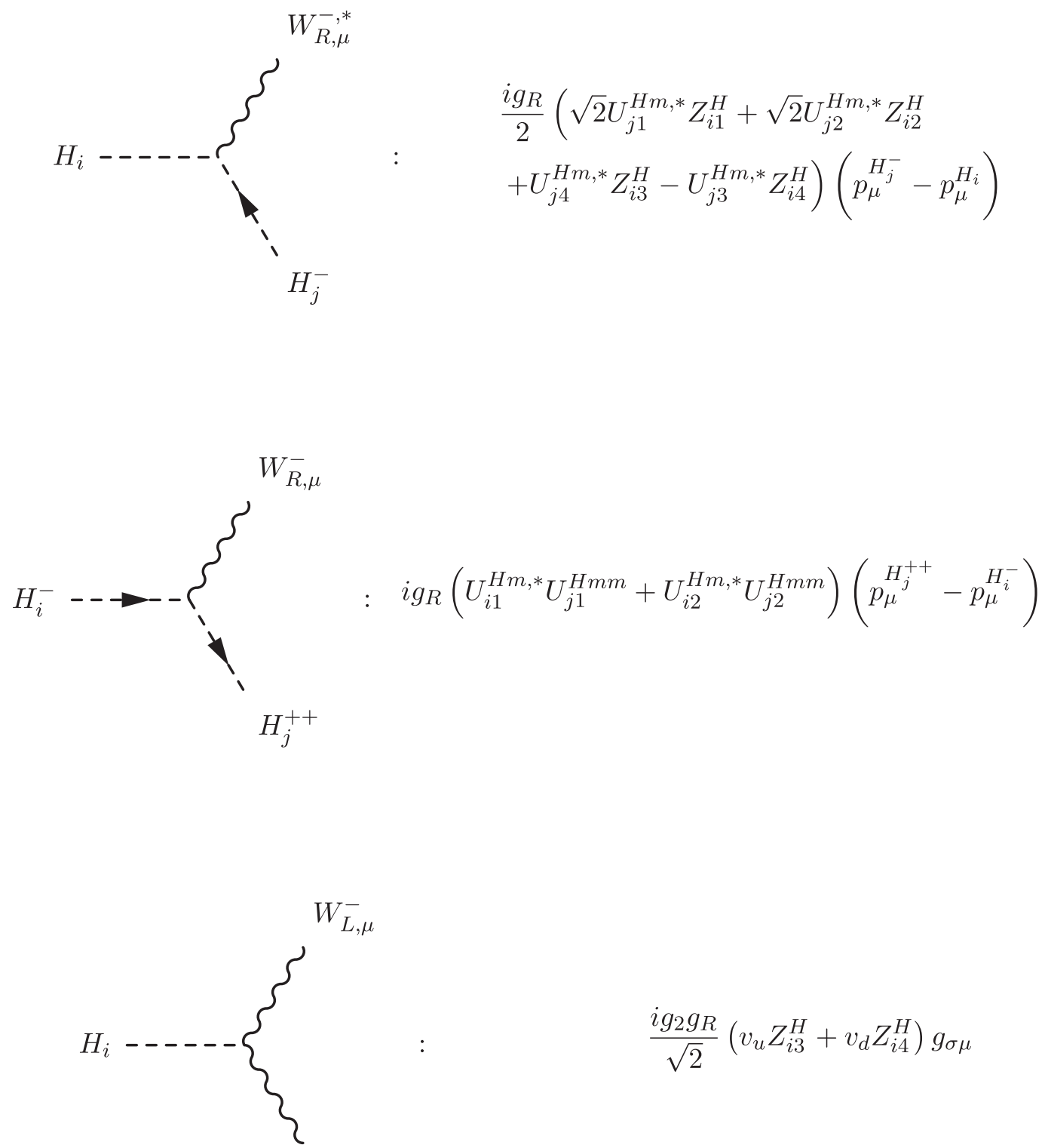

$$
\frac{i g_{2} g_{R}}{\sqrt{2}}\left(v_{u} Z_{i 3}^{H}+v_{d} Z_{i 4}^{H}\right) g_{\sigma \mu}
$$

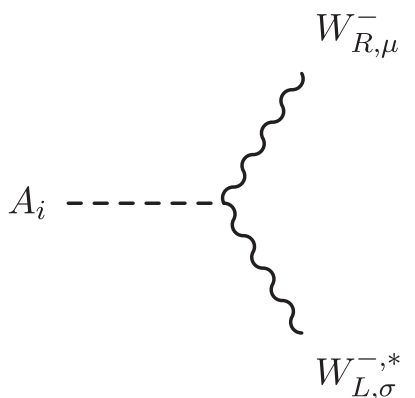

$$
\frac{g_{2} g_{R}}{\sqrt{2}}\left(v_{u} Z_{i 3}^{A}-v_{d} Z_{i 4}^{A}\right) g_{\sigma \mu}
$$




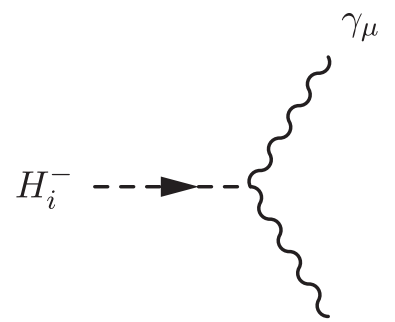

$$
\begin{aligned}
& i g_{R}\left\{\left(2 g_{V} Z_{11}^{Z}-g_{R} Z_{31}^{Z}\right)\left(\bar{v}_{R} U_{i 1}^{H m, *}+v_{R} U_{i 2}^{H m, *}\right)\right. \\
& \left.+\frac{g_{2}}{\sqrt{2}} Z_{21}^{Z}\left(v_{d} U_{i 4}^{H m, *}-v_{u} U_{i 3}^{H m, *}\right)\right\} g_{\sigma \mu}
\end{aligned}
$$

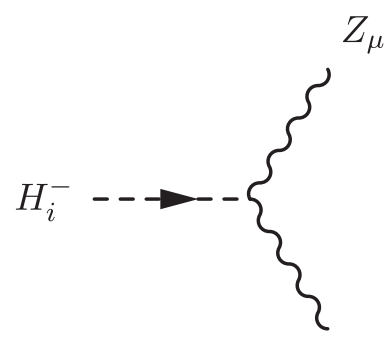

$$
\begin{aligned}
& i g_{R}\left\{\left(2 g_{V} Z_{12}^{Z}-g_{R} Z_{32}^{Z}\right)\left(\bar{v}_{R} U_{i 1}^{H m, *}+v_{R} U_{i 2}^{H m, *}\right)\right. \\
& \left.+\frac{g_{2}}{\sqrt{2}} Z_{22}^{Z}\left(v_{d} U_{i 4}^{H m, *}-v_{u} U_{i 3}^{H m, *}\right)\right\} g_{\sigma \mu}
\end{aligned}
$$

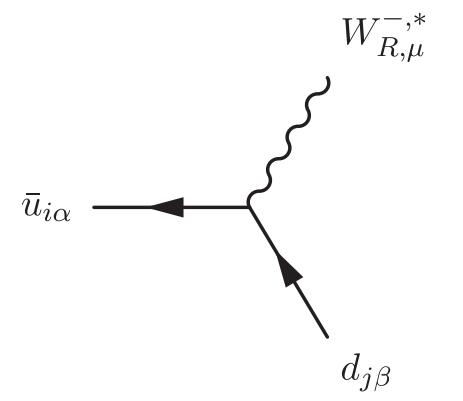

$$
-\frac{i g_{R}}{\sqrt{2}} \delta_{\alpha \beta} \sum_{a=1}^{3} U_{i a}^{U u, *} U_{j a}^{U d} \gamma_{\mu} P_{R}
$$

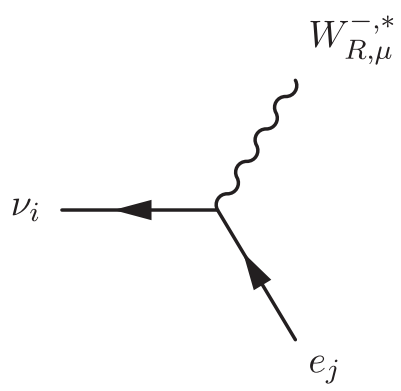

$$
-\frac{i g_{R}}{\sqrt{2}} \sum_{a=1}^{3} U_{i 3+a}^{P M N S, *} U_{j a}^{U e} \gamma_{\mu} P_{R}
$$



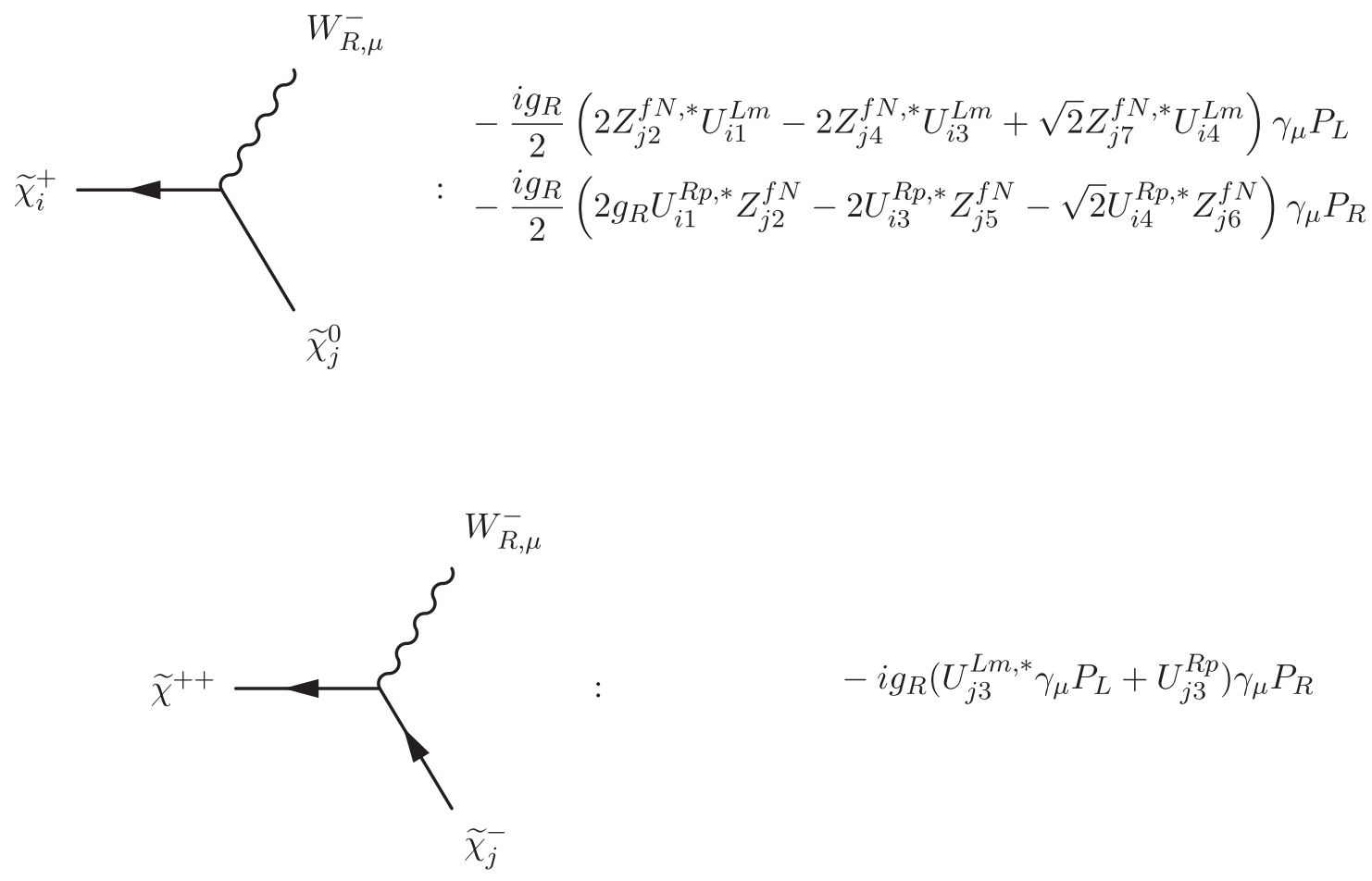

\section{APPENDIX B: $Z_{R}$ BOSON INTERACTION}
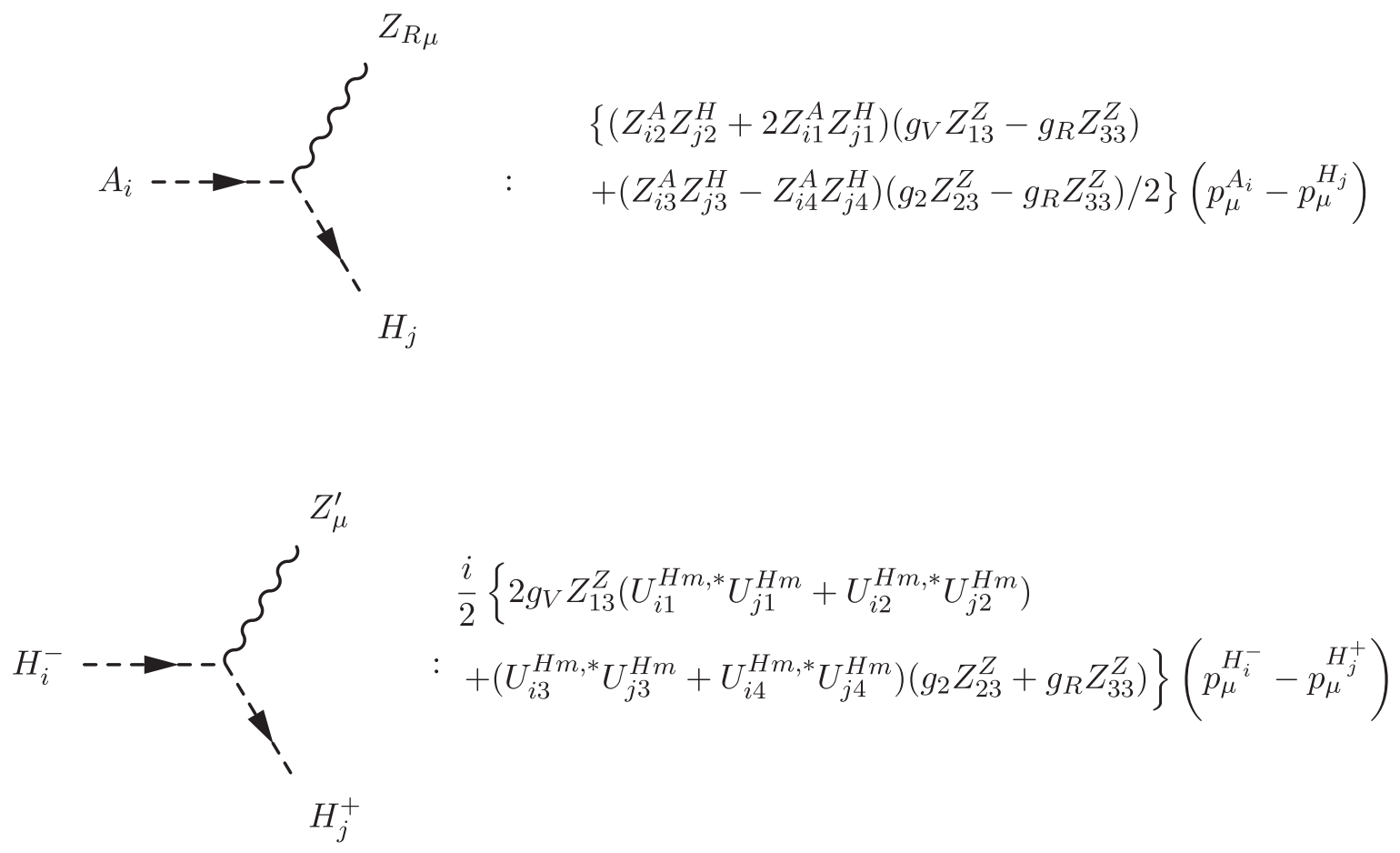


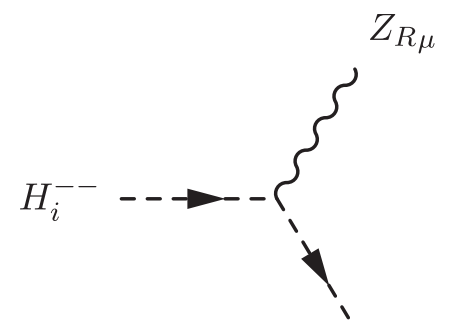

$$
\begin{aligned}
& i\left(U_{i 1}^{H m m, *} U_{j 1}^{H m m}+U_{i 2}^{H m m, *} U_{j 2}^{H m m}\right)\left(g_{V} Z_{13}^{Z}+g_{R} Z_{33}^{Z}\right) \\
& \times\left(p_{\mu}^{H_{i}^{--}}-p_{\mu}^{H_{j}^{++}}\right)
\end{aligned}
$$

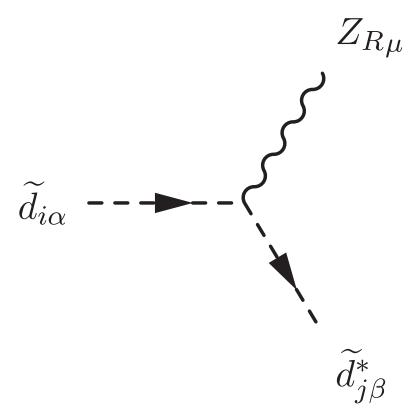

$$
\begin{aligned}
& -\frac{i}{6} \delta_{\alpha \beta}\left\{\sum_{a=4}^{3} U_{i a}^{D L, *} U_{j a}^{D L}\left(-3 g_{R} Z_{33}^{Z}+g_{V} Z_{13}^{Z}\right)\right. \\
& \left.+\sum_{a=1}^{3} U_{i a}^{D L, *} U_{j a}^{D L}\left(-3 g_{2} Z_{23}^{Z}+g_{V} Z_{13}^{Z}\right)\right\}\left(p_{\mu}^{\widetilde{d}_{i \alpha}}-{p_{\mu}}_{j \beta}^{*}\right)
\end{aligned}
$$

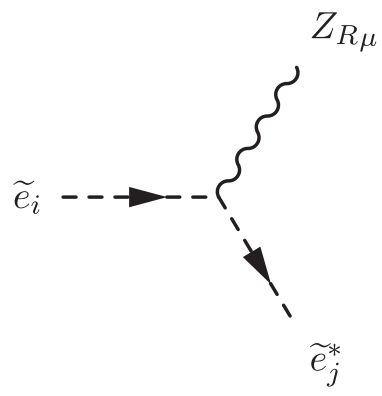

$$
\begin{aligned}
& \frac{i}{2}\left\{\sum_{a=4}^{3} U_{i a}^{E L, *} U_{j a}^{E L}\left(g_{V} Z_{13}^{Z}+g_{R} Z_{33}^{Z}\right)\right. \\
& \left.+\sum_{a=1}^{3} U_{i a}^{E L, *} U_{j a}^{E L}\left(g_{2} Z_{23}^{Z}+g_{V} Z_{13}^{Z}\right)\right\}\left(p_{\mu}^{\widetilde{e}_{i}}-p_{\mu}^{\widetilde{e}_{j}^{*}}\right)
\end{aligned}
$$

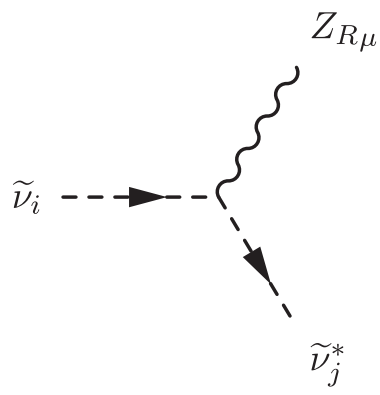

$$
\begin{aligned}
& \frac{i}{2}\left\{\sum_{a=1}^{3} U_{i 3+a}^{V L} U_{j 3+a}^{V L}\left(g_{V} Z_{13}^{Z}-g_{R} Z_{33}^{Z}\right)\right. \\
& \left.+\sum_{a=1}^{3} U_{i a}^{V L} U_{j a}^{V L}\left(-g_{2} Z_{23}^{Z}+g_{V} Z_{13}^{Z}\right)\right\}\left(p_{\mu}^{\widetilde{\nu}_{i}}-p_{\mu}^{\widetilde{\nu}_{j}^{*}}\right)
\end{aligned}
$$



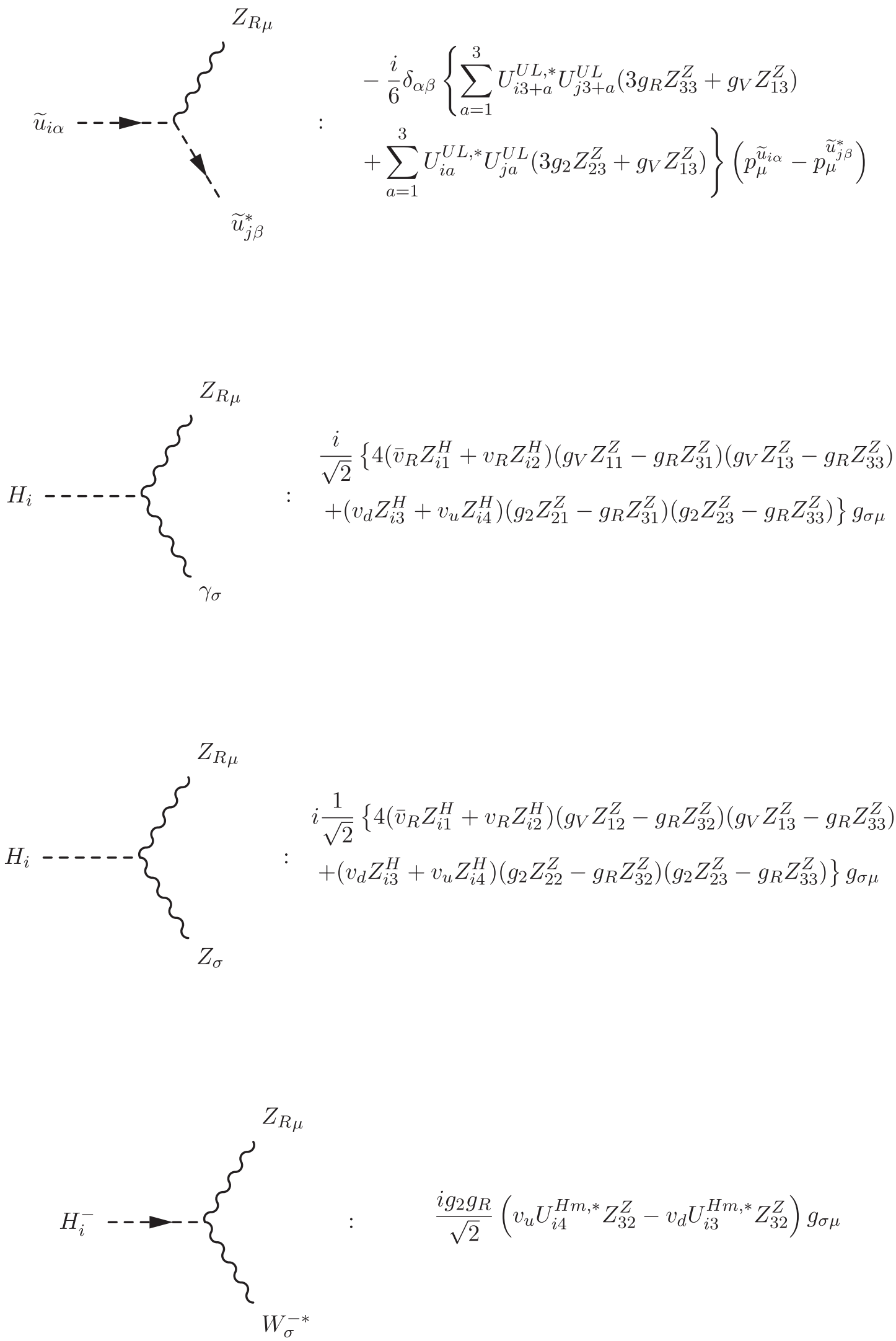

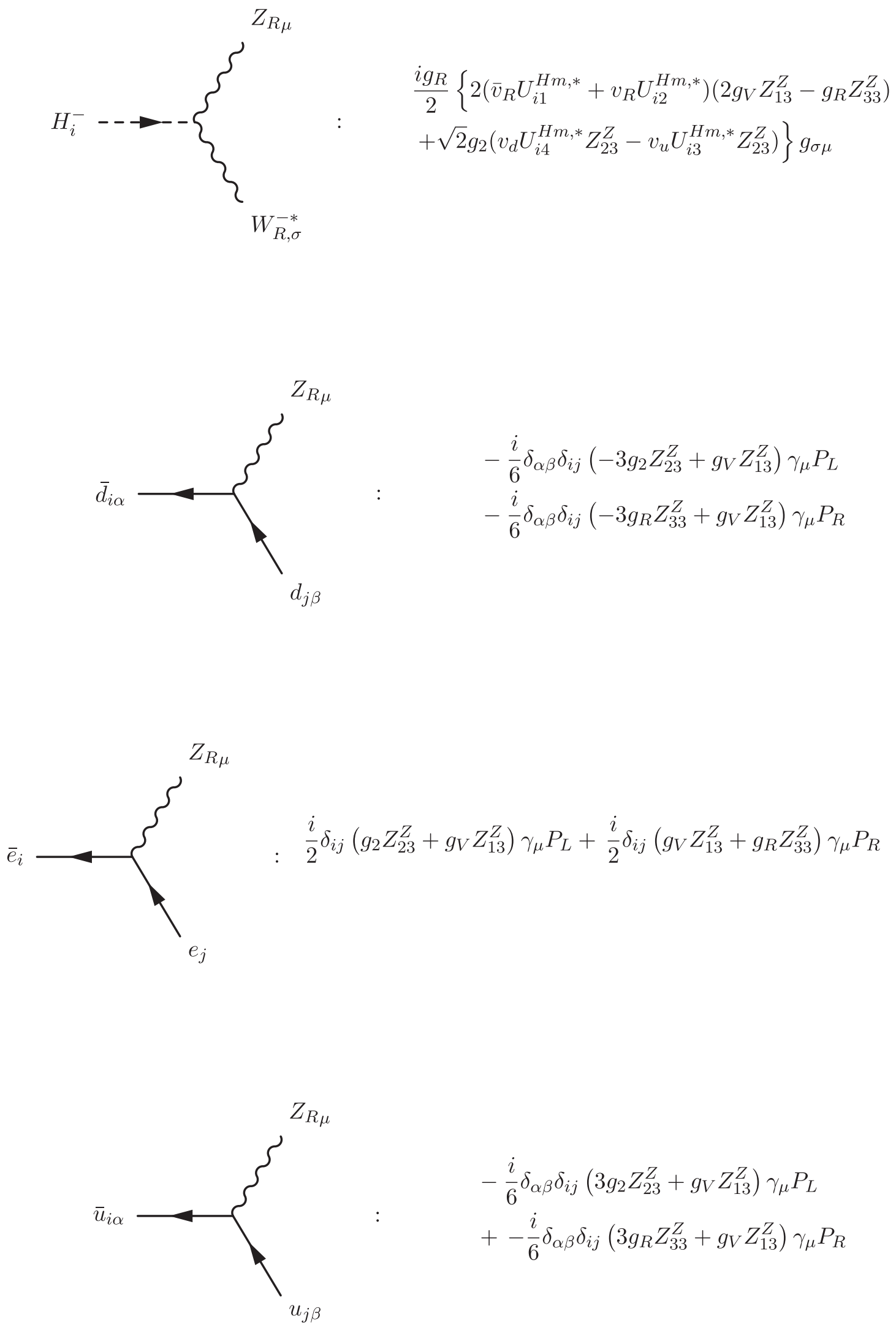

$$
\begin{aligned}
& -\frac{i}{6} \delta_{\alpha \beta} \delta_{i j}\left(3 g_{2} Z_{23}^{Z}+g_{V} Z_{13}^{Z}\right) \gamma_{\mu} P_{L} \\
& +-\frac{i}{6} \delta_{\alpha \beta} \delta_{i j}\left(3 g_{R} Z_{33}^{Z}+g_{V} Z_{13}^{Z}\right) \gamma_{\mu} P_{R}
\end{aligned}
$$



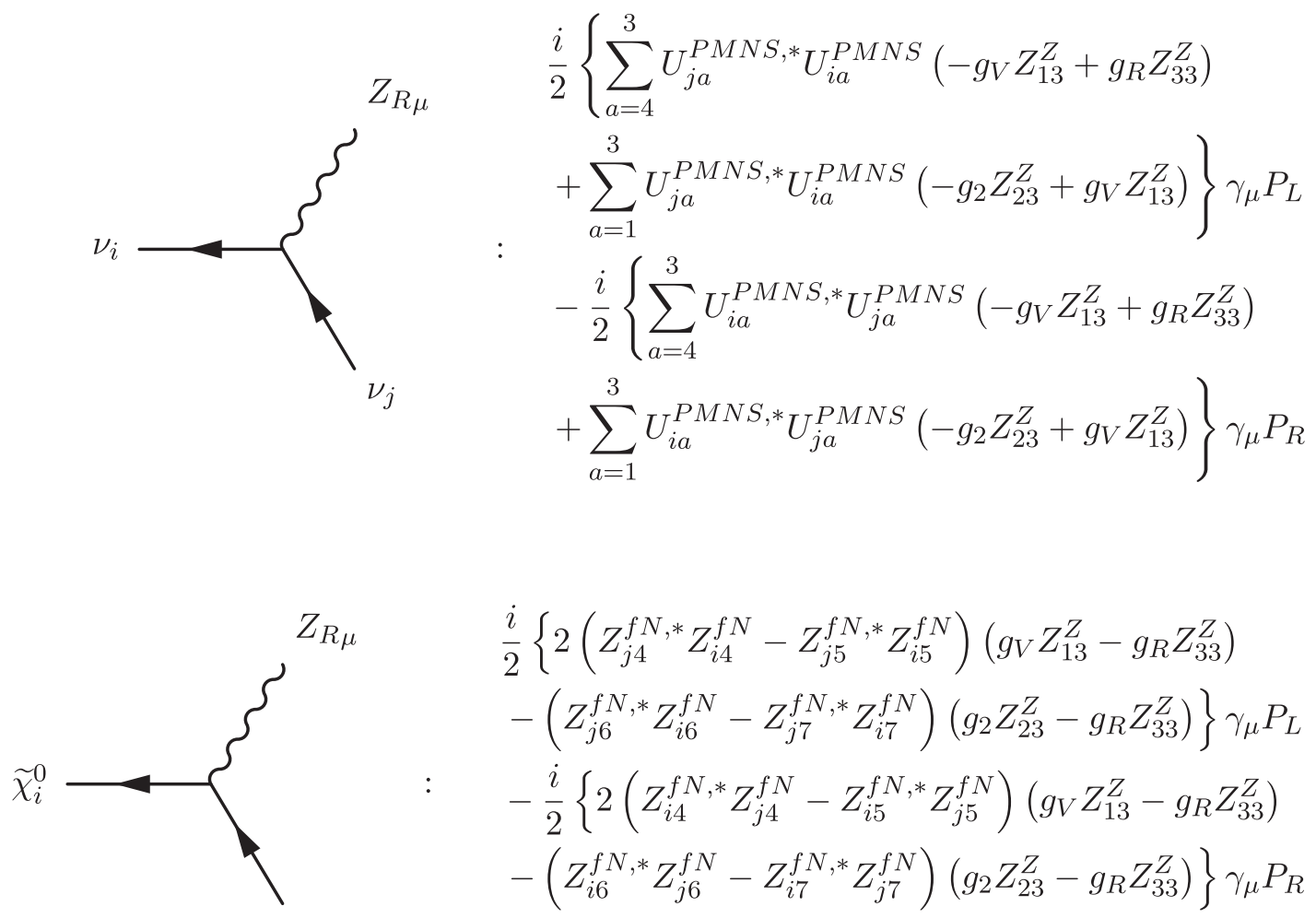

$$
\begin{aligned}
& \frac{i}{2}\left\{2\left(Z_{j 4}^{f N, *} Z_{i 4}^{f N}-Z_{j 5}^{f N, *} Z_{i 5}^{f N}\right)\left(g_{V} Z_{13}^{Z}-g_{R} Z_{33}^{Z}\right)\right. \\
& \left.-\left(Z_{j 6}^{f N, *} Z_{i 6}^{f N}-Z_{j 7}^{f N, *} Z_{i 7}^{f N}\right)\left(g_{2} Z_{23}^{Z}-g_{R} Z_{33}^{Z}\right)\right\} \gamma_{\mu} P_{L} \\
& -\frac{i}{2}\left\{2\left(Z_{i 4}^{f N, *} Z_{j 4}^{f N}-Z_{i 5}^{f N, *} Z_{j 5}^{f N}\right)\left(g_{V} Z_{13}^{Z}-g_{R} Z_{33}^{Z}\right)\right. \\
& \left.-\left(Z_{i 6}^{f N, *} Z_{j 6}^{f N}-Z_{i 7}^{f N, *} Z_{j 7}^{f N}\right)\left(g_{2} Z_{23}^{Z}-g_{R} Z_{33}^{Z}\right)\right\} \gamma_{\mu} P_{R}
\end{aligned}
$$
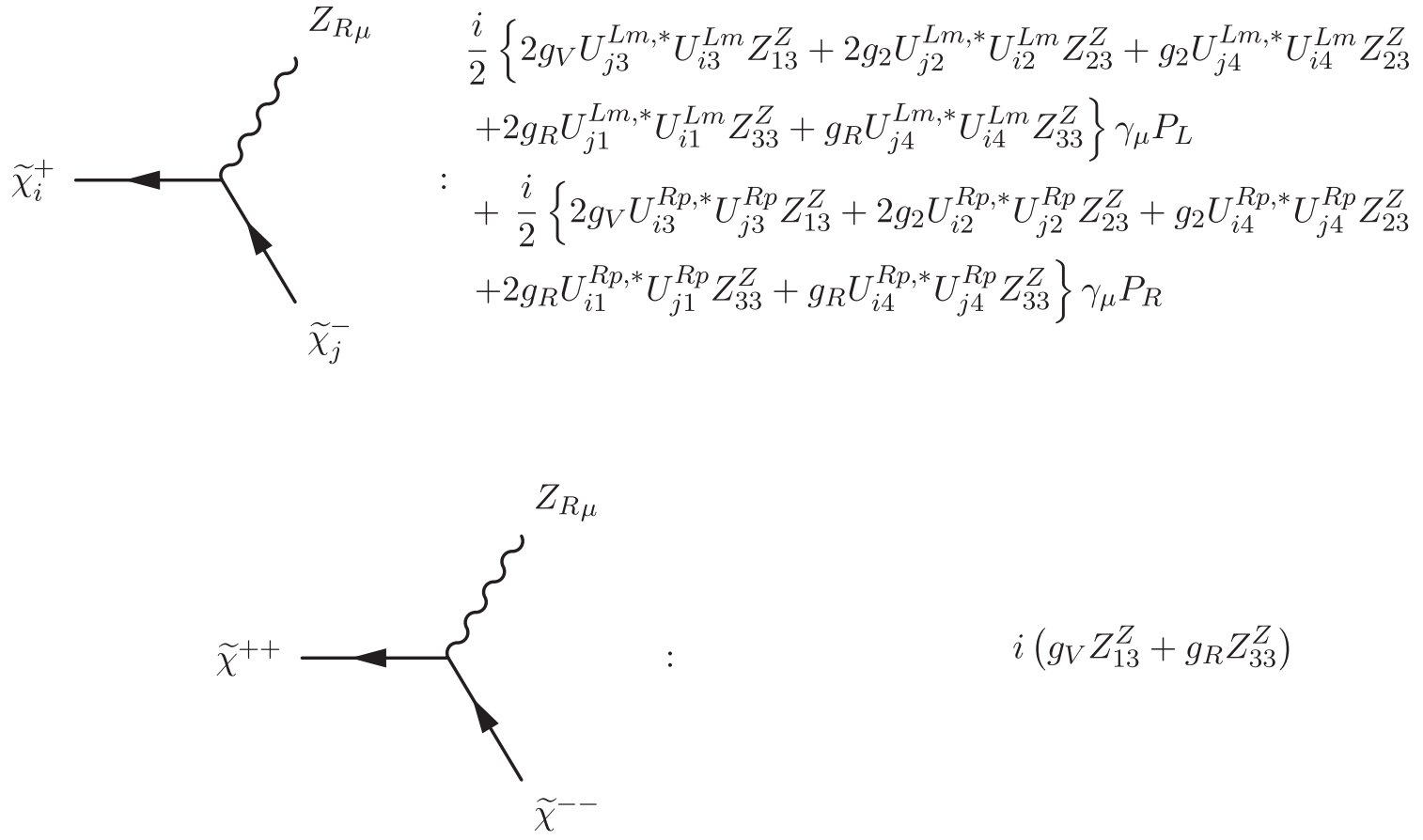

$$
i\left(g_{V} Z_{13}^{Z}+g_{R} Z_{33}^{Z}\right)
$$




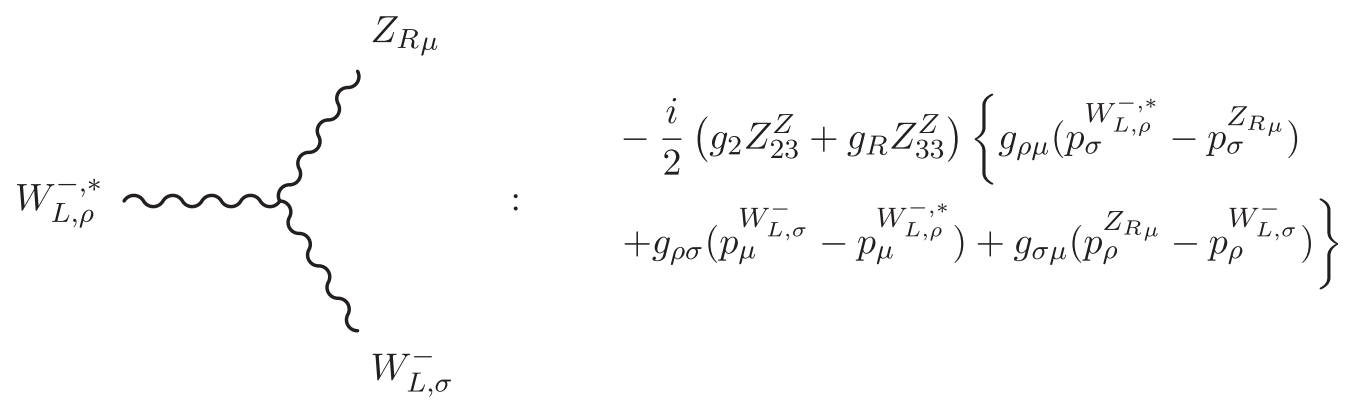

[1] S. Chatrchyan et al. (CMS Collaboration), Phys. Lett. B 716, 30 (2012); 716, 1 (2012).

[2] C. S. Aulakh, A. Melfo, A. Rasin, and G. Senjanovic, Phys. Rev. D 58, 115007 (1998); C. S. Aulakh, A. Melfo, and G. Senjanovic, Phys. Rev. D 57, 4174 (1998); C. S. Aulakh, K. Benakli, and G. Senjanovic, Phys. Rev. Lett. 79, 2188 (1997); Z. Chacko and R. N. Mohapatra, Phys. Rev. D 58, 015003 (1998); M. Frank and B. Korutlu, Phys. Rev. D 83, 073007 (2011).

[3] R. N. Mohapatra and J. C. Pati, Phys. Rev. D 11, 566 (1975); G. Senjanovic and R. N. Mohapatra, Phys. Rev. D 12, 1502 (1975).

[4] M. A. B. Beg and H.-S. Tsao, Phys. Rev. Lett. 41, 278 (1978); R. N. Mohapatra and G. Senjanovic, Phys. Lett. B 79, 283 (1978); K. S. Babu and R. N. Mohapatra, Phys. Rev. D 41, 1286 (1990); S. M. Barr, D. Chang, and G. Senjanovic, Phys. Rev. Lett. 67, 2765 (1991); R. N. Mohapatra and A. Rasin, Phys. Rev. Lett. 76, 3490 (1996); R. Kuchimanchi, Phys. Rev. Lett. 76, 3486 (1996); R. N. Mohapatra, A. Rasin, and G. Senjanovic, Phys. Rev. Lett. 79, 4744 (1997); K. S. Babu, B. Dutta, and R. N. Mohapatra, Phys. Rev. D 65, 016005 (2001); R. Kuchimanchi, Phys. Rev. D 82, 116008 (2010).

[5] R. D. Peccei and H. R. Quinn, Phys. Rev. Lett. 38, 1440 (1977).

[6] P. Minkowski, Phys. Lett. B 67, 421 (1977); T. Yanagida, Conf. Proc. C 7902131, 95 (1979).S. Glashow, in Quarks and Leptons, Cargèse 1979, edited by M. Lévy et al. (Plenum, New York, 1980); M. Gell-Mann, P. Ramond, and R. Slansky, Proceedings of the Supergravity Stony Brook Workshop, New York, 1979, edited by P. Van Niewenhuizen and D. Freeman (North-Holland, Amsterdam, 1979); R. Mohapatra and G. Senjanović, Phys. Rev. Lett. 44, 912 (1980).

[7] K. S. Babu, X.-G. He, and E. Ma, Phys. Rev. D 36, 878 (1987); E. Ma, Phys. Rev. D 36, 274 (1987); R. Kuchimanchi and R. N. Mohapatra, Phys. Rev. D 48, 4352 (1993); R. Kuchimanchi and R. N. Mohapatra, Phys. Rev. Lett. 75, 3989 (1995); K. Huitu, P. N. Pandita, and K. Puolamaki, Phys. Lett. B 423, 97 (1998); Y. Zhang, H. An,
X.-d. Ji, and R. N. Mohapatra, Phys. Rev. D 78, 011302 (2008); A. Patra and S. K. Rai, Phys. Rev. D 98, 015033 (2018).

[8] K. S. Babu and A. Patra, Phys. Rev. D 93, 055030 (2016).

[9] K. S. Babu and R. N. Mohapatra, Phys. Lett. B 668, 404 (2008).

[10] A. M. Sirunyan et al. (CMS Collaboration), J. High Energy Phys. 06 (2018) 120.

[11] G. Aad et al. (ATLAS Collaboration), Phys. Lett. B 796, 68 (2019).

[12] M. Aaboud et al. (ATLAS Collaboration), J. High Energy Phys. 10 (2017) 182.

[13] M. Aaboud et al. (ATLAS Collaboration), Phys. Rev. Lett. 120, 161802 (2018).

[14] A. M. Sirunyan et al. (CMS Collaboration), Phys. Lett. B 792, 107 (2019).

[15] A. M. Sirunyan et al. (CMS Collaboration), J. High Energy Phys. 06 (2018) 128.

[16] V. Khachatryan et al. (CMS Collaboration), Phys. Lett. B 770, 278 (2017).

[17] M. Aaboud et al. (ATLAS Collaboration), Phys. Rev. D 96, 052004 (2017).

[18] A. M. Sirunyan et al. (CMS Collaboration), J. High Energy Phys. 08 (2017) 029.

[19] T. Gherghetta, T. A. Kaeding, and G. L. Kane, Phys. Rev. D 57, 3178 (1998).

[20] J. Kang and P. Langacker, Phys. Rev. D 71, 035014 (2005).

[21] M. Baumgart, T. Hartman, C. Kilic, and L. T. Wang, J. High Energy Phys. 11 (2007) 084.

[22] G. Corcella and S. Gentile, Nucl. Phys. B866, 293 (2013); B868, 554(E) (2013).

[23] G. Corcella, Eur. Phys. J. C 75, 264 (2015).

[24] J. Y. Araz, G. Corcella, M. Frank, and B. Fuks, J. High Energy Phys. 02 (2018) 092.

[25] M. E. Krauss, B. O'Leary, W. Porod, and F. Staub, Phys. Rev. D 86, 055017 (2012).

[26] L. Basso, B. Fuks, M. E. Krauss, and W. Porod, J. High Energy Phys. 07 (2015) 147. 
[27] M. Dhuria, C. Hati, R. Rangarajan, and U. Sarkar, J. Cosmol. Astropart. Phys. 09 (2015) 035.

[28] M.E. Krauss and W. Porod, Phys. Rev. D 92, 055019 (2015).

[29] K. S. Babu, B. Dutta, and R. N. Mohapatra, Phys. Rev. D 60, 095004 (1999).

[30] M. Aaboud et al. (ATLAS Collaboration), Phys. Rev. D 97, 112001 (2018).

[31] M. Aaboud et al. (ATLAS Collaboration), Eur. Phys. J. C 78, 625 (2018).

[32] G. Degrassi, S. Heinemeyer, W. Hollik, P. Slavich, and G. Weiglein, Eur. Phys. J. C 28, 133 (2003); B. C. Allanach, A. Djouadi, J. L. Kneur, W. Porod, and P. Slavich, J. High Energy Phys. 09 (2004) 044; Phys. Rev. D 75, 055005 (2007); Phys. Rev. Lett. 100, 191602 (2008); 101, 039901 (2008); S. Heinemeyer, O. Stal, and G. Weiglein, Phys. Lett. B 710, 201 (2012); A. Arbey, M. Battaglia, A. Djouadi, and F. Mahmoudi, J. High Energy Phys. 09 (2012) 107.

[33] K. S. Babu, A. Patra, and S. K. Rai, Phys. Rev. D 88, 055006 (2013).

[34] T. Kobayashi and O. Vives, Phys. Lett. B 506, 323 (2001).

[35] J. A. Casas, A. Lleyda, and C. Munoz, Nucl. Phys. B471, 3 (1996).

[36] D. Chowdhury, R. M. Godbole, K. A. Mohan, and S. K. Vempati, J. High Energy Phys. 02 (2014) 110; 03 (2018) 149.
[37] M. Frank, B. Fuks, K. Huitu, S. K. Rai, and H. Waltari, J. High Energy Phys. 05 (2017) 015.

[38] A. Chatterjee, M. Frank, B. Fuks, K. Huitu, S. Mondal, S. K. Rai, and H. Waltari, Phys. Rev. D 99, 035017 (2019).

[39] F. Staub, Comput. Phys. Commun. 184, 1792 (2013).

[40] F. Staub, Adv. High Energy Phys. 2015, 840780 (2015).

[41] W. Porod, Comput. Phys. Commun. 153, 275 (2003).

[42] W. Porod and F. Staub, Comput. Phys. Commun. 183, 2458 (2012).

[43] J. Alwall, R. Frederix, S. Frixione, V. Hirschi, F. Maltoni, O. Mattelaer, H.-S. Shao, T. Stelzer, P. Torrielli, and M. Zaro, J. High Energy Phys. 07 (2014) 079.

[44] T. Sjostrand, S. Mrenna, and P. Z. Skands, J. High Energy Phys. 05 (2006) 026.

[45] J. de Favereau, C. Delaere, P. Demin, A. Giammanco, V. Lemaître, A. Mertens, and M. Selvaggi (DELPHES 3 Collaboration), J. High Energy Phys. 02 (2014) 057.

[46] R. K. Barman, B. Bhattacherjee, A. Chakraborty, and A. Choudhury, Phys. Rev. D 94, 075013 (2016).

[47] ATLAS Collaboration, CERN Report No. ATLAS-CONF2014-017, 2015.

[48] G. Aad et al. (ATLAS Collaboration), Phys. Rev. D 90, 012004 (2014); M. Aaboud et al. (ATLAS Collaboration), Eur. Phys. J. C 78, 625 (2018).

[49] V. Khachatryan et al. (CMS Collaboration), J. High Energy Phys. 04 (2015) 025. 